\title{
A DETAILED ANALYSIS OF THE DUST FORMATION ZONE OF IRC +10216 DERIVED FROM MID-INFRARED BANDS OF $\mathrm{C}_{2} \mathrm{H}_{2}$ AND HCN
}

\author{
J. P. Fonfría and J. Cernicharo \\ Departamento Astrofísica Molecular e Infrarroja, Instituto de la Estructura de la Materia (IEM), Consejo Superior de Investigaciones \\ Científicas (CSIC), 28006 Madrid, Spain; jpablo.fonfria@damir.iem.csic.es, cerni@damir.iem.csic.es \\ M. J. RICHTER ${ }^{1}$ \\ Physics Department, University of California, Davis, CA 95616; richter@physics.ucdavis.edu \\ AND \\ J. H. LACY ${ }^{1}$ \\ Astronomy Department, University of Texas, Austin, TX 78712; lacy@shrub.as.utexas.edu \\ Received 2007 February 27; accepted 2007 September 19
}

\begin{abstract}
A spectral survey of IRC +10216 has been carried out in the range 11-14 $\mu \mathrm{m}$ with a spectral resolution of about $4 \mathrm{~km} \mathrm{~s}^{-1}$. We have identified a forest of lines in six bands of $\mathrm{C}_{2} \mathrm{H}_{2}$ involving the vibrational states from the ground to $3 \nu_{5}$ and in two bands of $\mathrm{HCN}$, involving the vibrational states from the ground up to $2 \nu_{2}$. Some of these transitions are observed also in $\mathrm{H}^{13} \mathrm{CCH}$ and $\mathrm{H}^{13} \mathrm{CN}$. We have estimated the kinetic, vibrational, and rotational temperatures and the abundances and column densities of $\mathrm{C}_{2} \mathrm{H}_{2}$ and $\mathrm{HCN}$ between $1 R_{*}$ and $300 R_{*}\left(\simeq 1.5 \times 10^{16} \mathrm{~cm}\right)$ by fitting about 300 of these rovibrational lines. The envelope can be divided into three regions with approximate boundaries at $0.019^{\prime \prime}$ (the stellar photosphere), $0.1^{\prime \prime}$ (the inner dust formation zone), and $0.4^{\prime \prime}$ (outer dust formation zone). Most of the lines might require a large microturbulence broadening. The derived abundances of $\mathrm{C}_{2} \mathrm{H}_{2}$ and $\mathrm{HCN}$ increase by factors of 10 and 4 , respectively, from the innermost envelope outward. The derived column densities for both $\mathrm{C}_{2} \mathrm{H}_{2}$ and $\mathrm{HCN}$ are $\simeq 1.6 \times$ $10^{19} \mathrm{~cm}^{-2}$. Vibrational states up to $3000 \mathrm{~K}$ above ground are populated, suggesting pumping by near-infrared radiation from the star and innermost envelope. Low rotational levels can be considered under LTE, while those with $J>20-30$ are not thermalized. A few lines require special analysis to deal with effects like overlap with lines of other molecules.
\end{abstract}

Subject headings: line: identification - line: profiles - stars: AGB and post-AGB - stars: carbon stars: individual (IRC +10216) - surveys

Online material: color figures, machine-readable tables

\section{INTRODUCTION}

IRC +10216 is a carbon asymptotic giant branch (AGB) star surrounded by a circumstellar envelope (CSE) of gas and dust. Since it is the nearest AGB star of this type and the strongest infrared object in the sky, it has become the paradigm for this kind of source (see $\S 3.1$ for more information about previous work). The central star itself cannot be observed directly due to the large dust optical depth, but the observational data from the CSE suggest that it is a physically and chemically rich environment. Although this source has been studied many times since its discovery by Neugebauer \& Leighton (1969), most of these physical and chemical processes remain poorly understood across the envelope (see, for example, Agúndez \& Cernicharo 2006).

The physical conditions in the innermost part of the CSE maintain chemical thermodynamical equilibrium. However, the gas could depart from the latter due to the action of periodic shock waves arising from stellar pulsation (Cherchneff et al. 1992; Agúndez \& Cernicharo 2006). At the temperatures prevailing in these zones, most atoms are integrated into molecular species, primarily $\mathrm{H}_{2}$. The chemistry is so rich that more than $50 \%$ of the molecules known in space were first discovered in this source (see, for example, Morris et al. 1975; Betz 1981; Cernicharo et al.

\footnotetext{
${ }^{1}$ Visiting Astronomer at the Infrared Telescope Facility, which is operated by the University of Hawaii under contract from the National Aeronautics and Space Administration.
}

2000, 2004 and references therein). Some of these molecules, such as $\mathrm{SiO}$ (Schöier et al. 2006), SiS, CS (Lucas et al. 1995), and metal-bearing species (Cernicharo \& Guélin 1987; Cernicharo et al. 2000), are refractory and are formed in the inner envelope. As soon as the temperature of the gas is below a critical value, these species start to condense and form dust grains. Other species, mainly radicals, display emission from the external shell of the envelope ( $A_{V} \simeq 1$ mag; Dayal \& Bieging 1993, 1995; Lucas et al. 1995; Cernicharo \& Guélin 1996; Lindqvist et al. 2000), where the Galactic UV field starts to photodissociate the stable molecules formed in the inner CSE and neutral-radical reactions produce long carbon chain radicals (Cernicharo \& Guélin 1996; Guélin et al. 1997) and also the interesting gas-phase oxygen chemistry (Agúndez \& Cernicharo 2006).

The most abundant molecular species, after $\mathrm{H}_{2}$, are $\mathrm{CO}, \mathrm{C}_{2} \mathrm{H}_{2}$, and HCN. CO has an abundance of $\simeq 8 \times 10^{-4}$ from $1 R_{*}$ to beyond $1000 R_{*}$ (Lafont et al. 1982; Knapp \& Morris 1985; Agúndez \& Cernicharo 2006). $\mathrm{C}_{2} \mathrm{H}_{2}$ is predicted by thermodynamical chemical equilibrium models (Tejero \& Cernicharo 1991) to be the most abundant molecule after $\mathrm{CO}$ and $\mathrm{H}_{2}$. Its abundance has been derived to be $\simeq 8 \times 10^{-5}$ in the zone $1 R_{*}-40 R_{*}$ from infrared observations (Keady \& Ridgway 1993; Cernicharo et al. 1999). The abundance of HCN, $\simeq(3-4) \times 10^{-5}$, has been derived from midinfrared (MIR), far-infrared (FIR), and radio observations (Keady \& Ridgway 1993; Cernicharo et al. 1996, 1999).

Radiation pressure by stellar photons accelerates the dust grains formed near the star. For the densities prevailing in these 
dusty regions of the CSE, the coupling between gas and dust is high and hence the gas is also accelerated (Gilman 1972; Kwok 1975). Keady et al. (1988) have found that the terminal velocity of the gas is about $14 \mathrm{~km} \mathrm{~s}^{-1}$ (see also Cernicharo et al. 2000) and is reached before $20 R_{*}$. These authors also found that the velocity field seems to have more than one acceleration regime occurring at different places of the inner CSE. These zones might be related to the condensation temperature of different refractory molecular species.

The main goal of this work is to learn more about the physical conditions and chemical composition of the CSE of IRC +10216 from modeling the line profiles of $\mathrm{C}_{2} \mathrm{H}_{2}$ and $\mathrm{HCN}$ observed in the MIR with the high-resolution Texas Echelon Cross Echelle Spectrograph (TEXES; Lacy et al. 2002). These observations are presented in $\S 2$. The models we have used to fit the observational data are described in $\S 3$ and Appendices A and B, where we discuss the line identification and give the dipole moment for the different observed vibrational transitions. The continuum emission is analyzed in $\S 4$. The results concerning $\mathrm{C}_{2} \mathrm{H}_{2}$ and its isotopologs are presented in $\S \S 5$ and 6 , and those related to $\mathrm{HCN}$ and $\mathrm{H}^{13} \mathrm{CN}$ in $\S 7$. The results obtained for $\mathrm{C}_{2} \mathrm{H}_{2}$ and $\mathrm{HCN}$, their uncertainties, and the sensitivity of the model to the physical conditions of the CSE are discussed and analyzed in $\S 8$. Finally, the results are summarized in $\S 9$.

\section{OBSERVATIONS}

We observed IRC +10216 with TEXES (Lacy et al. 2002) at the NASA Infrared Telescope Facility on 2002 December 12 (UT), corresponding to an IR stellar phase $\phi_{\mathrm{IR}} \simeq 0.08$ (following Monnier et al. 1998). We used the TEXES high-resolution echelon grating with a first-order grating as the cross-disperser. In this mode, we obtained a spectral coverage of roughly $0.25 \mu \mathrm{m}$ per setting. To cover the entire range shown here, $11.6-13.9 \mu \mathrm{m}$, we required 10 separate settings and stored the data in 10 different files.

At these wavelengths, the echelon orders are larger than our detector, meaning that there are gaps in the spectrum between orders. The gaps increase in size toward longer wavelengths. Telluric features become stronger and more frequent toward $13.9 \mu \mathrm{m}$. In regions where the telluric atmosphere was nearly opaque $(<5 \%$ transmission) we discarded the data, resulting in additional gaps.

When using the first-order grating as the cross-disperser, the TEXES slit must be very small to prevent orders from overlapping. For these observations the slit was $2.2^{\prime \prime}$ long. With the short slit, we nod IRC +10216 off the slit for sky subtraction. Sky conditions were good enough that we do not believe that this introduced any significant systematics.

The data were reduced with the standard TEXES pipeline (Lacy et al. 2002). The pipeline corrects optical distortions, combines nod pairs, removes spikes, flat-fields, performs correction for telluric absorption, establishes a frequency scale using telluric features, and extracts a spectrum. We normalized each spectrum before analysis using a fourth-degree polynomial to estimate the baseline.

Typically, observations of asteroids provide the best telluric corrections. However, IRC +10216 is so much brighter than the available asteroids that we would have limited our signal-tonoise ratio by using asteroid measurements. Instead, we used a blackbody-sky difference spectrum to correct for the atmosphere. In the ideal case of the atmosphere, telescope, and blackbody being at a uniform temperature, the difference spectrum indicates the absorption from the sky. In our experience, this procedure for atmospheric correction works fairly well except for telluric water vapor, which can change on a short timescale and from one line of sight to another.
TABLE 1

Observed Spectrum toward IRC +10216

\begin{tabular}{|c|c|c|c|}
\hline $\begin{array}{l}\text { Frequency } \\
\left(\mathrm{cm}^{-1}\right) \\
(1)\end{array}$ & $\begin{array}{l}\text { Normalized } \\
\text { Flux } \\
\text { (2) }\end{array}$ & $\begin{array}{c}\text { Atmospheric } \\
\text { Transmission } \\
\text { (3) }\end{array}$ & $\begin{array}{c}\text { Model } \\
\text { (4) }\end{array}$ \\
\hline $721.579 \ldots \ldots$ & 1.023 & 0.1810 & 1.000 \\
\hline 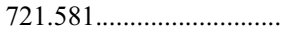 & 1.089 & 0.1877 & 1.000 \\
\hline $721.583 \ldots \ldots \ldots \ldots \ldots \ldots \ldots \ldots$ & 1.126 & 0.1940 & 1.000 \\
\hline 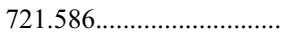 & 1.121 & 0.2009 & 1.000 \\
\hline 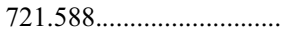 & 1.111 & 0.2068 & 1.000 \\
\hline 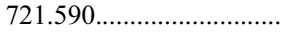 & 1.079 & 0.2127 & 1.000 \\
\hline 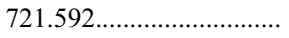 & 1.019 & 0.2166 & 1.000 \\
\hline 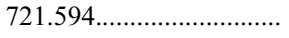 & 0.9778 & 0.2159 & 1.000 \\
\hline 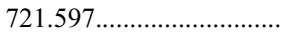 & 0.8599 & 0.2144 & 1.000 \\
\hline 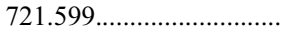 & 0.7520 & 0.2155 & 1.000 \\
\hline
\end{tabular}

Notes.-Observed spectrum toward IRC +10216 ranging from 721 to $864 \mathrm{~cm}^{-1}$. Col. (1) contains the observed frequencies in $\mathrm{cm}^{-1}$ corrected from velocity shifts due to proper motions of the source, frequency calibration, and uncertainties related to the band center of laboratory-determined frequencies. The observed flux, having removed the baseline, can be found in col. (2). Col. (3) accounts for an estimation of the atmospheric transmission during the observations at the considered frequencies. The model results have been included in col. (4) to allow comparisons. See the text for details. Table 1 is published in its entirety in the electronic edition of the Astrophysical Journal. A portion is shown here for guidance regarding its form and content.

The observed spectrum covers the wavenumber range $720-$ $864 \mathrm{~cm}^{-1}$. Taking into account the molecules detected in this source (Keady \& Ridgway 1993; Cernicharo et al. 2000), there are several candidates that could contribute to the observed features: $\mathrm{C}_{2} \mathrm{H}_{2}, \mathrm{HCN}$, and SiS, including their isotopologs. All of them have vibrational bands in the observed wavelength range (see Figs. 1-6). Although many lines of SiS and other molecules have been identified in the figures, they will be analyzed and studied in a forthcoming paper.

The spectra were corrected from the source movement by identifying and modeling most of the $\mathrm{C}_{2} \mathrm{H}_{2} \nu_{5} R_{e}$ and $\mathrm{HCN} \nu_{2} R_{e}$ lines in the spectrum and calculating the mean shift between the observed frequencies and those published from laboratory work (Rothman et al. 2003). This shift is $\simeq 45 \mathrm{~km} \mathrm{~s}^{-1}$. However, several files need an extra offset smaller than $2 \mathrm{~km} \mathrm{~s}^{-1}$ due to small uncertainties in the observational process. Moreover, we had to correct the central frequency of some $\mathrm{C}_{2} \mathrm{H}_{2}$ and $\mathrm{HCN}$ bands taken from the HITRAN Database ${ }^{2}$ by blueshifting them in less than $1.5 \mathrm{~km} \mathrm{~s}^{-1}$. A sample of the velocity-corrected data is shown in Table 1 .

In order to fit the continuum, we have used Infrared Space Observatory (ISO) SWS observations carried out on 1996 May 31 (UT) that correspond to $\phi_{\mathrm{IR}} \simeq 0.34$.

\section{THE MODEL}

\subsection{The Physical Structure of IRC +10216 from Previous Work}

IRC +10216 is ejecting matter (gas and dust) at a rate of (1-2) $\times 10^{-5} M_{\odot} \mathrm{yr}^{-1}$ (Keady et al. 1988; Cernicharo et al. 1996, 1999). The derived dust ejection rate is in the range $\simeq(2-4) \times$ $10^{-7} M_{\odot} \mathrm{yr}^{-1}$ (Ridgway \& Keady 1988; Men'shchikov et al. 2001). The star is pulsating with a period of $636 \pm 3$ days (Ridgway \& Keady 1988; Dyck et al. 1991; Jones et al. 1990). The stellar effective temperature, $T_{\text {eff }}$, quoted in the literature varies from author to author: $2330 \pm 350 \mathrm{~K}$ (Ridgway \& Keady 1988), $2200 \pm 150 \mathrm{~K}$ (Ivezić \& Elitzur 1996), $1915 \mathrm{~K}$ at phase

\footnotetext{
${ }^{2}$ See http://cfa-www.harvard.edu/hitran/.
} 

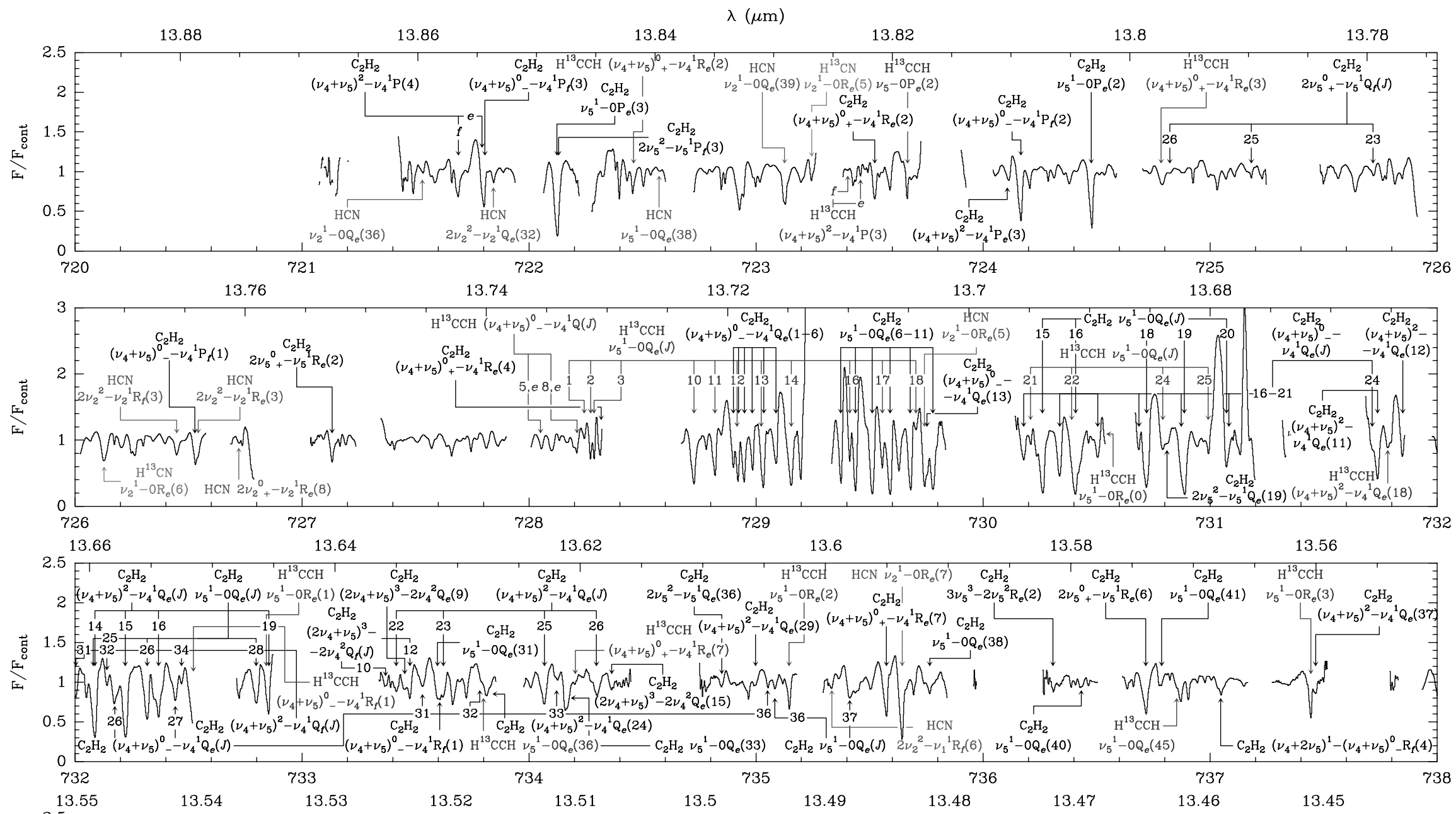

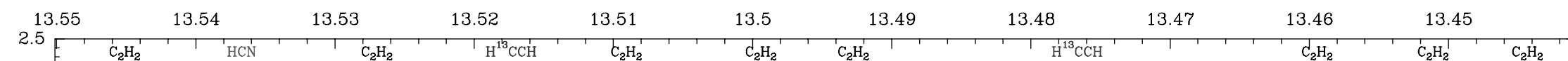

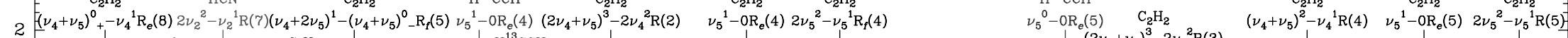

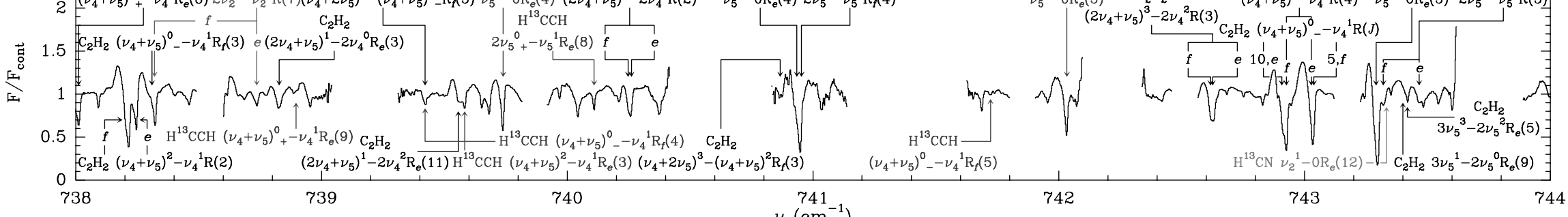

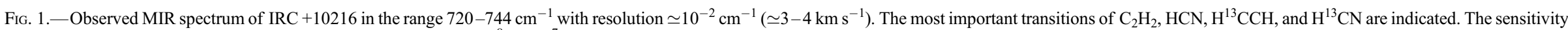

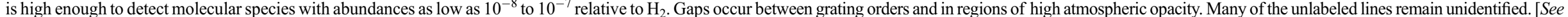
the electronic edition of the Journal for a color version of this figure.] 

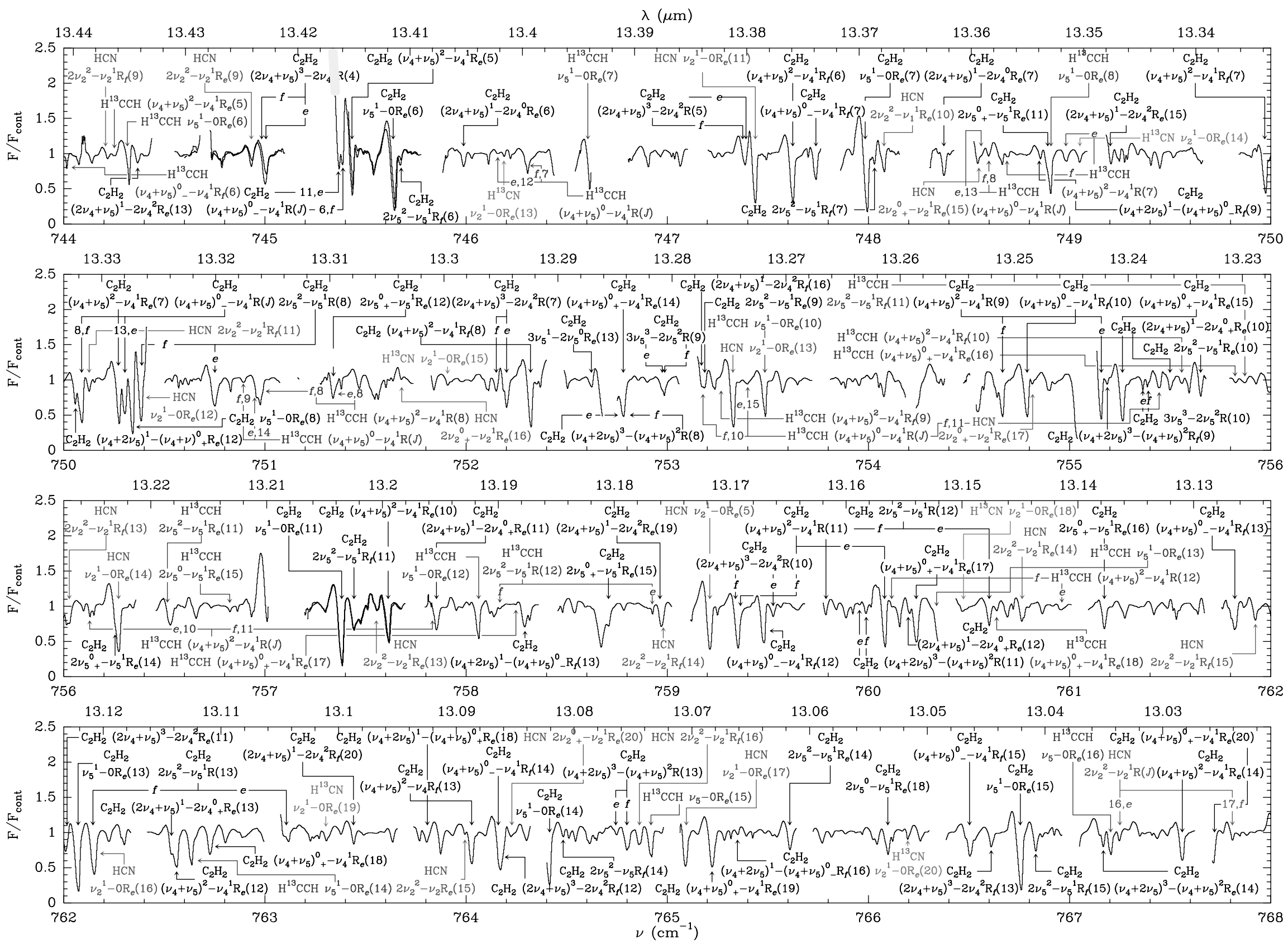

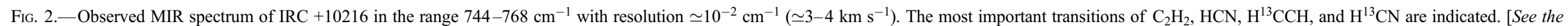
electronic edition of the Journal for a color version of this figure.] 


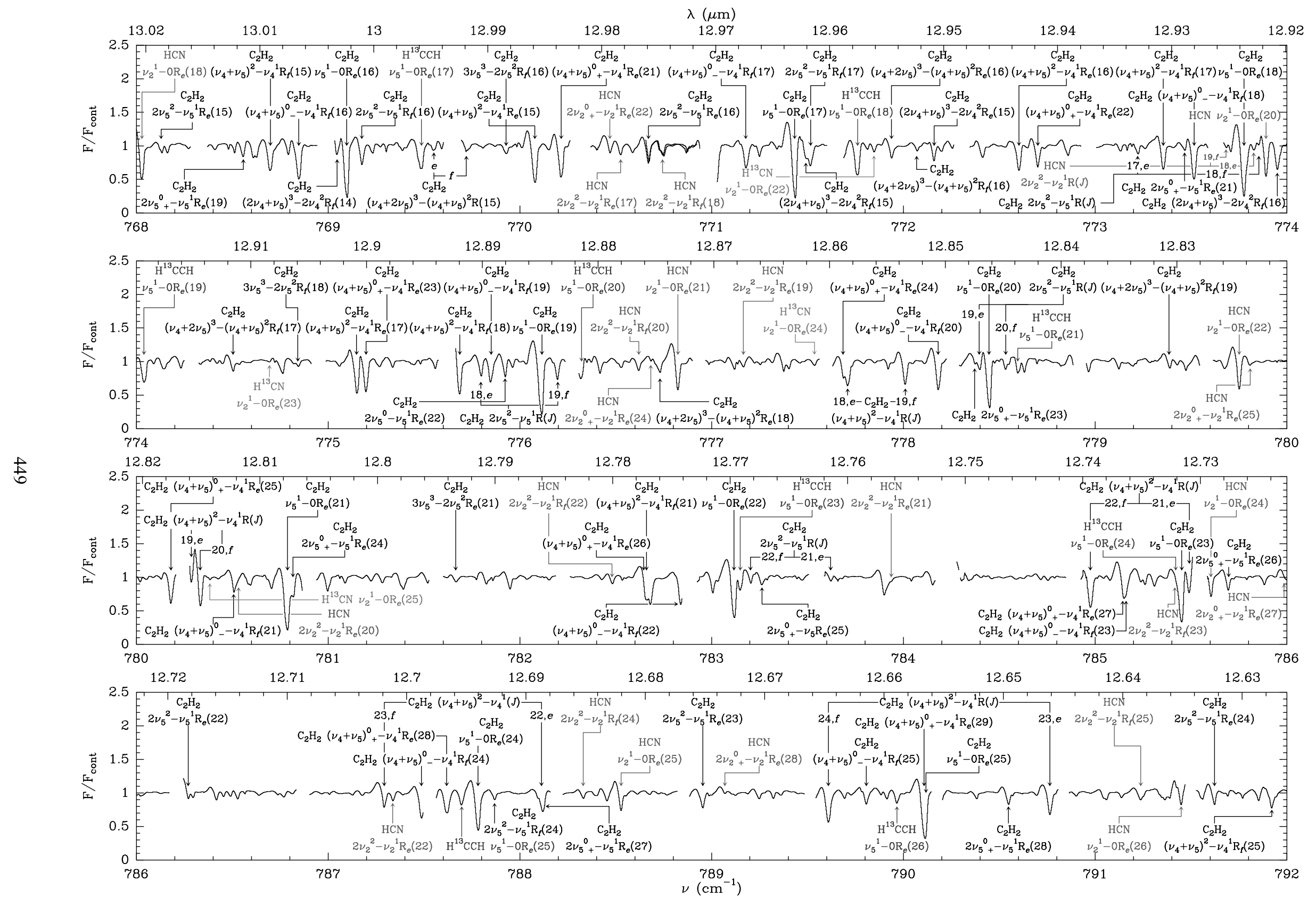

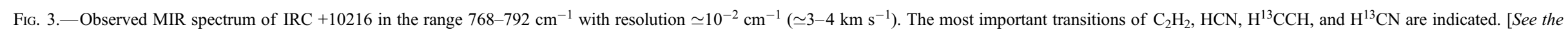
electronic edition of the Journal for a color version of this figure.] 


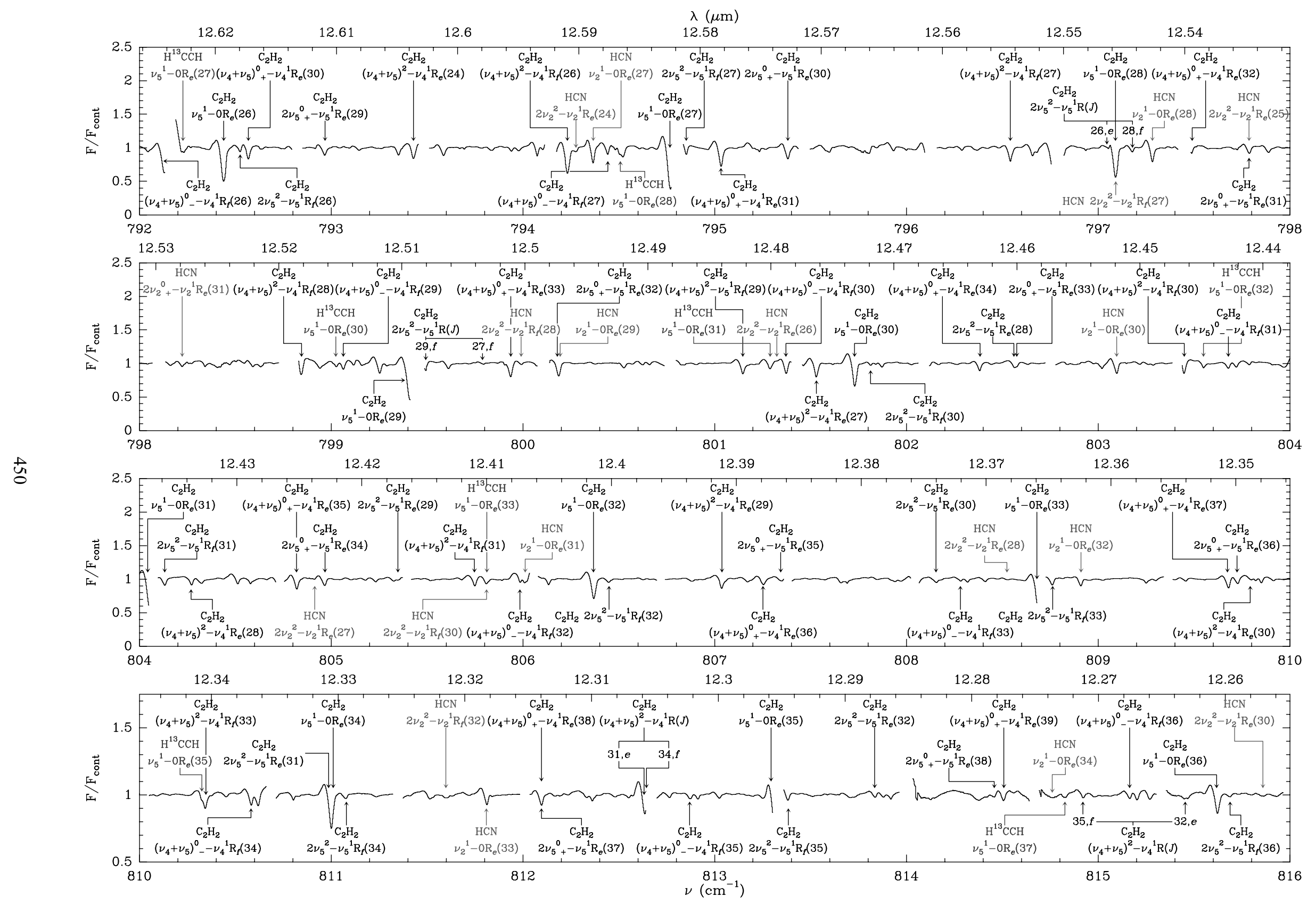

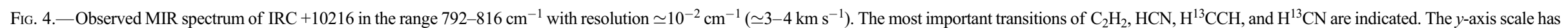
been changed beyond $810 \mathrm{~cm}^{-1}$ to show the molecular features more clearly. [See the electronic edition of the Journal for a color version of this figure.] 


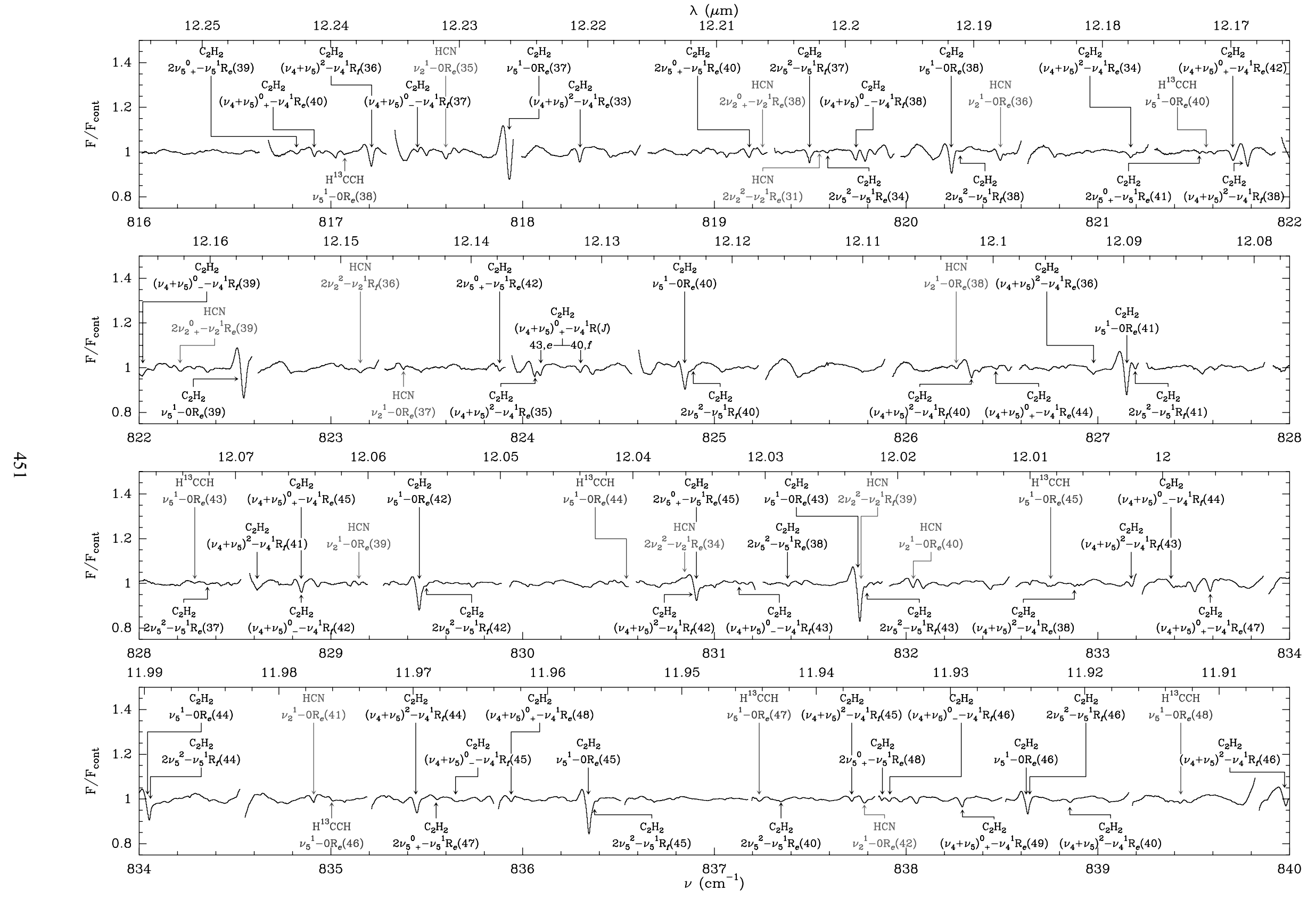

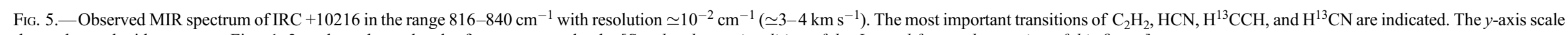
has been changed with respect to Figs. 1-3 to show the molecular features more clearly. [See the electronic edition of the Journal for a color version of this figure.] 

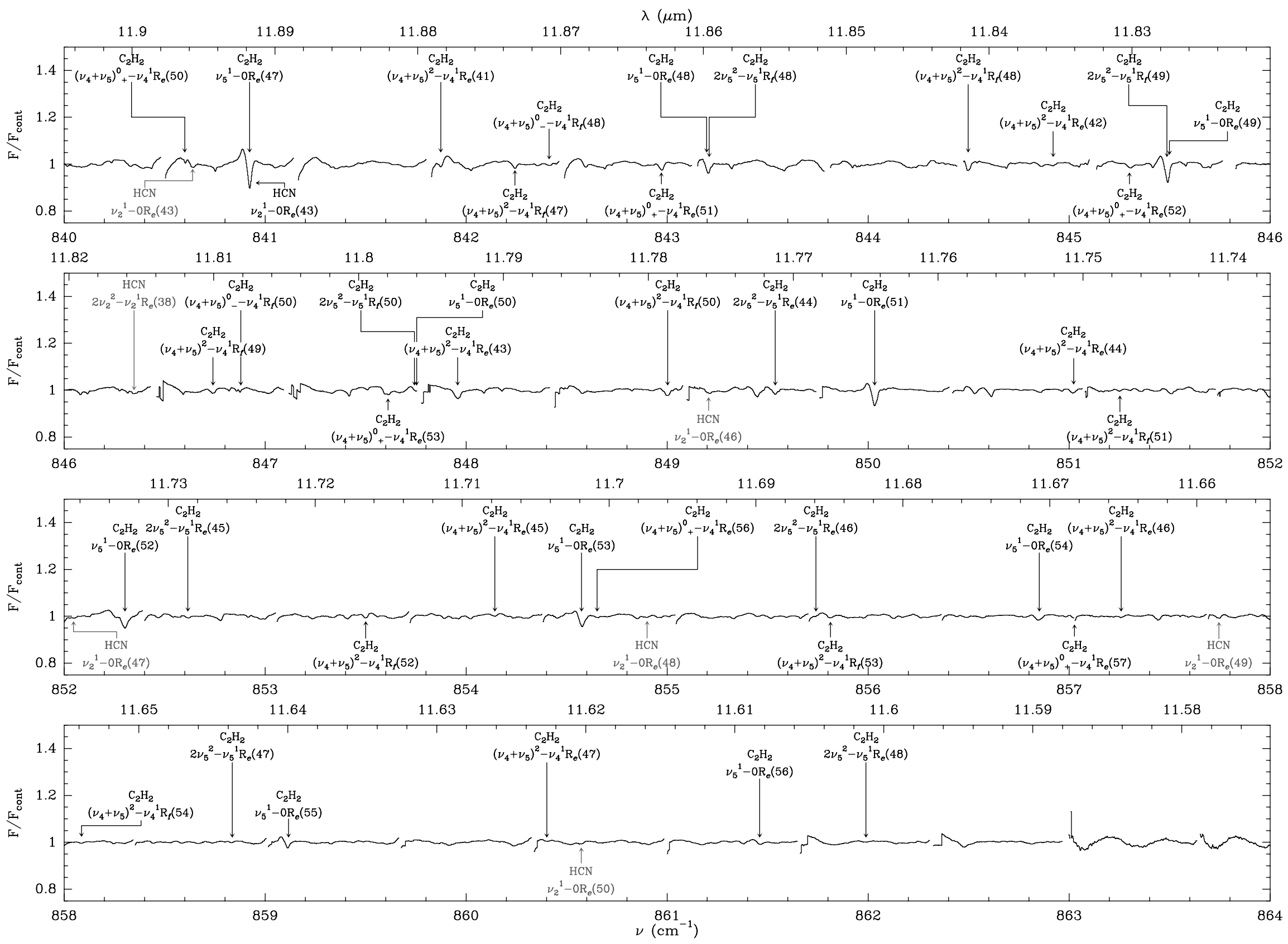

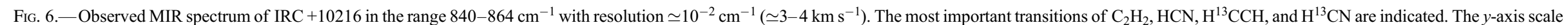
has been changed with respect to Figs. 1-3 to show the molecular features more clearly. [See the electronic edition of the Journal for a color version of this figure.] 
0.16 and $2105 \mathrm{~K}$ at phase 0.27 (Bergeat et al. 2001), and 2800 and $2500 \mathrm{~K}$ for maximum and minimum brightness, respectively (Men'shchikov et al. 2001).

The distance of IRC +10216 is poorly established; values vary between 120 and 300 pc (Doty \& Leung 1997; Keady et al. 1988; Weigelt et al. 2002; Bergeat et al. 2001; Loup et al. 1993; Cernicharo et al. 2000; Herbig \& Zappala 1970). From interferometric and lunar occultation data, Ridgway \& Keady (1988) have obtained a stellar angular radius of $0.019^{\prime \prime} \pm 0.003^{\prime \prime}$, Keady et al. (1988) assumed 0.023", the value derived by Monnier et al. (2000) is $0.022^{\prime \prime}$, and Men'shchikov et al. (2001) suggest an angular stellar radius ranging from $0.014^{\prime \prime}$ to $0.018^{\prime \prime}$ over the whole period of pulsation (corresponding to $\simeq 815 R_{\odot}$ for a distance of $200 \mathrm{pc}$ derived from observations at $1.65,2.2,3.15$, and $4.95 \mu \mathrm{m}$; $\simeq 970 R_{\odot}$ with a distance of $200 \mathrm{pc} ; \simeq 635 R_{\odot}$ for a distance of $135 \mathrm{pc}$ from $8-12 \mu \mathrm{m}$ observations; and a radius ranging from 390 to $500 R_{\odot}$ with an assumed distance of $130 \mathrm{pc}$, from measurements between $0.6 \mu \mathrm{m}$ and $6 \mathrm{~mm}$, respectively).

Infrared observations during a lunar occultation (Ridgway \& Keady 1988) showed that the dusty inner envelope of IRC +10216 is asymmetric. There is a large amount of dust in the equatorial plane and two bright lobes along the poles where the dust density and resulting extinction are lower. Interferometric observations by Weigelt et al. (1998) revealed at least four clumps in the lobes. Later observations showed that these clumps evolve with time (Weigelt et al. 2002; Tuthill et al. 2005) with timescales of $\simeq 1 \mathrm{yr}$ (Men'shchikov et al. 2002). The large-scale density profile of the dust reveals nearly concentric shells corresponding to increased ejection of matter over periods of 200-800 yr lasting 20-40 yr each (Mauron \& Huggins 1999, 2000; Murakawa et al. 2002). The latter ejection episodes are compatible with results by Men'shchikov et al. (2001) that suggest that the star has experienced at least two episodes of high mass loss over the last $1000 \mathrm{yr}$.

From the work of Keady et al. (1988) dust grains are formed by amorphous carbon (AC) with some inclusions of silicon carbide ( $\mathrm{SiC}$ ) and perhaps other components containing $\mathrm{Mg}$ or $\mathrm{S}$. $\mathrm{SiC}$ condenses in the photosphere, forming the seeds of dust grains (the condensation temperature of $\mathrm{SiC}$ is $\simeq 2000 \mathrm{~K}$; Men'shchikov et al. 2001). Ivezić \& Elitzur (1996) have calculated the relative amounts of the main components of the dust grains to be $\simeq 95 \%$ $\mathrm{AC}, 3 \%-8 \% \mathrm{SiC}$, and less than $10 \% \mathrm{MgS}$. Other authors have suggested that the molecular bands detected in the continuum can be produced by molecules more complex than $\mathrm{MgS}$ (e.g., Men'shchikov et al. 2001).

The gas is accelerated to $\simeq 2 \mathrm{~km} \mathrm{~s}^{-1}$ near the photosphere (innermost envelope). At some distance from the star the carbonate material condenses and the gas is accelerated again until it reaches a velocity of $\simeq 11 \mathrm{~km} \mathrm{~s}^{-1}$. Ridgway \& Keady (1988) have obtained an inner radius for the condensation of carbonate material of $5 R_{*}$. At $\simeq 11 R_{*}$ refractory $\mathrm{Mg}$ - and/or S-bearing molecular species condense and the gas reaches an expansion velocity of $\simeq 14 \mathrm{~km} \mathrm{~s}^{-1}$. Keady et al. (1988) propose a turbulence in the innermost CSE of $\simeq 5 \mathrm{~km} \mathrm{~s}^{-1}$ and a terminal turbulence velocity of $\simeq 1.0 \mathrm{~km} \mathrm{~s}^{-1}$. Lower values for the terminal turbulence velocity can be found in the literature, e.g., $0.9 \mathrm{~km} \mathrm{~s}^{-1}$ (Huggins \& Healy 1986) and $0.65 \mathrm{~km} \mathrm{~s}^{-1}$ (Skinner et al. 1999).

\subsection{Model Description}

A good approach to the spectral line radiative transfer problem in a CSE is given by the large-scale velocity gradient (LVG) method. It is very fast and produces good results but assumes that the line width of a line is negligible compared to the velocity gradient between very close emitting regions of the CSE. Hence, they cannot be used to accurately model warm regions like the innermost $\operatorname{CSE}\left(T_{K} \simeq 1000-2500 \mathrm{~K}\right)$ where the line width can be larger than the expansion velocity gradient.

The most exact model we can develop should be a nonlocal model that involves both the numerical resolution of the statistical equilibrium and radiative transfer equations. Studies at radio wavelengths have successfully applied this method to CSEs (ALI codes by, e.g., Justtanont et al. 2005; Monte Carlo codes by, e.g., González-Alfonso \& Cernicharo 1997; Crosas \& Menten 1997; Schöier \& Olofsson 2001), although they have needed a significant amount of CPU time even when only a few vibrational and rotational levels have been considered. Nevertheless, the most important reason that led us to reject the use of a non-LTE code is the lack of rovibrational collisional rates quoted in the literature for $\mathrm{C}_{2} \mathrm{H}_{2}$ and $\mathrm{HCN}$. We have identified rovibrational lines of $\mathrm{C}_{2} \mathrm{H}_{2}$ and $\mathrm{HCN}$ involving vibrational levels with energies up to $2200 \mathrm{~cm}^{-1}$ that are created close to the star. In the innermost CSE, the kinetic temperature is high enough to significantly populate these vibrational levels having energies between 3000 and $4000 \mathrm{~cm}^{-1}$ or more. For example, $\mathrm{C}_{2} \mathrm{H}_{2}$ has about 50 vibrational levels below $4000 \mathrm{~cm}^{-1}$. Therefore, a thorough model of the envelope should account for many vibrational and rotational states. The large number of rovibrational levels to be considered makes the problem computationally impractical with these kind of methods. We can significantly reduce the computing time by avoiding exact solutions of the statistical equilibrium equations. We do this by assuming a temperature dependence with radius (supported by previous work or physical considerations) and by allowing several parameters to be free in order to fit the observed lines. A reasonably good estimation to the excitation temperatures could be achieved taking advantage of the large number of observed rovibrational lines, allowing us to estimate also the populations of many rovibrational levels below $2500 \mathrm{~cm}^{-1}$. This handmade processing technique accounts for all the physical phenomena having an influence in the molecular level populations at the nearby environment of each position in the envelope, for example, the collisional rates.

Concerning the geometry of the envelope, modeling the important deviations from spherical symmetry in the innermost CSE requires a large number of parameters that have limited impact in our fits due to the low angular resolution of our observations. Nonetheless, the likely complex velocity field of the gas might introduce some small features in a well-defined region of the emission component of the high-excitation line profiles. Unfortunately, the signal-to-noise ratio is not large enough to unmistakably identify the signs of the inner structure. Moreover, the envelope approaches spherical geometry at large scales. Therefore, we have ignored the complex inner dust structure and assumed spherical symmetry for the whole CSE.

These approximations allow us to solve the problem with onedimensional calculations. We choose a two-dimensional coordinate system with the $y$-axis parallel to the line of sight pointing away from the Earth and the $x$-axis perpendicular to $y$ in an arbitrary direction. Then, we study the evolution of just one ray of light parallel to $y$ for each $x$ and build the final spectrum using the symmetry of the envelope. The emerging intensity at each $x$ is calculated by summing the emission of each region backward from the star, so that the radiation emitted by a region is affected by the optical depth of those in the foreground. Due to its importance in the MIR, dust is included in the calculations and radiatively coupled to the gas. The last step is to multiply the emerging intensity by the point-spread function (PSF) of the telescope, take into account the slit dimensions of the spectrometer, and convolve the resultant flux with the frequency response of the detector. By virtue of simplicity, we have adopted a Gaussian profile for both the PSF and the detector response with the half-power beamwidth 
(HPBW) of the telescope and the width of each channel of the detector as FWHM, respectively.

We have adopted the velocity field structure proposed by Keady et al. (1988), which consists of three different zones: region I starts at the stellar photosphere and ends at the distance of the first dust formation layer, $R_{d 1}$; region II starts at that radius and extends to the position of the second dust formation layer, $R_{d 2}$; finally, the rest of the envelope is region III. We have initially assumed that the two dust formation regions are located at $5 R_{*}$ and $15 R_{*}$ and have a thickness of $1 R_{*}$ (Keady et al. 1988). We allow these values to vary in the model to get the best fit for the dust emission (continuum of the star) and the molecular features. We have divided the envelope into a large number of concentric shells to follow the rapid variation of physical conditions. Using a logarithmic step for the radius increment, most shells are placed in the inner and middle regions (regions I and II), where the temperature and density gradients are more important.

We have tried to find some simple laws for the variation of molecular abundances, density, and gas kinetic and dust grain blackbody temperatures as a function of $r$ that best reproduce the observed continuum and line intensities and profiles. We have assumed that all the temperatures (dust, kinetic, vibrational, and rotational) follow a continuous radial dependence $r^{-\alpha}$, where $\alpha$ could be different for each region of the envelope and for each rovibrational level $\left(\alpha \rightarrow \alpha_{v J}\right)$, depending on the considered temperature. In the case of the dust temperature, we have assumed that $\alpha$ remains constant over the whole dusty CSE. The parameter $\alpha$ (for regions I and II, and for each temperature except the dust temperature) is completely determined through the input of the corresponding temperature at $R_{*}$ and $R_{d 1}$ for region I and $R_{d 1}$ and $R_{d 2}$ for region II. In region I $T_{x}\left(R_{*}\right) / T_{x}\left(R_{d 1}\right)=\left(R_{*} / R_{d 1}\right)^{-\alpha_{x}, \mathrm{I}}$ and in region II $T_{x}\left(R_{d 1}\right) / T_{x}\left(R_{d 2}\right)=\left(R_{d 1} / R_{d 2}\right)^{-\alpha_{x, \mathrm{II}}}$, where $x$ can be dust, kinetic, vibrational, or rotational. However, $\alpha$ in region III cannot be obtained accurately from a modeling of different lines. Consequently, we have assumed $\alpha=1.0$ for all the temperatures in region III (Doty \& Leung 1997). As seen below, this hypothesis is compatible with the fits. On the other hand, the gas density is assumed to satisfy the continuity equation:

$$
\dot{M}=4 \pi r^{2} v_{\exp } \mu m_{\mathrm{H}_{2}} n_{\mathrm{H}_{2}}(r)
$$

where $\mu=\sum m_{i} x_{i} / m_{\mathrm{H}_{2}}, m_{i}$ is the mass of the $i$ th most abundant species, and $x_{i}$ is its abundance with respect to $\mathrm{H}_{2}$. Since the most abundant species after $\mathrm{H}_{2}$ are $\mathrm{He}$ and $\mathrm{CO}$ (with $x \simeq 0.2$ [solar abundance, Cox 2000] and $\simeq 8 \times 10^{-4}$, respectively), then $\mu \simeq 1.4$.

For dust grains, we have assumed a static density profile following an $r^{-2}$ variation law:

$$
n_{d}(r)=\frac{\tau_{\lambda_{0}}}{a_{\lambda_{0}}} \frac{1}{R_{d 1}}\left(\frac{R_{d 1}}{r}\right)^{2},
$$

where $\tau_{\lambda}$ and $a_{\lambda}$ are the optical depth and the absorption of a dust grain at wavelength $\lambda$, respectively, $\lambda_{0}$ is a fixed wavelength, and $\tau_{\lambda_{0}}$ is an input parameter of the model. The wavelength $\lambda_{0}$ that we have adopted is $11 \mu \mathrm{m}$. The dust opacity is derived from the optical properties of AC and $\mathrm{SiC}$ at each wavelength, and the emission is computed from the dust opacity and temperature in each volume element.

The emission and absorption in an elementary integration step are calculated from the adopted velocity field, the corresponding vibrational and rotational temperatures, the $\mathrm{H}_{2}$ density, and the molecular abundances. For the gas, the adopted line frequencies used for the line identification are given in Appendix A, while all the data relevant to line intensities (opacity, dipole moment, and partition function) are in Appendix B.

The line profile is assumed to be Gaussian with a thermal line width over the external CSE because the dust and the gas are expected to expand at their terminal velocity in a steady flow. In contrast, the line width next to the photosphere has not been well determined by previous observations of IRC +10216 . The matter ejection near the star could increase the microturbulent velocity, adding a nonthermal contribution to the line width. In our code, this nonthermal contribution has been implemented as $\Delta v_{m, 1} e^{-(r-1) / \ell}$, where $\Delta v_{m, 1}$ is the nonthermal microturbulent velocity over the photosphere and $\ell$ is a characteristic length ( $r$ and $\ell$ are measured in $R_{*}$ ). Since the lines affected by temperature and/or microturbulence present Gaussian profiles, the resultant line width is $(\Delta v)^{2}=\left(\Delta v_{\text {temperature }}\right)^{2}+\left(\Delta v_{\text {microturbulence }}\right)^{2}$. In order to obtain physically significant parameters from the code, the parameter we have used is the total line width in the photosphere, $\Delta v_{1}$. Highenergy rovibrational lines, which are formed at the photosphere, could carry some information on these parameters.

As many observed lines are modified by many weak features, telluric effects, and overlaps with other lines, the fits derived by the minimization of $\chi^{2}$ would be worse than those obtained here. In addition, the model depends on many parameters, and minimizing the $\chi^{2}$ function for all the selected lines is computationally unrealistic. In fact, we were able to obtain reasonably good eye fits that are in agreement with those derived by minimizing the $\chi^{2}$ function when diminishing the number of parameters (as a first approximation).

However, in the case of the uncertainties of the parameters (see $\S 8)$, we have used a numerical method. Given an observed line, several parameters could similarly affect the synthetic profile, masking their effects and hindering an accurate determination of all their values that reasonably fit the line. Thus, we could calculate the maximum value adopted by a given parameter, by considering it as a function of the others (having imposed a restriction on the synthetic profile with respect to the observed one, for example, setting the function $\chi^{2}$ to be equal to a given number or forcing the synthetic profile to differ from the observed one in less than a given quantity), and by following the gradient of this function. It is also possible to find the minimum value of that parameter by following the field opposite to the gradient. This is a good method to be used for a large number of parameters and for a few lines, since it is possible to approach the maximum (minimum) in several iterations avoiding unnecessary calculations and spending a reasonable amount of CPU time.

In our models we have adopted a stellar temperature of $2330 \mathrm{~K}$, a distance of $180 \mathrm{pc}$, an angular radius for the star of $0.019^{\prime \prime}$ (corresponding to a stellar radius of $735 R_{\odot}$ ), and a mass-loss rate of $2.1 \times 10^{-5} M_{\odot} \mathrm{yr}^{-1}$.

The region between $1 R_{*}$ and $100 R_{*}$ is shielded against Galactic UV photons by the outer dust envelope. Hence, we have considered the radiation field coming only from the star and the dust component of the CSE.

Although the envelope can extend to more than $1000 R_{*}$, the dust in the outermost part of the CSE does not contribute significantly to the continuum flux in the MIR (an envelope with a maximum radius of $600 R_{*}$ divided in 300 shells is enough to find a good fit for the continuum).

The vibrational and rotational temperatures decrease so fast that only the emission and absorption of the low- $J$ levels of the ground vibrational state of $\mathrm{C}_{2} \mathrm{H}_{2}$ and $\mathrm{HCN}$ could be affected by the choice of the external radius in our models (e.g., at $100 R_{*}$ the kinetic temperature is $\simeq 85 \mathrm{~K}$ and the most populated level of $\mathrm{C}_{2} \mathrm{H}_{2}$ is $J \simeq 4-5$; see Fig. 7). The outer radius of the CSE for 


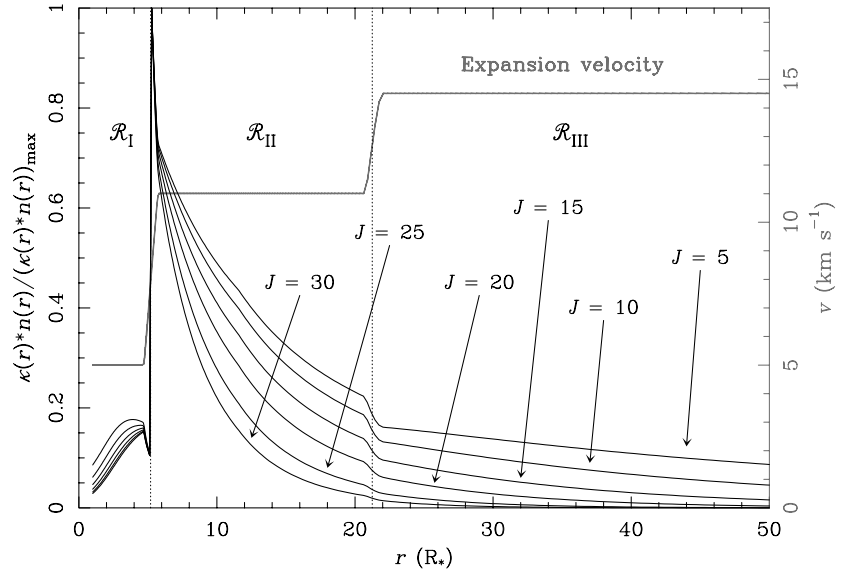

FIG. 7.-Optical depth per unit length integrated over frequency for several lines of the $\nu_{5}\left(\pi_{u}\right) R_{e}$ branch of $\mathrm{C}_{2} \mathrm{H}_{2}$. The optical depth for low- $J$ lines is still large at $50 R_{*}$. However, high- $J$ lines $(J>20)$ will be mainly formed in the inner and middle regions (region I, $\mathcal{R}_{\mathrm{I}}$, and region II, $\mathcal{R}_{\mathrm{II}}$ ). Hence, we can obtain information about the outer envelope (region III, $\mathcal{R}_{\text {III }}$ ) from low- $J$ lines, while high- $J$ lines are sensitive to the physical and chemical conditions near the star. Choosing the correct line can help improve the accuracy of the determination of the parameters in a particular region (see Table 7). The thick gray line represents the velocity field. The sharp increase in opacity at $\simeq 5 R_{*}$ corresponds to the change in $\mathrm{C}_{2} \mathrm{H}_{2}$ abundance in region I found in this work. [See the electronic edition of the Journal for a color version of this figure.]

fitting the lines has been fixed to $300 R_{*}$ since there is no significant modification due to the CSE region between $300 R_{*}$ and $600 R_{*}$. A total of 100 layers were modeled.

The absorbing feature at terminal velocities is mainly produced in region III for low- $J$ rovibrational lines. Optically thin absorbing lines are produced essentially in region II, related to the $(-10$, 10) $\mathrm{km} \mathrm{s}^{-1}$ velocity range in the profiles, setting the absorption maxima at velocities around -8 and $-10 \mathrm{~km} \mathrm{~s}^{-1}$. The innermost region of the CSE (region I), as well as the fact that the dust continuum is largely formed outside of this radius, has little effect in low- $J$ line profiles due to its small angular size compared with the rest of the CSE. The kinetic temperature and density are high enough to assure LTE throughout the envelope for $J \leq 20$ within vibrational levels up to $2 \nu_{5}$ for $\mathrm{C}_{2} \mathrm{H}_{2}$ and up to $2 \nu_{2}$ for $\mathrm{HCN}$. The validity of this assumption has been checked in our models and found to be acceptable. However, high-energy rovibrational levels are populated almost completely in region I, allowing us to derive physical parameters for the regions closest to the star. Consequently, the sensitivity of the observed line profiles to the physical conditions of the gas and the large variation of these parameters across the envelope limit the radial resolution we can achieve for the molecular abundance profiles.

\section{CONTINUUM EMISSION}

The dust properties affect considerably the molecular excitation due to radiative coupling between the dust and the gas. P Cygni profiles arise in all the lines created in the inner CSE. In particular, the $\mathrm{C}_{2} \mathrm{H}_{2}$ and $\mathrm{HCN}$ lines show this kind of line shape (see Figs. 1-6). The line profile depends on the dust parameters such as the dust temperature, composition, size of the dust grains, and absorption and scattering cross sections.

For the wavelength range under consideration (MIR, $\lambda \simeq 11-$ $14 \mu \mathrm{m})$, the size of the grains has a small effect on the continuum. This parameter can be neglected at larger wavelengths (FIR and radio) but is much more important in the near-infrared (NIR) range (Ivezić \& Elitzur 1996), i.e., $\lambda \leq 1-2 \mu \mathrm{m}$. We have assumed that dust grains are spheres with a constant radius not larger than $0.1 \mu \mathrm{m}$ and calculated their opacity using Mie theory (e.g., Hoyle \& Wickramasinghe 1991). According to the theory, the scattering cross section is small compared to the absorption cross section for dust grains with diameter small compared to the wavelength of radiation. Since we are considering $\lambda \simeq 11-14 \mu \mathrm{m}$ radiation, we ignore scattering. The composition of the dust grains is guessed to be $\mathrm{AC}$ and $\mathrm{SiC}$ (see $\S 3.1$ ). The complex refractive index of AC as a function of $\lambda$ has been taken from Rouleau $\&$ Martin (1991). Laboratory works on SiC (Mutschke et al. 1999) suggest that $\mathrm{SiC}$ is probably crystalline in space. Unfortunately, the difficulties in measuring the optical constants for crystalline $\mathrm{SiC}$ have led us to adopt the laboratory data for the refractive index of amorphous $\mathrm{SiC}$ in our models. For the dust temperature, $T_{d}$, we initially assume the $r^{-0.4}$ dependence, proposed by Ridgway $\&$ Keady (1988). Actually, the $T_{d}$ dependence on $r$ is steeper in the innermost CSE than at greater distances because the opacity of the dust grains is larger at higher frequencies, i.e., closer to the star. We have adopted the power law shown above as an approximation. The exponent is a very sensitive parameter and can be determined with a high accuracy (see $\S 8$ ). Finally, concerning the dust optical depth, $\tau_{\lambda}$, the latter authors derived a value $\tau_{\lambda} \simeq 1$ at $11 \mu \mathrm{m}$, Ivezić \& Elitzur (1996) obtained $\tau_{\lambda} \simeq 0.32-0.40$ at $10 \mu \mathrm{m}$, and Monnier et al. (2000) obtained $\tau_{\lambda} \simeq 0.66$ at $11.15 \mu \mathrm{m}$.

In this work we have fitted the continuum data of IRC +10216 obtained by ISO SWS, adjusting the dust parameters as described above. The ISO observations used for that purpose are those quoted by Cernicharo et al. (1996, 1999). We have assumed that the difference between the stellar phases of the observations (see $\S 2$ ) with ISO SWS and TEXES at the Infrared Telescope Facility (IRTF) does not introduce any important effect on dust and molecular properties and that the derived $T_{d}$ from the ISO data is similar to those prevailing at the moment of the TEXES observations.

We have fitted the continuum between 7 and $27 \mu \mathrm{m}$. Fitting to shorter wavelength $I S O$ data is inappropriate because scattering will be important and the model does not include scattering. The brightness of the central star has a small impact on the observed continuum. The maximum contribution to the continuum from the star is at $5.31 \mu \mathrm{m}\left(F_{\nu} \simeq 1075 \mathrm{Jy}\right)$, and the emission at $10 \mu \mathrm{m}$ is $\simeq 1.73$ times lower. A small modification of the stellar temperature does not significantly affect the flux emerging from the source. Note, however, that a large change in the stellar temperature could modify the physical and chemical properties of the envelope and, hence, of the emergent flux. In any case, it seems obvious that the observed MIR and FIR emission in IRC +10216 comes mainly from the dusty envelope.

We obtain from our analysis that the inner and outer dust formation shells are located at radii $5.2_{-0.5}^{+0.6} R_{*}$ and $21 R_{*} \pm 3 R_{*}\left(0.1^{\prime \prime}\right.$ and $0.4^{\prime \prime}$ ), respectively (see Fig. 7, Table 7, and $\S 8$ ). A value $R_{d 1}=5.2 R_{*}$ is derived from fitting only the continuum. However, $R_{d 2}$ can be determined with more or less precision through fitting several molecular lines, while the continuum of the star displays little information about this outer dust formation shell. Studying how changes to the dust density, $n_{d}$, affect $R_{d 2}$ led us to very inaccurate results because the variation of $n_{d}$ over this shell only slightly affects the continuum.

Several studies have determined $R_{d 1}$ since the discovery of IRC +10216 . Keady et al. (1988) proposed that $R_{d 1} \simeq 3 R_{*}$ equivalent to $\simeq 0.057^{\prime \prime}$, with a stellar radius of $\simeq 970 R_{\odot}$ and a distance to the star of $200 \mathrm{pc}$. Later, Ridgway \& Keady (1988) found $R_{d 1} \simeq$ $5 R_{*}$ and an angular stellar radius of $\simeq 0.019^{\prime \prime}$, implying an angular radius of the inner dust formation zone, $\alpha_{d 1}$, of $\simeq 0.095^{\prime \prime}$. Monnier et al. (2000) found $\alpha_{d 1} \simeq 0.15^{\prime \prime}$ at a distance of $135 \mathrm{pc}$ and $\alpha_{*} \simeq$ $0.022^{\prime \prime}$, meaning that $R_{d 1} \simeq 6.8 R_{\odot}$, and Ivezić \& Elitzur (1996) determined $\alpha_{d 1}$ to be $\simeq 0.22^{\prime \prime}$ at maximum luminosity and $\simeq 0.15^{\prime \prime}$ at minimum luminosity. Consequently, our result is in good agreement with the values quoted in the literature. For $R_{d 2}$, the angular 
TABLE 2

Identified and Modeled Lines of $\mathrm{C}_{2} \mathrm{H}_{2}, \mathrm{H}^{13} \mathrm{CCH}, \mathrm{HCN}$, And $\mathrm{H}^{13} \mathrm{CN}$

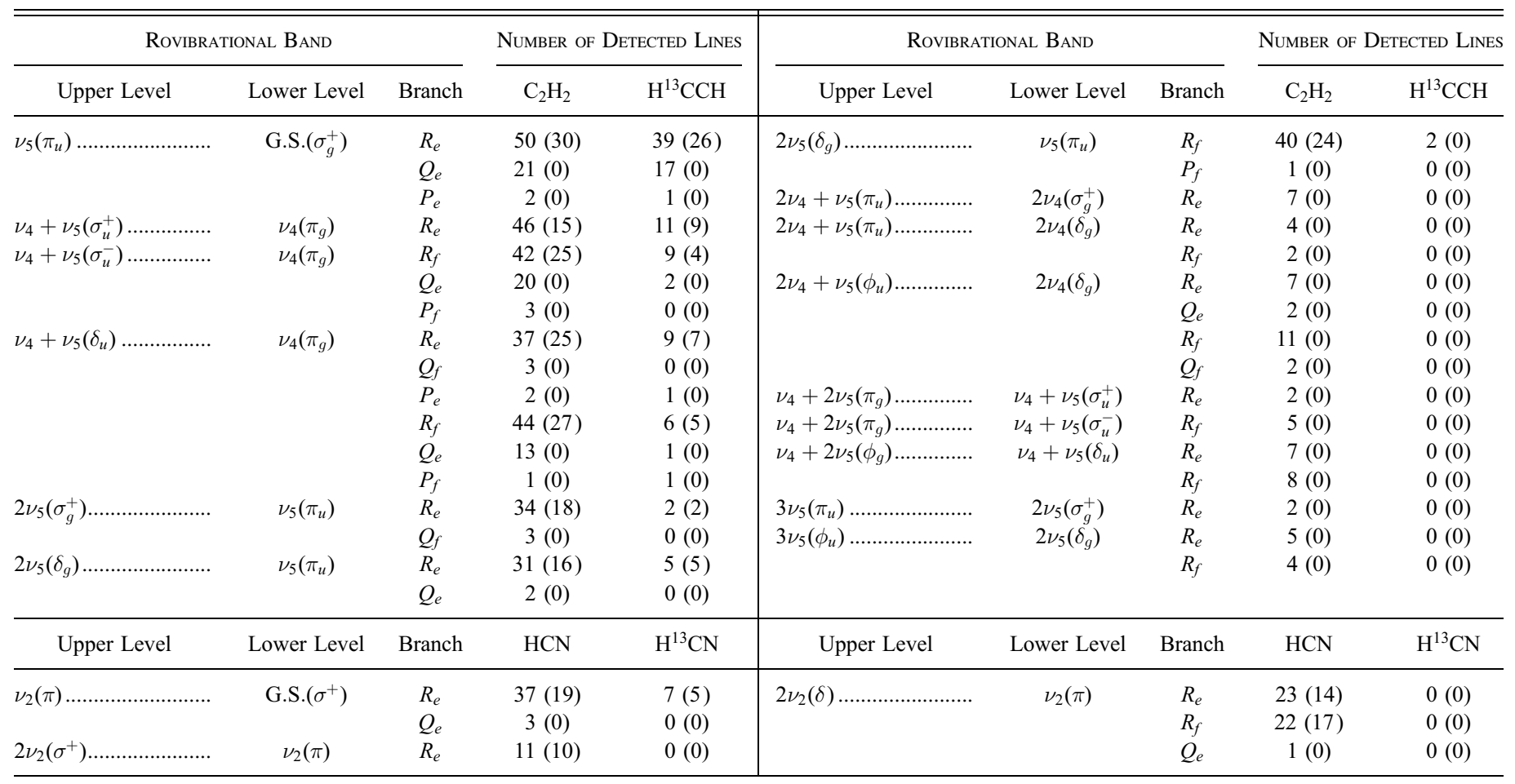

Notes.- The numbers without parentheses are the detected lines, while the numbers within parentheses are the modeled lines. Many lines for each band are

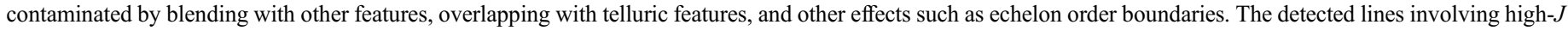

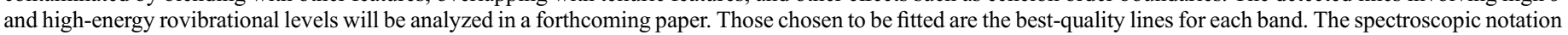
used is described in Appendix A.

radius of the outer dust formation zone, Keady et al. (1988) found $R_{d 2} \simeq 14 R_{*}\left(\alpha_{d 2} \simeq 0.32^{\prime \prime}\right)$. We derived $\alpha_{d 2} \simeq 0.40^{\prime \prime}$, a larger value than found by Keady et al. (1988) and Keady \& Ridgway (1993).

The optical depth derived with our model at $11 \mu \mathrm{m}$ is 0.7 , similar to that proposed by Monnier et al. (2000), $\tau(\lambda=11.15 \mu \mathrm{m})=$ 0.66 , and larger than the value proposed by Ivezić \& Elitzur (1996), who derived $\tau(\lambda=10 \mu \mathrm{m}) \simeq 0.32-0.40$. On the other hand, the value for the temperature of the innermost dust formation shell, $T_{d 1}$, derived in this work is $850 \pm 25 \mathrm{~K}$ (see $\S 8$ and Table 7 for a discussion on the errors), lower than that obtained by Ridgway $\&$ Keady $\left(1988 ; T_{d 1}=1040 \pm 100 \mathrm{~K}\right)$ and Groenewegen (1997; $\left.T_{d 1}=1075 \pm 50 \mathrm{~K}\right)$, similar to that obtained by Monnier et al. $\left(2000 ; T_{d 1}=860 \mathrm{~K}\right)$, and higher than that obtained by Ivezić \& Elitzur $\left(1996 ; T_{d 1}=750 \pm 50 \mathrm{~K}\right)$. The exponent of the $T_{d}$ law is derived to be 0.39 , quite similar to that proposed by Keady et al. (1988).

The best fit to the dust composition is 95\% AC and 5\% amorphous $\mathrm{SiC}$. Modifying the proportion of $\mathrm{SiC}$ changes the ratio of the predicted flux at $11 \mu \mathrm{m}$, with respect to the rest of the continuum. Nevertheless, this change in composition could be balanced by modifying the optical depth and dust temperature at $R_{d 1}$. Fitting the whole continuum is necessary to get more realistic percentages.

Taking into account the different observing periods and the wavelength coverage in these different data sets, we consider that our estimates of $T_{d}$ and opacity in $R_{d 1}$ are representative of the physical conditions in IRC +10216 .

\section{5. $\mathrm{C}_{2} \mathrm{H}_{2}$ MODELING}

In order to obtain the abundance of $\mathrm{C}_{2} \mathrm{H}_{2}$ and the physical parameters of the CSE, we have selected several sets of $\mathrm{C}_{2} \mathrm{H}_{2}$ lines that could be sensitive to the derived parameters (see Table 2 and
Fig. 7). The assumed gas velocity profiles and computed line profiles are in very good agreement (see Fig. 8). The $\mathrm{C}_{2} \mathrm{H}_{2}$ abundance and physical conditions from the fits are listed in the following subsections.

\subsection{Velocity Profile}

The simultaneous modeling of different $J$ rovibrational transitions of the same band requires a three-region velocity field (Keady et al. 1988). As can be seen in Figure 7, lines with higher energy are formed closer to the photosphere. Consequently, the effect of the expansion on the line shape changes with $J$. In many cases, a variation of the velocity in one of these regions modifies the line shapes significantly. For example, the gas velocities in regions II and III control the position of the intensity minimum, so that lines formed closer to the star represent the bulk absorption at lower velocities than the lines formed at larger distances.

On the other hand, the model is very insensitive to variations in the thickness of the acceleration shells ( $\operatorname{see} \S 8)$. The line widths in these regions are similar to the velocity increasing in the acceleration zones and hence hide the acceleration effects on the profiles. The small spatial extent of the innermost region and the limited angular resolution of our data prohibit a better determination of the physical and chemical conditions in region I. We have found that the best fit to the lines can be achieved with the following velocity profile (see Fig. 7):

$$
v_{e}(r)= \begin{cases}5, & 1 \leq r / R_{*}<4.7, \\ 5-11^{*}, & 4.7 \leq r / R_{*}<5.7, \\ 11, & 5.7 \leq r / R_{*}<20.75, \\ 11-14.5^{*}, & 20.75 \leq r / R_{*}<21.75, \\ 14.5, & 21.75 \leq r / R_{*},\end{cases}
$$




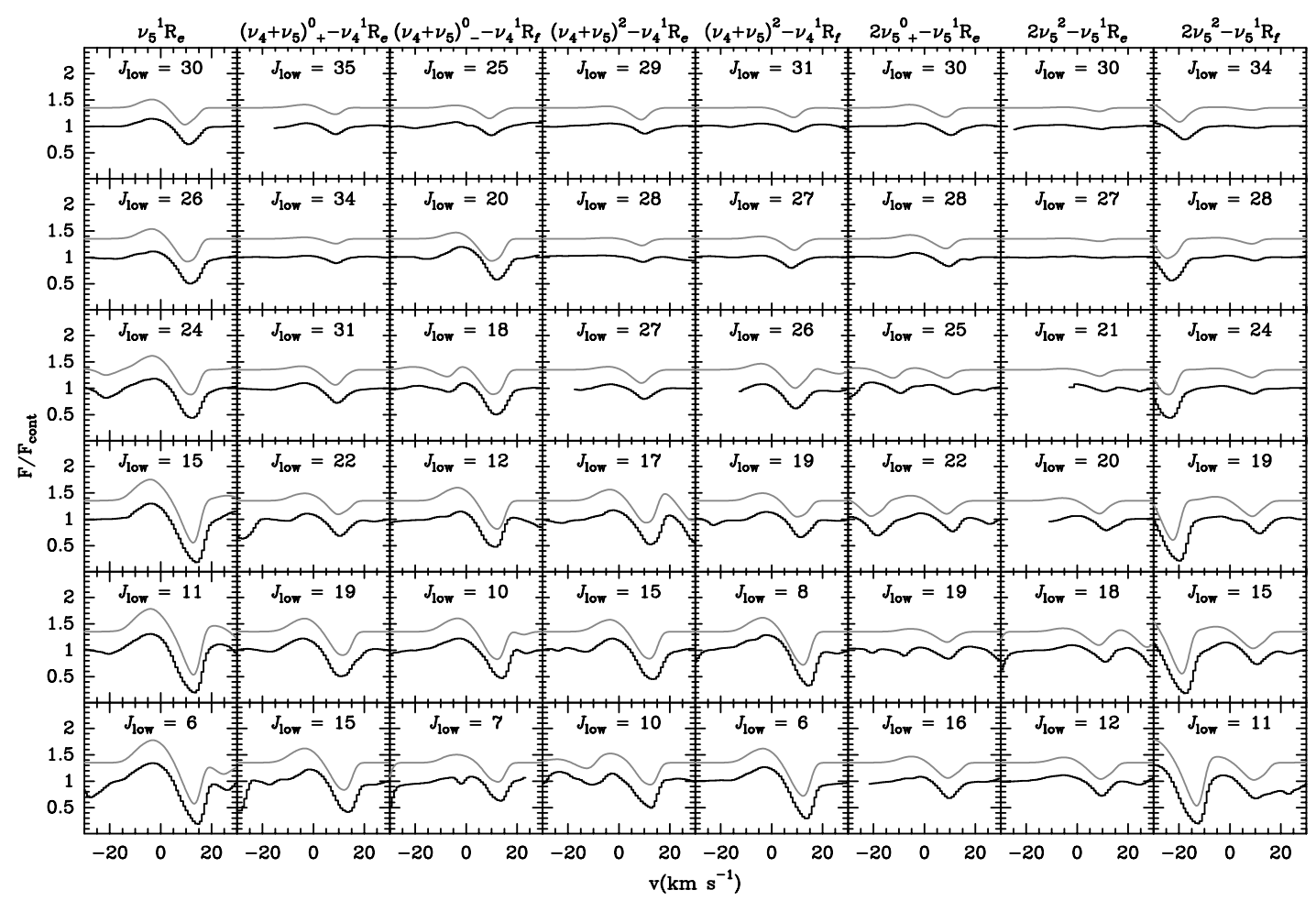

FIG. 8.-Fits of some lines of $\mathrm{C}_{2} \mathrm{H}_{2}$. The black lines are the observed spectra, while the gray lines are the fits. The data have been corrected from observational frequency deviations adding/suppressing the mean difference between the observational and the laboratory frequency of many lines (see $\S 2$ ). The existing discrepancies are small and can be assumed as blends with other lines and/or observational errors (the spectroscopic notation is described in Appendix A). [See the electronic edition of the Journal for a color version of this figure.]

where the asterisk means constant velocity gradient $d v_{e} / d r$ between the inner and outer radii of the corresponding zone.

\subsection{Line Width}

When microturbulence is included in the calculations, the emission from region I spreads and the intensity of the lines reaches its maximum at higher velocities (toward lower frequencies), compared with the fits without microturbulence. Unfortunately, fits for low- and intermediate- $J$ rovibrational levels are relatively insensitive to the total line width in the photosphere, $\Delta v_{1}$, and the characteristic length, $\ell$. A substantial change in the fitted profiles requires unrealistic values for these parameters. Reasonable values, for example, are $\Delta v_{1}=5 \mathrm{~km} \mathrm{~s}^{-1}$ and $\ell=1.5 R_{*}$. The value of $\ell$ is acceptable due to the physical phenomena thought to occur near the photosphere, i.e., a moderate pulsation of the central star and the appearance of sound waves (Bowen 1988; Pijpers \& Hearn 1989; Pijpers \& Habing 1989). However, the fits obtained with these parameters are not more accurate than those calculated without microturbulence. In fact, when choosing $\Delta v_{1}=30 \mathrm{~km} \mathrm{~s}^{-1}$ and $\ell=1.5 R_{*}$, the emission can be fitted more accurately. We discuss this further in $\S 8$.

\subsection{Kinetic Temperature, $T_{K}$}

$\mathrm{C}_{2} \mathrm{H}_{2}$ low- and intermediate-energy levels are highly populated in each vibrational level, and many of the rovibrational transitions in which they are involved are optically thick and therefore hide information (e.g., low- $J$ transitions in the fundamentals in regions I and II). However, there are still many lines in LTE that are not optically thick in the hot bands over the whole envelope and in the fundamental band in regions II and III (see Fig. 7). We can determine the kinetic temperature profile by fitting simultaneously several low- and intermediate- $J$ lines from the fundamental band and relatively low energy hot bands. The relationship between the intensities of adjacent rovibrational lines in LTE within a given band allows us to derive $T_{K}$ immediately. In doing so, it is necessary to derive the vibrational temperatures and the molecular abundances at the same time as $T_{K}$. We discuss this determination in $\S 5.5$.

We have fixed the kinetic temperature at $r=1 R_{*}$ equal to the temperature of the central star. For regions I and II, $T_{K}$ at $R_{d 1}$ and $R_{d 2}$ are free parameters that completely determine the value of the exponent. The temperature exponent obtained is 0.58 for both regions I and II, while the fits support the assumption of $\alpha=1.0$ over region III.

The kinetic temperature profile derived from the best fit to all $\mathrm{C}_{2} \mathrm{H}_{2}$ lines is

$$
T_{K}(r)= \begin{cases}2330\left(r / R_{*}\right)^{-0.58}, & \text { region I, } \\ 900\left(r / R_{d 1}\right)^{-0.58}, & \text { region II, } \\ 400\left(r / R_{d 2}\right)^{-1.00}, & \text { region III. }\end{cases}
$$

\subsection{Rotational Temperatures, $T_{\text {rot }}$}

An accurate estimate of the rotational temperatures requires a sophisticated model including molecular parameters, such as collisional rates between rovibrational levels, that are currently unavailable. Therefore, we have used our data to derive a first estimate of rotational temperatures for all rovibrational transitions in the three zones defined above. The procedure to derive $T_{\text {rot }}$ profiles is as follows:

1. $T_{K}$ and the vibrational temperatures are derived by modeling the rovibrational transitions that involve both low- and intermediateenergy rotational levels (see $\S 5.3$ ).

2. Initially, all the high-energy rovibrational levels are considered to be in LTE. 
TABLE 3

Ad Hoc Rotational Temperatures of $\mathrm{C}_{2} \mathrm{H}_{2}$ and $\mathrm{HCN}$

\begin{tabular}{cccccc}
\hline \hline & $\mathrm{C}_{2} \mathrm{H}_{2}$ & & & \multicolumn{2}{c}{$\mathrm{HCN}$} \\
\cline { 1 - 3 } \cline { 5 - 6 } G.S. $\left(\sigma_{g}^{+}\right)$ & $\nu_{4}\left(\pi_{g}\right)$ & $\nu_{5}\left(\pi_{u}\right)$ & & G.S. $\left(\sigma^{+}\right)$ & $\nu_{2}(\pi)$ \\
\hline $35(900,50)$ & $26(620,200)$ & $20(900,200)$ & & $20(900,250)$ & $25(250,35)$ \\
$36(900,60)$ & $32(425,100)$ & $26(900,150)$ & & $24(640,85)$ & \\
& $34(425,100)$ & $32(620,200)$ & &
\end{tabular}

Notes.-Ad hoc rotational temperatures of $\mathrm{C}_{2} \mathrm{H}_{2}$ and $\mathrm{HCN}$ for the lowest vibrational levels. The numbers are shown according to the nomenclature $J\left[T_{\text {rot }}\left(R_{d 1}\right), T_{\text {rot }}\left(R_{d 2}\right)\right]$, where $J$ is the rotational level requiring an ad hoc $T_{\text {rot }}$. The temperatures are expressed in $\mathrm{K}$ (see the text for an explanation for the radial dependence). The rest of the rotational temperatures have a smooth dependence on $J$. We have only considered those rotational levels involved in rovibrational levels up to $J=35-40$ (depending on the band). Maybe new ad hoc $T_{\text {rot }}$ should be included for higher $J$ levels.

3. We vary $T_{\text {rot }}$ for all the high- $J$ levels in each vibrational state to fit the observed line profiles. $T_{\text {rot }}$ is considered to depend on $J$ as a second-degree polynomial.

4. In the next iteration, step 3 is repeated for the next rovibrational transition of the band under consideration.

At each step, all line intensities are recalculated because a change in the rotational temperature of one of them implies a variation in line opacities. As the transitions involve higher energy levels, the variation in the opacities of the fitted transitions becomes lower and lower. The process is repeated until convergence is reached for each band. The remaining lines to fit belong to rovibrational transitions involving vibrational levels with higher energies. Running the code two or three times is usually enough to fit the line correctly with a CPU time of less than $10 \mathrm{~s}$ per line. Unfortunately, fitting lines of hot bands sometimes requires repeating the fitting process for lines involving lower energy vibrational levels since the upper level of an optically thick transition can coincide with the lower level of an optically thin one.

As discussed above, $T_{\text {rot }}$ may be considered equal to the kinetic temperature in the innermost zones for low- and intermediate- $J$ levels in lower energy vibrational states. However, for high- $J$ levels in high-energy vibrational states, we have found that $T_{\text {rot }}$ is below $T_{K}$, even near the photosphere. In addition, for some specific transitions, it has been necessary to include ad hoc rotational temperatures in order to match the data (see Table 3). It is common for two or three important ad hoc rotational temperatures to be found in a single vibrational level below $J \simeq 30-35$. These anomalous rotational temperatures are unrelated to frequencies having larger telluric interference, and there are no apparent instrumental effects. Moreover, high- $J$ lines are in an optically thinner part of the atmosphere than low- $J$ ones, and the latter seem to be under LTE with high accuracy (see Fig. 9). Therefore, these effects could reflect real physical processes such as collisional rates, radiative selective pumping effects, or overlaps of these lines with other spectral features (see Fonfría Expósito et al. 2006).

We have found that $T_{\text {rot }}$ for levels below $J \simeq 20-30$ follows $T_{K}$ (depending on the vibrational level). However, for high- $J$ lines, $T_{\text {rot }}$ is systematically lower than $T_{K}$. This fact seems to indicate that the physical conditions over the inner CSE are not appropriate to maintain high- $J$ levels under LTE, as we assumed in $\S 3.2$.

\subsection{Vibrational Temperatures}

The vibrational temperatures were determined from a fitting of line intensities, rather than by non-LTE radiative transfer cal-

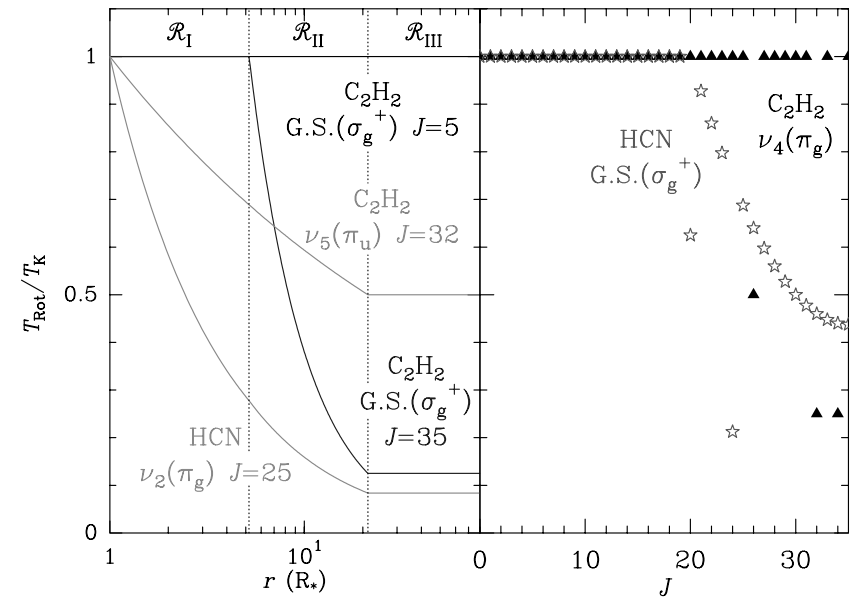

FIG. 9.- Rotational temperatures for some $\mathrm{C}_{2} \mathrm{H}_{2}$ and $\mathrm{HCN}$ levels depending on the radius (left) and rotational temperatures for some $\mathrm{C}_{2} \mathrm{H}_{2}$ and $\mathrm{HCN}$ vibrational levels at $r=R_{d 2}$ depending on $J$ (right). The rotational temperature is less than or equal to the kinetic temperature throughout the CSE (left). Fitting the rovibrational transitions sometimes needs the introduction of ad hoc rotational temperatures, whose dependence usually decreases smoothly with $J$ (right). [See the electronic edition of the Journal for a color version of this figure.]

culations, but we can explain the derived populations with radiative pumping.

Some excited levels of $\mathrm{C}_{2} \mathrm{H}_{2}$ are connected to the ground state only through collisions and radiative cascades ( $\nu_{4}$, Raman active), while others are connected by both collisions and direct absorption of photons ( $\nu_{5}, \mathrm{IR}$ active). Near the star, collisions with $\mathrm{H}_{2}$ and He could play a role in the pumping of the low-energy vibrational levels. Moreover, selection rules for $\mathrm{C}_{2} \mathrm{H}_{2}$ only allow radiative transitions from gerade to ungerade vibrational states and vice versa (Fig. 10).

Consequently, the pumping of low-energy vibrational levels could occur through absorption of NIR photons emitted by both the star and the dust. These photons will pump $\mathrm{C}_{2} \mathrm{H}_{2}$ from the ground state to the strongest stretching mode, $\nu_{3}\left(\sigma_{u}^{+}\right)$, and its combination bands, $\nu_{3}+\nu_{4}\left(\pi_{u}\right), \nu_{3}+\nu_{5}\left(\pi_{g}\right)$, and $\nu_{2}+\nu_{4}+\nu_{5}\left(\sigma_{u}^{+}\right)$ (the notation is discussed in Appendix A), followed by radiative decay to the bending modes (Cernicharo et al. 1999). These infrared transitions are strong $\left[\nu_{3}\left(\sigma_{u}^{+}\right)\right]$, medium $\left[\nu_{3}+\nu_{4}\left(\pi_{u}\right)\right.$, $\left.\nu_{3}+\nu_{5}\left(\pi_{g}\right)\right]$, and very strong $\left[\nu_{2}+\nu_{4}+\nu_{5}\left(\sigma_{u}^{+}\right)\right]$, as indicated by Mandin et al. (2005) and Herzberg (1989b, p. 290).

Radiative pumping through $\nu_{3}\left(\sigma_{u}^{+}\right)$and its combination bands is only effective in the innermost region. Farther out, absorption of MIR photons coming from the star and the dust pumps $\mathrm{C}_{2} \mathrm{H}_{2}$ from the ground state to $\nu_{5}$ and from $\nu_{4}$ and $\nu_{5}$ to their combination band, $\nu_{4}+\nu_{5}$, and other vibrational levels, as overtones. For $r>20 R_{*}$, the available NIR photons are strongly reduced due to dust absorption. However, pumping through $\nu_{5}\left(\pi_{u}\right)$ is efficient over the whole CSE. Hence, we could expect vibrational temperatures, $T_{\mathrm{vib}}$, to be out of LTE as the distance from the star increases.

To estimate $T_{\text {vib }}$, we have selected some low- $J$ lines from each observed vibrational band. As the vibrational excitation temperature, $T_{\mathrm{ev}}$, depends on $T_{\mathrm{vib}}$ and controls the population of the upper vibrational level with respect to the lower one, the vibrational temperatures are coupled to the abundance of the molecular species under consideration. Hence, an iterative procedure involving $T_{K}$, $T_{\mathrm{ev}}$ (involving $T_{\mathrm{vib}}$ ), and the abundance is required to derive (also fitting the observed rovibrational transitions) the populations of all the low- and intermediate- $J$ rotational levels within vibrational levels with low and moderate energy, which are under LTE. So, as 


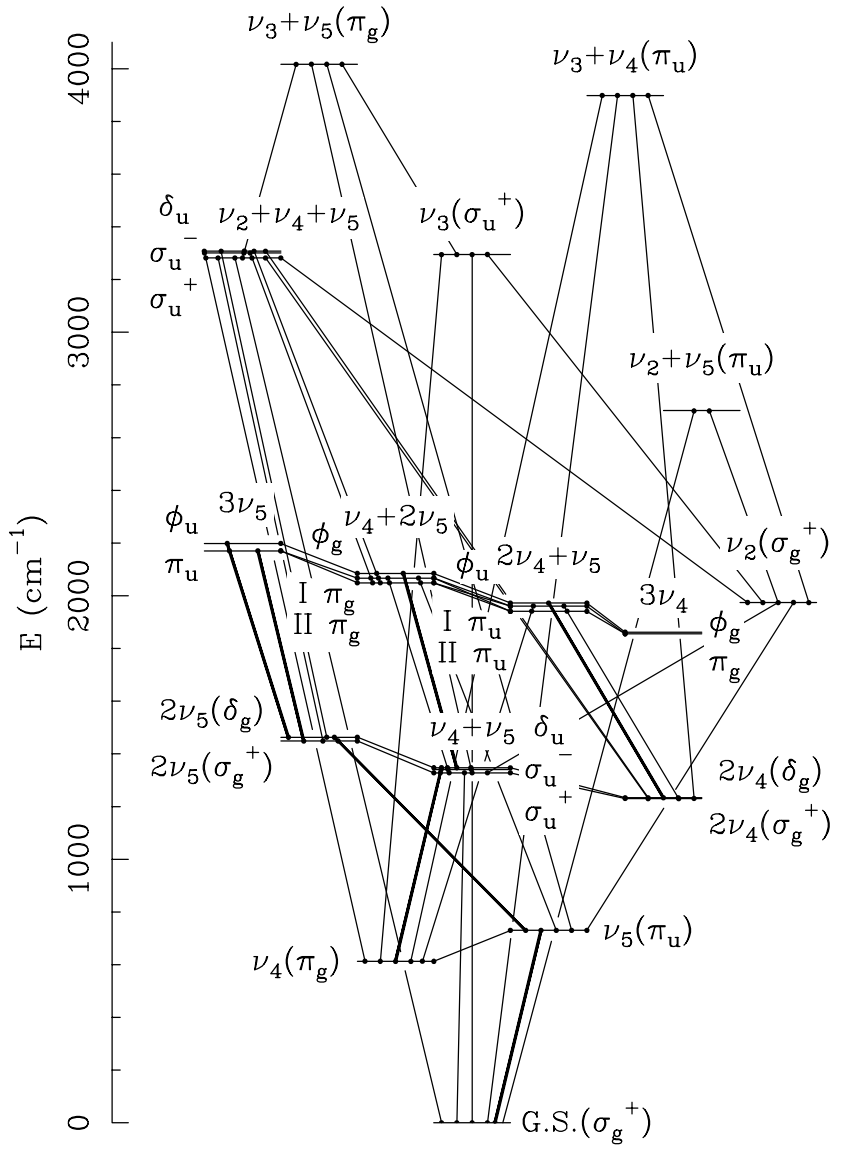

FIG. 10.-Energy of the vibrational levels of $\mathrm{C}_{2} \mathrm{H}_{2}$. The allowed radiative transitions are shown (only the perpendicular transitions with $\Delta v_{4}= \pm 1$ and $\Delta v_{5}=$ \pm 1 , and the most intense parallel ones). This scheme also represents the ${ }^{13} \mathrm{C}_{2} \mathrm{H}_{2}$ vibrational level structure. The vibrational levels for $\mathrm{H}^{13} \mathrm{CCH}$ are similar, but they do not express $g$ or $u$. Some infrared inactive modes of $\mathrm{C}_{2} \mathrm{H}_{2}$ are weakly infrared active for $\mathrm{H}^{13} \mathrm{CCH}$ (i.e., the $\nu_{4}$ mode described by Di Lonardo et al. 2002). The thin lines are allowed transitions, while the thick lines are observed transitions.

we show in $\S 5.6$, the first step in this procedure is to estimate the abundances over the whole envelope. The resulting $T_{\text {vib }}$ for $\mathrm{C}_{2} \mathrm{H}_{2}$ are shown in Table 4, having been defined $T_{\text {vib }}$ with reference to the lower vibrational state (e.g., $T_{\text {vib }}($ G.S. $) \equiv T_{\text {vib }}\left[\nu_{4}\left(\pi_{g}\right)-\right.$ G.S. $\left.\left(\sigma_{g}^{+}\right)\right]$ for $\left.\mathrm{C}_{2} \mathrm{H}_{2}\right)$. Figures 11 and 12 show the derived population, $P_{i}$, and $T_{\mathrm{ev}}$ of the observed vibrational levels, respectively (see the caption of Fig. 11 for the definitions of $P_{i}$ ).

The analysis of the derived vibrational temperatures and populations (compared with those under LTE) indicates the following:

1. In the innermost envelope (region I), the population of the ground state is smaller than for levels $\nu_{4}$ and $\nu_{5}$ because they are doubly degenerate ( $e$ and $f$ parities) while the ground state is an $e$ vibrational level. $T_{K}$ and the strong radiation field near the star are able to pump many molecules to high-energy levels [see Fig. 11; $2 \nu_{5}\left(\delta_{g}\right)$, for example, is at $\left.\simeq 2100 \mathrm{~K}\right]$.

2. Level $\nu_{4}\left(\pi_{g}\right)$ is a metastable vibrational level (see Fig. 10). It is not radiatively connected to the ground state through electric dipole transitions. Collisions and/or radiative cascades are its main pumping mechanisms.

3. Almost all vibrational levels, except $\nu_{5}\left(\pi_{u}\right)$ and $2 \nu_{5}\left(\sigma_{g}^{+}\right)$, can be considered to be in LTE in region I. Level $\nu_{5}\left(\pi_{u}\right)$ is out of LTE because of the strength of the radiative connection to the ground state (the dipole moment of the transition is $\mu \simeq 0.31 \mathrm{D}$; Jacquemart et al. 2001). The population of $2 \nu_{5}\left(\sigma_{g}^{+}\right)$departs from LTE over the whole CSE, even near the stellar surface
TABLE 4

Vibrational Temperatures of $\mathrm{C}_{2} \mathrm{H}_{2}$

\begin{tabular}{|c|c|c|c|c|c|c|c|}
\hline Vibrational Level & $P_{i}$ & $\begin{array}{c}T_{1} \\
(\mathrm{~K})\end{array}$ & $\alpha_{1}$ & $\begin{array}{c}T_{2} \\
(\mathrm{~K})\end{array}$ & $\alpha_{2}$ & $\begin{array}{c}T_{3} \\
(\mathrm{~K})\end{array}$ & $\alpha_{3}$ \\
\hline S. & $P_{0}$ & 2330 & 0.58 & 900 & 0.19 & 685 & 1.00 \\
\hline$\nu_{4}\left(\pi_{q}\right)$. & $P_{1}$ & 2330 & 1.66 & 150 & 0.43 & 82 & 1.00 \\
\hline$\nu_{5}\left(\pi_{u}\right)$ & $P_{2}$ & 2330 & 0.58 & 900 & 0.26 & 625 & 1.00 \\
\hline $2 \nu_{4}\left(\sigma_{q}^{+}\right) \ldots \ldots \ldots \ldots \ldots \ldots \ldots$ & $P_{3}$ & 2330 & 0.58 & 900 & 0.58 & 400 & 1.00 \\
\hline $2 \nu_{4}\left(\delta_{g}\right) \ldots \ldots \ldots \ldots \ldots \ldots \ldots$ & $P_{4}$ & 2330 & 0.58 & 900 & 0.58 & 400 & 1.00 \\
\hline$\nu_{4}+\nu_{5}\left(\sigma_{u}^{+}\right) \ldots \ldots \ldots \ldots \ldots$ & $P_{5}$ & 2330 & 0.58 & 900 & 0.58 & 400 & 1.00 \\
\hline$\nu_{4}+1$ & $P_{6}$ & 2330 & 0.58 & 900 & 0.58 & 400 & 1.00 \\
\hline$\nu_{4}+\nu_{5}\left(\delta_{u}\right) \ldots \ldots \ldots \ldots$ & $P_{7}$ & 500 & 0.98 & 100 & 0.16 & 80 & 1.00 \\
\hline $2 \nu_{5}\left(\sigma_{q}^{+}\right) \ldots \ldots \ldots \ldots \ldots \ldots \ldots$ & $P_{8}$ & 2330 & 0.58 & 900 & 1.40 & 125 & 1.00 \\
\hline$T_{K}$ & $\ldots$ & 2330 & 0.58 & 900 & 0.58 & 400 & 1.00 \\
\hline
\end{tabular}

Notes.-Vibrational temperatures of $\mathrm{C}_{2} \mathrm{H}_{2} . T_{1}$ is the vibrational temperature very near the stellar surface, $T_{2}$ is at the inner dust formation shell $\left(r=5.2 R_{*}\right)$, and $T_{3}$ is at the outer dust formation shell $\left(r=21.2 R_{*}\right)$. Parameters $\alpha_{1}, \alpha_{2}$, and $\alpha_{3}$ are the exponents of the temperature power law $\left(r^{-\alpha}\right)$ in regions I, II, and III, respectively. Note that the vibrational temperature of level $\nu_{4}+\nu_{5}\left(\delta_{u}\right)$ is very low even close to the stellar surface. $T_{K}$ has been included in the last row of the table to allow quick comparisons. The vibrational temperatures refer to the lower vibrational level.

$\left(P_{8} / P_{7}<P_{8} /\left.P_{7}\right|_{\text {LTE }}\right)$, because it is connected radiatively with $\nu_{5}\left(\pi_{u}\right)$ [the dipole moment of the transition $2 \nu_{5}\left(\sigma_{g}^{+}\right) \leftrightarrow \nu_{5}\left(\pi_{u}\right)$ is similar to that of $\nu_{5}\left(\pi_{u}\right) \leftrightarrow$ G.S. $\left.\left(\sigma_{g}^{+}\right)\right]$.

4. The radial dependence of the population of all the vibrational levels changes in region II with respect to region I. The

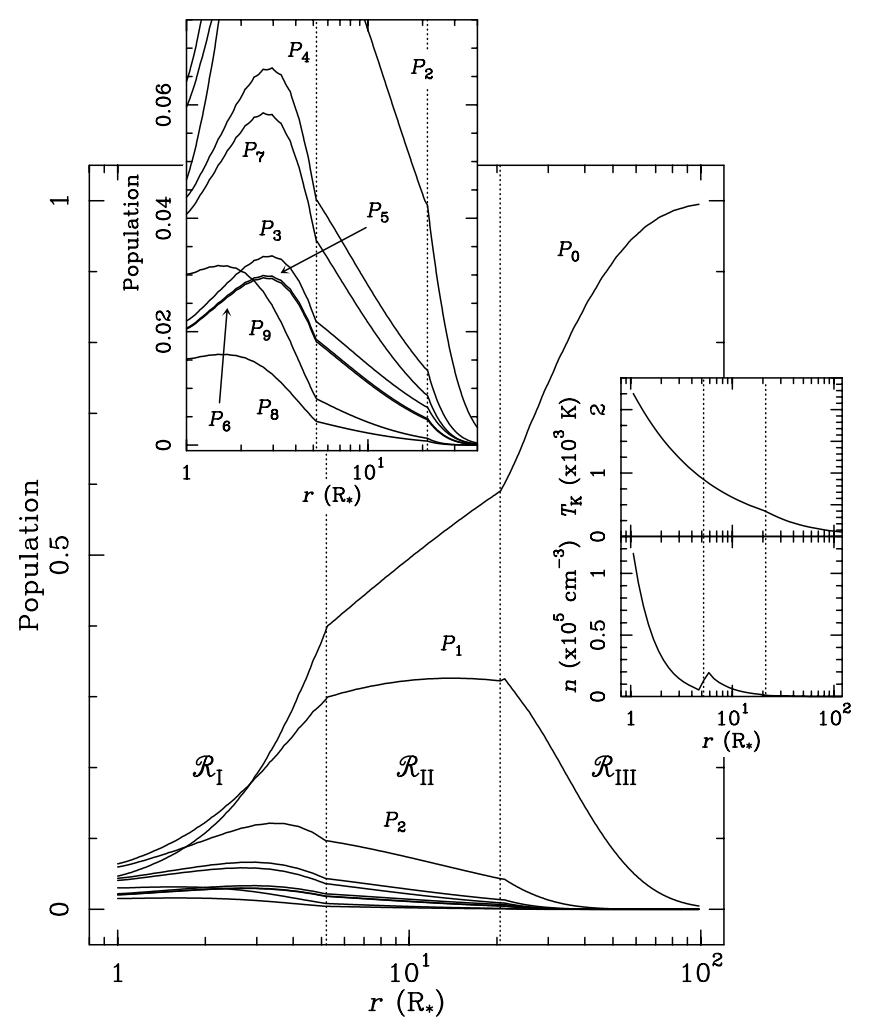

Fig. 11.-Population, $P$, of the vibrational levels of $\mathrm{C}_{2} \mathrm{H}_{2}$. They are labeled as follows: $P_{0} \equiv P\left[\right.$ G.S. $\left.\left(\sigma_{g}^{+}\right)\right], P_{1} \equiv P\left[\nu_{4}\left(\pi_{g}\right)\right], P_{2} \equiv P\left[\nu_{5}\left(\pi_{u}\right)\right], P_{3} \equiv P\left[2 \nu_{4}\left(\sigma_{g}^{+}\right)\right], P_{4} \equiv$ $P\left[2 \nu_{4}\left(\delta_{q}\right)\right], P_{5} \equiv P\left[\nu_{4}+\nu_{5}\left(\sigma_{u}^{+}\right)\right], P_{6} \equiv P\left[\nu_{4}+\nu_{5}\left(\sigma_{u}^{-}\right)\right], P_{7} \equiv P\left[\nu_{4}+\nu_{5}\left(\delta_{u}\right)\right]$, $P_{8} \equiv P\left[2 \nu_{5}\left(\sigma_{g}^{+}\right)\right]$, and $P_{9} \equiv P\left[2 \nu_{5}\left(\delta_{g}\right)\right]$. The $\pi$ and $\delta$ levels are split into two sublevels with opposite parities, $e$ and $f$ (see Appendix A). In the figure they have been merged into a single level with degeneracy 2 . Only vibrational levels up to $2 \nu_{5}\left(\delta_{g}\right)$ have been plotted although higher energy levels have been considered in the calculations. The main box contains the vibrational levels, while the upper inset focuses on the high-energy ones. The right boxes contain $\mathrm{C}_{2} \mathrm{H}_{2}$ density (lower) and $T_{K}$ (upper). 


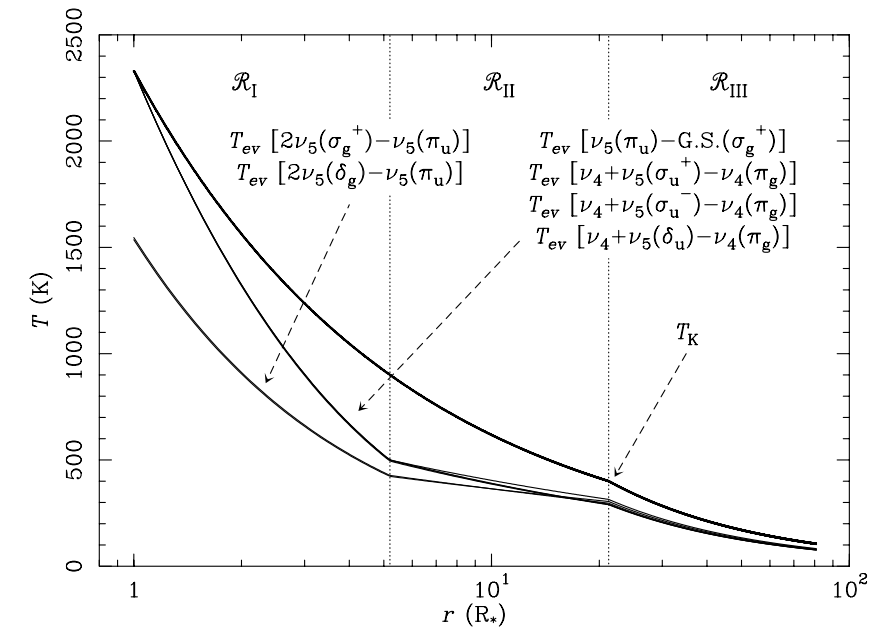

FIG. 12.-Kinetic and vibrational excitation temperatures for the observed transitions of $\mathrm{C}_{2} \mathrm{H}_{2} . T_{\mathrm{ev}}$ are merged into two different groups. In spite of the similarity of the dipole moments of the transitions, $T_{\mathrm{ev}}\left[2 \nu_{5}\left(\sigma_{g}^{+}\right) \leftrightarrow \nu_{5}\left(\pi_{u}\right)\right]$ and $T_{\mathrm{ev}}\left[2 \nu_{5}\left(\delta_{g}\right) \leftrightarrow \nu_{5}\left(\pi_{u}\right)\right]$ are quite different from $T_{\mathrm{ev}}\left[\nu_{4}+\nu_{5}\left(\sigma_{u}^{ \pm}\right) \leftrightarrow \nu_{4}\left(\pi_{g}\right)\right]$, $T_{\mathrm{ev}}\left[\nu_{4}+\nu_{5}\left(\delta_{u}\right) \leftrightarrow \nu_{4}\left(\pi_{g}\right)\right]$, and $T_{\mathrm{ev}}\left[\nu_{5}\left(\pi_{u}\right) \leftrightarrow\right.$ G.S. $\left.\left(\sigma_{g}^{+}\right)\right]$. All the considered vibrational levels are out of LTE over the whole CSE. Note that $2 \nu_{5}\left(\sigma_{g}^{+}\right)$and $2 \nu_{5}\left(\delta_{g}\right)$ are out of LTE even near the star.

slope of $P_{0}$ and $P_{1}$ falls, while it increases for the other levels. The absorption of $3 \mu \mathrm{m}$ radiation, which pumps $\mathrm{C}_{2} \mathrm{H}_{2}$ from the ground to high-energy levels, changes the slope of the population of the ground state with respect to that under LTE throughout regions I and II. This radiation also affects the $\nu_{4}\left(\pi_{g}\right)$ level, which starts to be efficiently pumped from the radiative cascade from the stretching modes. The ratio $P_{1} / P_{0}$ is larger in regions II and III than under LTE. However, as the transition $\nu_{5}\left(\pi_{u}\right) \leftrightarrow$ G.S. $\left(\sigma_{g}^{+}\right)$is strong, the ratio $P_{2} / P_{1}$ is lower than expected under LTE. All the vibrational levels above $\nu_{5}$ are underpopulated in region II. Collisions are less efficient as the volume density in this region is low and all vibrational pumping is radiatively dominated.

5. Levels $2 \nu_{4}\left(\sigma_{g}^{+}\right)$and $2 \nu_{4}\left(\delta_{g}\right)$ are metastable states like $\nu_{4}\left(\pi_{g}\right)$. On the other hand, $\nu_{5}\left(\pi_{u}\right)$ is strongly radiatively connected to the ground state. Consequently, $P_{3} / P_{2}$ is larger than it would be under LTE for $r>R_{d 1}$.

6. $P_{6} / P_{5}$ is larger in region II than expected under LTE. The populations of the vibrational levels $\nu_{4}+\nu_{5}\left(\sigma_{u}^{+}\right), \nu_{4}+\nu_{5}\left(\sigma_{u}^{-}\right)$, and $\nu_{4}+\nu_{5}\left(\delta_{u}\right)$ are similarly increased due to radiative cascades from higher energy levels. However, the strong radiative transition $\nu_{4}+\nu_{5}\left(\sigma_{u}^{+}\right) \leftrightarrow$ G.S. $\left(\sigma_{q}^{+}\right)$(Herzberg 1989b, p. 290) increases the ratio $P_{6} / P_{5}$ due to stimulated de-excitation of molecules from $\nu_{4}+\nu_{5}\left(\sigma_{u}^{+}\right)$by $7.5 \mu \mathrm{m}$ radiation. This mechanism is further strengthened due to the forbidden transition $\nu_{4}+\nu_{5}\left(\sigma_{u}^{-}\right) \leftrightarrow$ G.S. $\left(\sigma_{g}^{+}\right)$.

7. $P_{4} / P_{3}, P_{5} / P_{4}$, and $P_{7} / P_{6}$ are consistent with LTE as these levels are separated by an energy equivalent to $4.5,136.1$, and $10.9 \mathrm{~K}$, respectively. As levels $\nu_{4}+\nu_{5}\left(\sigma_{u}^{-}\right)$and $\nu_{4}+\nu_{5}\left(\delta_{u}\right)$ are not radiatively connected to the ground state, the collisions keep $P_{7} \simeq 2 P_{6}$ (see Fig. 11). There may be a slight departure from LTE for the ratio $P_{5} / P_{4}$ in region III $\left[T_{K}\left(100 R_{*}\right) \simeq 85 \mathrm{~K}\right]$, although it cannot be derived from the observational data.

8 . For distances larger than $20 R_{*}$ (region III), only the lower energy levels are significantly populated. The most intense transition in the outer envelope is $\nu_{5}\left(\pi_{u}\right) \leftrightarrow$ G.S. $\left(\sigma_{g}^{+}\right)$. Farther away from the star, it is not possible to find any emission from $\mathrm{C}_{2} \mathrm{H}_{2}$.

9. As for region II, $P_{1} / P_{0}$ in region III is larger than it would be under LTE, but it decreases rapidly, as in the LTE case. This comes about from absorption of MIR radiation in region III. This radiation excites molecules from the state $\nu_{4}$ toward $\nu_{4}+\nu_{5}$, and then the molecule de-excites to the ground state. The $3 \mu \mathrm{m}$ pumping mechanism, important in region II, loses its effectiveness at distances larger than $R_{d 2}$ due to the decreasing intensity of the NIR radiation. In addition, since the gas density and $T_{K}$ are low, collisions are ineffective in de-exciting molecules from level $\nu_{4}\left(\pi_{g}\right)$. Furthermore, no molecules are in that state in the outer CSE.

10. $P_{9} / P_{8}$ is consistent with LTE in region I but shows a departure from LTE in regions II and III. Level $2 \nu_{5}\left(\sigma_{q}^{+}\right)$is radiatively connected to the ground state through $2 \nu_{5}\left(\sigma_{g}^{+}\right) \leftrightarrow \nu_{2}+\nu_{4}+$ $\nu_{5}\left(\sigma_{u}^{+}\right) \leftrightarrow$ G.S. $\left(\sigma_{g}^{+}\right)$and $2 \nu_{5}\left(\sigma_{g}^{+}\right) \leftrightarrow \nu_{5}\left(\pi_{u}\right) \leftrightarrow$ G.S. $\left(\sigma_{g}^{+}\right)$. On the contrary, $2 \nu_{5}\left(\delta_{g}\right)$ is connected to the ground state via the transition $2 \nu_{5}\left(\delta_{g}\right) \leftrightarrow \nu_{5}\left(\pi_{u}\right) \leftrightarrow$ G.S. $\left(\sigma_{g}^{+}\right)$but not through $2 \nu_{5}\left(\delta_{g}\right) \leftrightarrow \nu_{2}+$ $\nu_{4}+\nu_{5}\left(\delta_{u}\right) \leftrightarrow$ G.S. $\left(\sigma_{g}^{+}\right)$because $\nu_{2}+\nu_{4}+\nu_{5}\left(\delta_{u}\right) \leftrightarrow$ G.S. $\left(\sigma_{g}^{+}\right)$ is forbidden. Therefore, when collisional pumping loses its effectiveness $\left(r>R_{d 1}\right), P_{9} / P_{8}$ becomes lower than under LTE.

The estimation of the populations in the $P_{3}$ and $P_{4}$ levels is subject to large uncertainties because the observed lines arising from these levels are weak. We believe that even for $P_{3}$ and $P_{4}$ the obtained vibrational temperatures are good estimators (see Fig. 11). The rest of the vibrational populations are well determined based on the observational data.

\subsection{Abundances}

The condensation of refractory molecules on dust grains over the two acceleration zones modifies the abundances of several molecular species. It is unclear to what degree $\mathrm{C}_{2} \mathrm{H}_{2}$ (and isotopologs) condenses onto dust grains, but our data provide a tool to check whether or not significant changes occur from one region to another.

As we show below ( large uncertainties due to their small influence on most observed lines compared with the rest of the CSE. The angular size of the innermost region is small $\left(\simeq 0.2^{\prime \prime}\right)$, and despite the high values of density and $T_{K}$, the observed flux is small with respect to that coming from outer shells of the envelope. On the other hand, the efficiency of a PSF $\simeq 1^{\prime \prime}$ is significant for the angular size of region II $\left(\simeq 0.8^{\prime \prime}\right)$. Loss of light at the spectrograph entrance slit, which is $\simeq 1.6^{\prime \prime}$ wide, is still relatively unimportant for region II but is a large fraction of the radiation emitted in region III. In addition, $T_{K}$ and the density are still high in region II (the mean $T_{K}$ and $n$ for $\mathrm{C}_{2} \mathrm{H}_{2}$ are $\simeq 560 \mathrm{~K}$ and $220 \mathrm{~cm}^{-3}$, respectively). Therefore, most of the emission for optically thick lines (extending farther out than $R_{d 1}$ ) and the bulk absorption for thin ones (produced in region I) are due to region II. In region III, $T_{K}$ and the density are low so emission from this region is anticipated to be small. Furthermore, region III suffers the most from light loss at the slit, which pertains only to the emission component of the thick line profiles. Consequently, region III need only be considered for the very thick lines [e.g., rovibrational lines of the vibrational transition $\nu_{5}\left(\pi_{u}\right) \leftrightarrow$ G.S. $\left.\left(\sigma_{g}^{+}\right)\right]$, for which the bulk absorption is produced by this region. This implies that the maximum information on the $\mathrm{C}_{2} \mathrm{H}_{2}$ abundance in region III can be obtained from absorption in the P Cygni profiles of these thick lines. Unfortunately, their thickness precludes the possibility of getting accurate information on the abundances of $\mathrm{C}_{2} \mathrm{H}_{2}$ at angles larger than $1^{\prime \prime}\left(r>50 R_{*}\right)$. At larger radii, the absorption component of the lines is almost completely saturated. At distances larger than $300 R_{*}$, there is no emission from $\mathrm{C}_{2} \mathrm{H}_{2}$ because all the molecules remain in the ground state (see Fig. 11) and pure rotational transitions are forbidden due to the lack of electric dipole moment. 
TABLE 5

Vibrational Temperatures of $\mathrm{H}^{13} \mathrm{CCH}$

\begin{tabular}{|c|c|c|c|c|c|c|c|}
\hline Vibrational Level & $P_{i}$ & $\begin{array}{c}T_{1} \\
(\mathrm{~K})\end{array}$ & $\alpha_{1}$ & $\begin{array}{c}T_{2} \\
(\mathrm{~K})\end{array}$ & $\alpha_{2}$ & $\begin{array}{c}T_{3} \\
(\mathrm{~K})\end{array}$ & $\alpha_{3}$ \\
\hline G.S. .. & $P_{0}$ & 2330 & 0.58 & 900 & 0.56 & 410 & 1.00 \\
\hline$\nu_{4}(\pi)$. & $P_{1}$ & 2330 & 0.58 & 900 & 1.92 & 60 & 1.00 \\
\hline$\nu_{5}(\pi) \ldots \ldots \ldots \ldots \ldots \ldots \ldots \ldots \ldots \ldots \ldots \ldots$ & $P_{2}$ & 2330 & 0.58 & 900 & 1.43 & 120 & 1.00 \\
\hline $2 \nu_{4}\left(\sigma^{+}\right) \ldots \ldots \ldots \ldots \ldots \ldots \ldots \ldots \ldots \ldots \ldots$ & $P_{3}$ & 2330 & 0.58 & 900 & 0.58 & 400 & 1.00 \\
\hline $2 \nu_{4}(\delta) \ldots \ldots \ldots \ldots \ldots \ldots \ldots \ldots$ & $P_{4}$ & 2330 & 0.58 & 900 & 0.58 & 400 & 1.00 \\
\hline$\nu_{4}+\nu_{5}\left(\sigma^{+}\right) \ldots \ldots \ldots \ldots \ldots \ldots$ & $P_{5}$ & 2330 & 0.58 & 900 & 0.58 & 400 & 1.00 \\
\hline$\nu_{4}+\nu_{5}\left(\sigma^{-}\right) \ldots \ldots \ldots \ldots \ldots \ldots$ & $P_{6}$ & 2330 & 0.58 & 900 & 0.58 & 400 & 1.00 \\
\hline$\nu_{4}+\nu_{5}(\delta) \ldots \ldots \ldots \ldots \ldots \ldots \ldots \ldots \ldots \ldots$ & $P_{7}$ & 2330 & 0.93 & 500 & 2.78 & 10 & 1.00 \\
\hline $2 \nu_{5}\left(\sigma^{+}\right) \ldots \ldots \ldots \ldots \ldots \ldots \ldots \ldots \ldots \ldots \ldots$ & $P_{8}$ & 2330 & 0.58 & 900 & 0.58 & 400 & 1.00 \\
\hline$T_{K}$ & $\ldots$ & 2330 & 0.58 & 900 & 0.58 & 400 & 1.00 \\
\hline
\end{tabular}

Notes.-Vibrational temperatures of $\mathrm{H}^{13} \mathrm{CCH}$. See Table 4 for details about the meaning of the constants.

The $\mathrm{C}_{2} \mathrm{H}_{2}$ abundances derived from the best fits for each region are

$$
x_{\mathrm{C}_{2} \mathrm{H}_{2}}(r)= \begin{cases}7.5 \times 10^{-6}, & \text { region } \mathrm{I}, \\ 8.0 \times 10^{-5}, & \text { region } \mathrm{II}, \\ 8.0 \times 10^{-5}, & \text { region } \mathrm{III},\end{cases}
$$

with an assumed initial value of $8 \times 10^{-5}$ for the CSE. The derived column density is $\simeq 1.6 \times 10^{19} \mathrm{~cm}^{-2}$.

Note that the abundance in region I is an order of magnitude lower than in the rest of the CSE. A higher abundance in that region implies diminishing its size, incompatible with previous results (see $\S 4$ ). We discuss this topic further in $\S 8$. The abundances of $\mathrm{C}_{2} \mathrm{H}_{2}$ in regions II and III are similar to those obtained in previous studies: $8 \times 10^{-5}$ and $5 \times 10^{-5}$ from Keady \& Ridgway (1993) and Cernicharo et al. (1999), respectively.

In addition, the increment in the $\mathrm{C}_{2} \mathrm{H}_{2}$ abundance between regions I and II is compatible with that yielded by LTE calculations (M. Agúndez 2006, private communication) and with the results of the chemistry in a pulsating star published by Cherchneff (2006). The variation in the LTE abundance profile between both regions ranges from $15 \%$ to $99 \%$ (with respect to the abundance in region II), depending on the physical conditions of the very inner envelope (Agúndez \& Cernicharo 2006). The action of shocks on chemical abundances at radii smaller than $5 R_{*}$ produces abundance profiles that increase by $99 \%$ the abundance in region II (Cherchneff 2006). Both models could explain the increment of $90 \%$ derived in this work. We also discuss this topic in $\S 8$.

The uncertainties on the abundances (see Table 7) are compatible with a small condensation of $\mathrm{C}_{2} \mathrm{H}_{2}$ (and isotopologs) on dust grains and the consequent existence of an acceleration regime between regions II and III. Unfortunately, we cannot assure the existence of these processes with the data derived.

\section{6. $\mathrm{H}^{13} \mathrm{CCH}$ AND ${ }^{13} \mathrm{C}_{2} \mathrm{H}_{2}$ MODELING}

As in the case of $\mathrm{C}_{2} \mathrm{H}_{2}$, we can derive the abundance and vibrational temperatures and constrain the parameters related to the physical conditions of the CSE, through fitting as many $\mathrm{H}^{13} \mathrm{CCH}$ lines as possible. The fitting procedure is the same as for $\mathrm{C}_{2} \mathrm{H}_{2}$ (see $\S 5$ ). We have used the physical conditions determined previously with $\mathrm{C}_{2} \mathrm{H}_{2}$ as initial inputs for the fits of the $\mathrm{H}^{13} \mathrm{CCH}$ lines.

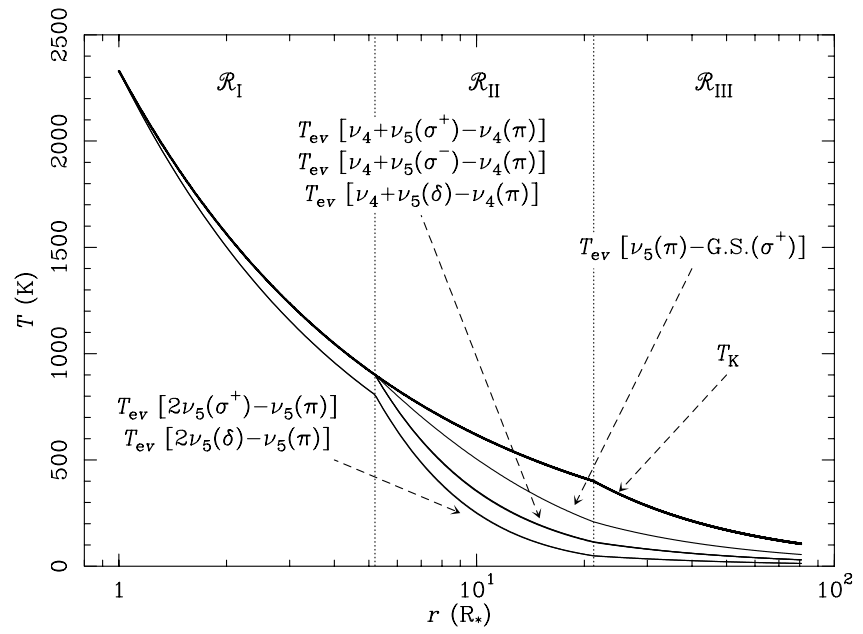

FIG. 13.-Kinetic and vibrational excitation temperatures for the $\mathrm{H}^{13} \mathrm{CCH}$ observed transitions. As for $\mathrm{C}_{2} \mathrm{H}_{2}, T_{\mathrm{ev}}$ are merged into two different groups, although in this case, the transition $\nu_{5}(\pi) \leftrightarrow$ G.S. $\left(\sigma^{+}\right)$stands alone with a $T_{\text {ev }}$ not much lower than $T_{K}$. All the selected transitions are under LTE in region I.

\subsection{Abundances}

The $\left[\mathrm{C}_{2} \mathrm{H}_{2}\right] /\left[\mathrm{H}^{13} \mathrm{CCH}\right]$ ratio is easily determined taking into account that the abundance of $\mathrm{H}^{13} \mathrm{CCH}$ must be proportional to that of $\mathrm{C}_{2} \mathrm{H}_{2}$ :

$$
\frac{\left[\mathrm{C}_{2} \mathrm{H}_{2}\right]}{\left[\mathrm{H}^{13} \mathrm{CCH}\right]} \simeq \frac{1}{0.049} \simeq 20.5,
$$

where the fitted lines are shown in Table 2 . This value is very similar to $\left[\mathrm{C}_{2} \mathrm{H}_{2}\right] /\left[\mathrm{H}^{13} \mathrm{CCH}\right] \simeq 22$, proposed by Cernicharo et al. $(1996,1999,2000)$. Hence, the abundance ratio $\left[{ }^{12} \mathrm{C}\right] /\left[{ }^{13} \mathrm{C}\right] \mathrm{de}-$ rived from our data is $\simeq 41$ in $\mathrm{IRC}+10216$.

\subsection{Vibrational Temperatures}

As shown in Appendices $\mathrm{A}$ and $\mathrm{B}$, the spectrum of $\mathrm{H}^{13} \mathrm{CCH}$ presents some differences with respect to $\mathrm{C}_{2} \mathrm{H}_{2}$ [e.g., $\nu_{4}(\pi)-$ G.S. $\left(\sigma^{+}\right)$is IR active for $\mathrm{H}^{13} \mathrm{CCH}$ while it remains forbidden for $\mathrm{C}_{2} \mathrm{H}_{2}$ ]. Consequently, although $\mathrm{H}^{13} \mathrm{CCH}$ is very similar to $\mathrm{C}_{2} \mathrm{H}_{2}$, its vibrational temperatures are different (see Table 5 and Fig. 13). The observed $\mathrm{H}^{13} \mathrm{CCH}$ transitions also support the NIR pumping mechanism proposed in $\S 5.5$. For $\mathrm{H}^{13} \mathrm{CCH}$, more transitions are allowed than for $\mathrm{C}_{2} \mathrm{H}_{2}$ due to combined levels involving both stretching and bending modes. These differences can be understood in terms of the radiative pumping paths for both isotopologs (see below):

1. In region I, the population of $2 \nu_{5}\left(\sigma^{+}\right)$is lower than in LTE compared with that of $\nu_{4}+\nu_{5}(\delta)$. Radiative cascades from higher energy levels populate these states in a different manner than in the $\mathrm{C}_{2} \mathrm{H}_{2}$ case (e.g., $\nu_{3}+\nu_{5} \leftrightarrow 2 \nu_{5}$ is allowed for $\mathrm{H}^{13} \mathrm{CCH}$ ), keeping level $2 \nu_{5}\left(\sigma^{+}\right)$almost in LTE next to the photosphere (see $\left.\S 5.5\right)$. Therefore, all the vibrational levels can be considered in LTE close to the star (see Fig. 13).

2. The allowed transitions for $\mathrm{H}^{13} \mathrm{CCH}$ connecting $2 \nu_{5}$ to lower energy levels and the increase of the population of $\nu_{5}$ through $\nu_{4}+\nu_{5} \leftrightarrow \nu_{5}$ result in a decrease of $T_{\text {ev }}\left(2 \nu_{5} \leftrightarrow \nu_{5}\right)$ in regions II and III, with respect to the $\mathrm{C}_{2} \mathrm{H}_{2}$ case.

3. In regions II and III, $P_{2} / P_{1}, P_{3} / P_{2}$, and $P_{8} / P_{7}$ are lower than in LTE, as for the $\mathrm{C}_{2} \mathrm{H}_{2}$ case. However, several radiative transitions in $\mathrm{H}^{13} \mathrm{CCH}$, which are forbidden for $\mathrm{C}_{2} \mathrm{H}_{2}$, keep these ratios closer to LTE. 
4. The $\nu_{4}\left(\pi_{q}\right)$ vibrational level in $\mathrm{C}_{2} \mathrm{H}_{2}$ is only collisionally connected to the ground state, while it is connected both collisionally and radiatively in $\mathrm{H}^{13} \mathrm{CCH}$. This fact reduces the ratio $P_{1} / P_{0}$ and thermalizes the $\nu_{4}$ state over almost the whole CSE. In addition, $P_{2} / P_{1}$ is in LTE in region I, where collisions can thermalize the $\nu_{5}$ state, but it is out of LTE in regions II and III once $T_{K}$ and the density have decreased and radiative excitation has become the main pumping mechanism. As the transition $\nu_{4} \leftrightarrow$ G.S. is weaker than $\nu_{5} \leftrightarrow$ G.S. (Di Lonardo et al. 2002), we can conclude that pumping of molecules from the ground state to $\nu_{4}$ and $\nu_{5}$ through radiative cascades from high-energy vibrational levels seems to be almost the same (see Fig. 10).

5. $P_{3} / P_{2}$ is lower than in LTE in regions II and III for several reasons: $2 \nu_{4}\left(\sigma^{+}\right)$is radiatively connected to $\nu_{4}(\pi)$ and G.S. $\left(\sigma^{+}\right)$, molecules are pumped from the ground state to $\nu_{5}(\pi)$ through radiative cascades, and collisional pumping is not effective enough to keep the vibrational populations under LTE in these regions.

6. As for $\mathrm{C}_{2} \mathrm{H}_{2}$, the model points out that $P_{4} / P_{3}, P_{5} / P_{4}$, and $P_{7} / P_{6}$ can be considered to be in LTE.

7. $T_{\mathrm{ev}}\left[\nu_{5}(\pi) \leftrightarrow\right.$ G.S. $\left.\left(\sigma^{+}\right)\right]<T_{K}$ because the transition is strong. On the other hand, the transition $\nu_{4}+\nu_{5} \leftrightarrow \nu_{5}$ could depopulate $\nu_{4}+\nu_{5}$, which is compatible with $T_{\mathrm{ev}}\left(\nu_{4}+\nu_{5} \leftrightarrow \nu_{4}\right)<$ $T_{\text {ev }}\left(\nu_{5} \leftrightarrow\right.$ G.S. $)$, in contrast to the behavior of these temperatures for $\mathrm{C}_{2} \mathrm{H}_{2}$, where they are equal.

\subsection{Rotational Temperatures}

Unlike $\mathrm{C}_{2} \mathrm{H}_{2}$, all $\mathrm{H}^{13} \mathrm{CCH}$ chosen lines can be fitted quite well with the rotational levels in LTE. No ad hoc rotational temperatures are needed.

\subsection{Searching for ${ }^{13} \mathrm{C}_{2} \mathrm{H}_{2}$}

Given the large abundance of $\mathrm{C}_{2} \mathrm{H}_{2}$, detection of ${ }^{13} \mathrm{C}_{2} \mathrm{H}_{2}$ rovibrational lines may be possible. The double isotopic substitution of the $\mathrm{C}$ atoms greatly reduces the abundance of this species compared with $\mathrm{C}_{2} \mathrm{H}_{2}$. As we have derived in $\S 6,\left[{ }^{12} \mathrm{C}\right] /\left[{ }^{13} \mathrm{C}\right] \simeq 41$. Consequently, $\left[{ }^{13} \mathrm{C}_{2} \mathrm{H}_{2}\right] /\left[\mathrm{C}_{2} \mathrm{H}_{2}\right] \simeq(1 / 41)^{2} \simeq 6 \times 10^{-4}$. Unfortunately, the strongest bands of ${ }^{13} \mathrm{C}_{2} \mathrm{H}_{2}$ are in a range of the spectrum crowded with strong $\mathrm{C}_{2} \mathrm{H}_{2}, \mathrm{H}^{13} \mathrm{CCH}$, and $\mathrm{HCN}$ transitions (see Fig. 1). We have compared the optical depth of one of the strongest ${ }^{13} \mathrm{C}_{2} \mathrm{H}_{2}$ lines, $\nu_{5}\left(\pi_{u}\right) R_{e}(3)$, with the same line for $\mathrm{C}_{2} \mathrm{H}_{2}$. The dipole moment employed in the calculations for ${ }^{13} \mathrm{C}_{2} \mathrm{H}_{2}$ has been set equal to that of $\mathrm{C}_{2} \mathrm{H}_{2}$ due to the lack of data on intensities quoted in the literature. The optical depth of this line has been computed by running the code with the parameters derived from the fits to the observed $\mathrm{C}_{2} \mathrm{H}_{2}$ lines and using the doublesubstitution isotopic ratio given above. The derived optical depths along the line of sight, without considering the Doppler effect, are 630 for $\mathrm{C}_{2} \mathrm{H}_{2}$ and 0.033 for ${ }^{13} \mathrm{C}_{2} \mathrm{H}_{2}$. The low optical depth for the ${ }^{13} \mathrm{C}_{2} \mathrm{H}_{2}$ line results in a maximum absorption $<1 \%$ of the continuum flux and cannot be observed in our data. An equal or smaller absorption is expected for other lines of the same band.

\section{7. $\mathrm{HCN}$ AND $\mathrm{H}^{13} \mathrm{CN}$ MODELING}

Modeling $\mathrm{HCN}$ (and $\mathrm{H}^{13} \mathrm{CN}$ ) lines adds additional constraints to the physical conditions over the CSE for $r<100 R_{*}$. The spectra shown in Figures 1-6 indicate the presence of many rovibrational lines from the $\nu_{2}$ bending mode and several associated overtones. As for $\mathrm{C}_{2} \mathrm{H}_{2}$ and $\mathrm{H}^{13} \mathrm{CCH}$, we have selected a sample of $\mathrm{HCN}$ ( and $\mathrm{H}^{13} \mathrm{CN}$ ) lines to fit to the data and to derive physical and chemical conditions through the CSE (see some fits in Fig. 14). The observation of transitions arising from overtones and $\mathrm{H}^{13} \mathrm{CN}$ allows us to study optically thinner lines compared to those of the fundamental bending mode.

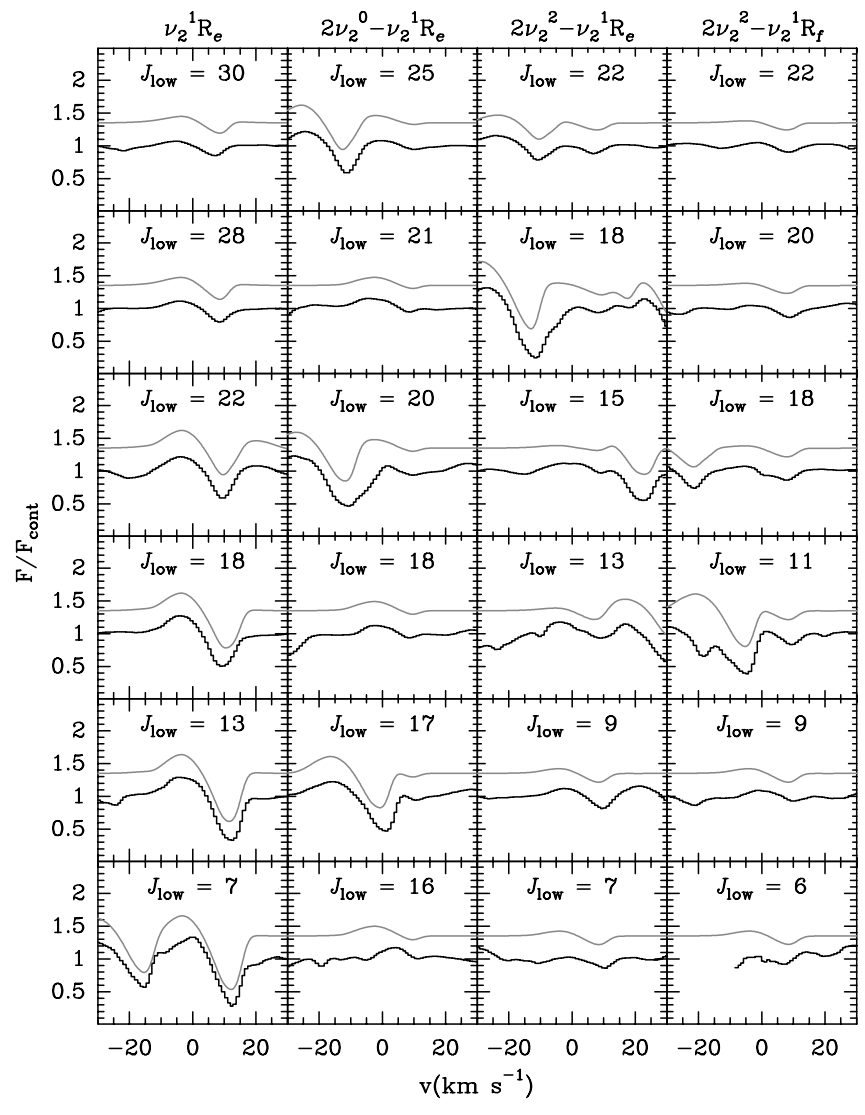

FIG. 14.-Fits of some lines of HCN. The black lines are the observed spectra, while the gray lines are the fits. The data have been corrected from observational frequency deviations adding/suppressing the mean difference between the observational and the laboratory frequency of many lines (see $\S 2$ ). The existing discrepancies are small and can be assumed as blends with other lines and/or observational errors (the spectroscopic notation is described in Appendix A). [See the electronic edition of the Journal for a color version of this figure.]

The differences between $\mathrm{H}^{13} \mathrm{CN}$ and the main isotopolog, $\mathrm{HCN}$, lead to a significant increment in the molecular mass and a slight variation in the interatomic distances. Consequently, the wave functions of each molecular state and their related energies are not equal for $\mathrm{HCN}$ and $\mathrm{H}^{13} \mathrm{CN}$. Although some spectroscopic work on $\mathrm{H}^{13} \mathrm{CN}$ can be found in the literature, there are no data available about the intensities of its transitions, while HCN has been studied more extensively. Therefore, we have used the dipole moments of $\mathrm{HCN}$ for $\mathrm{H}^{13} \mathrm{CN}$ as a reasonable approximation for the transition strengths.

The number of lines used for the fits is shown in Table 2. Information about frequencies, intensities, and nomenclature for $\mathrm{HCN}$ and its isotopologs can be found in Appendices A and B and references therein.

\subsection{Vibrational Temperatures}

As seen in previous sections, $\mathrm{C}_{2} \mathrm{H}_{2}$ does not present any permanent dipole moment (it is very small for $\mathrm{H}^{13} \mathrm{CCH}$ and can be neglected to a first approximation). Considering rotational LTE as suitable initial input, there will not be any pure rotational emission. Fortunately, in spite of the permanent dipole moment, $\mathrm{HCN}$ (and $\mathrm{H}^{13} \mathrm{CN}$ ) seems to be under LTE for low- $J$ rotational levels, making easier the determination of the abundance and $T_{\text {vib }}$ (see $\S 7.2)$.

Near $R_{d 1}, T_{d} \simeq 800-850 \mathrm{~K}$ and the dust blackbody emission peaks at $\simeq 3.5 \mu \mathrm{m}\left(\simeq 2850 \mathrm{~cm}^{-1}\right)$. This radiation pumps HCN from the ground state to the stretching and combination levels $\nu_{3}\left(\sigma^{+}\right)$, 


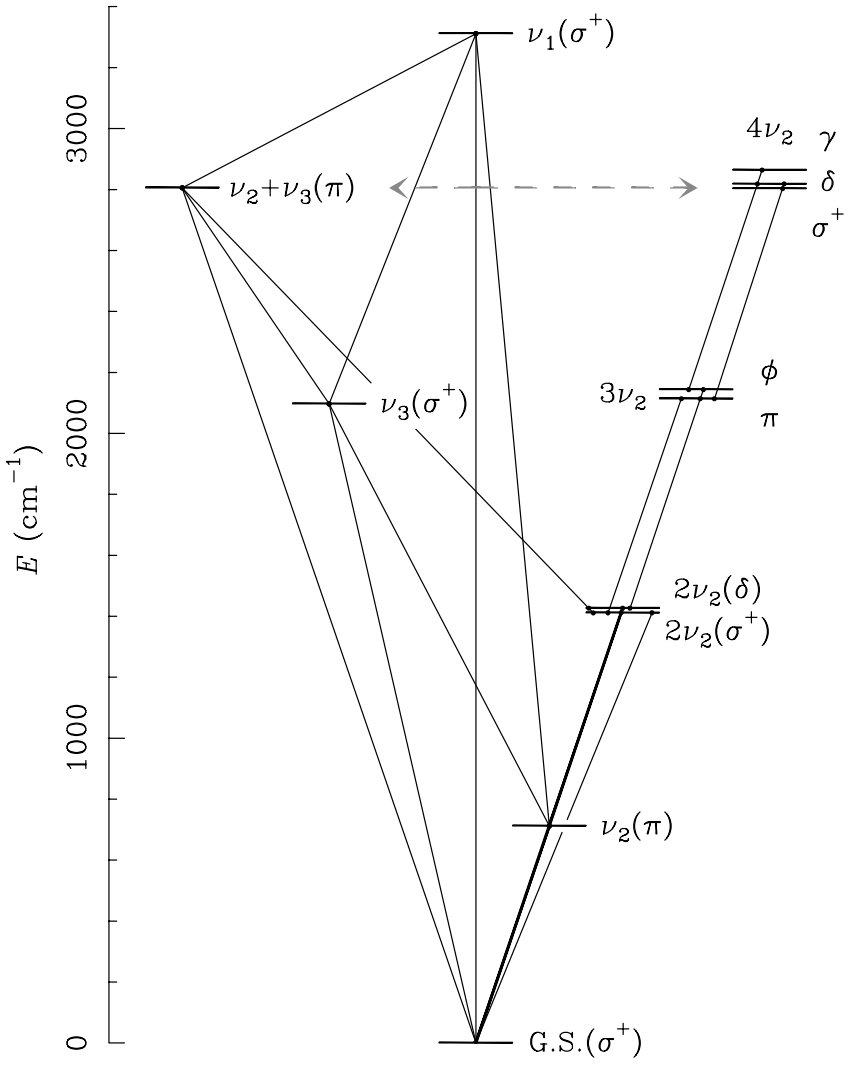

FIG. 15.-Energy of the vibrational levels of HCN. This pattern is the same for the $\mathrm{H}^{13} \mathrm{CN}$ isotopolog. The transitions given are the perpendicular ones with $\Delta v_{2}= \pm 1$ and the most intense parallel ones. The thin lines are allowed transitions. The thick lines are observed vibrational transitions. The gray dashed double arrow indicates the resonance between several rotational levels of the vibrational states $\nu_{2}+\nu_{3}(\pi)$ and $4 \nu_{2}\left(\sigma^{+}\right)$. [See the electronic edition of the Journal for a color version of this figure.]

$\nu_{2}+\nu_{3}(\pi)$, and $\nu_{1}\left(\sigma^{+}\right)$, with energies 2096.85, 2807.05, and $3311.48 \mathrm{~cm}^{-1}$, respectively (see Fig. 15 ). In addition, several rotational levels of $4 \nu_{2}\left(\sigma^{+}\right)$(with energy $2802.96 \mathrm{~cm}^{-1}$ ) are strongly connected to the ground state through a resonance with the corresponding rotational levels of $\nu_{2}+\nu_{3}(\pi)$. This resonance could explain the HCN masers observed by Schilke \& Menten (2003) in the $4 \nu_{2}\left(\sigma^{+}\right)$state. Radiative de-excitation changes the population of mode $\nu_{2}(\pi)$ and its overtones with respect to the LTE case.

The vibrational temperatures derived from the fits are presented in Table 6 and plotted in Figure 16. As in the case of $\mathrm{C}_{2} \mathrm{H}_{2}$, we have labeled the populations of the levels as follows: $P_{0} \equiv P\left[\right.$ G.S. $\left.\left(\sigma^{+}\right)\right]$, $P_{1} \equiv P\left[\nu_{2}(\pi)\right], P_{2} \equiv P\left[2 \nu_{2}\left(\sigma^{+}\right)\right]$, and $P_{3} \equiv P\left[2 \nu_{2}(\delta)\right]$.

1. $T_{\mathrm{vib}}\left[\nu_{2}(\pi) \leftrightarrow\right.$ G.S. $\left.\left(\sigma^{+}\right)\right] \simeq T_{K}$ in region $\mathrm{I}$, departing from $T_{K}$ in regions II and III. In region I, collisions thermalize $\nu_{2}(\pi)$,

TABLE 6

Vibrational Temperatures of $\mathrm{HCN}$ and $\mathrm{H}^{13} \mathrm{CN}$

\begin{tabular}{|c|c|c|c|c|c|c|c|}
\hline Vibrational Level & $P_{i}$ & $\begin{array}{c}T_{1} \\
(\mathrm{~K})\end{array}$ & $\alpha_{1}$ & $\begin{array}{c}T_{2} \\
(\mathrm{~K})\end{array}$ & $\alpha_{2}$ & $\begin{array}{c}T_{3} \\
(\mathrm{~K})\end{array}$ & $\alpha_{3}$ \\
\hline G.S. ......................... & $P_{0}$ & 2330 & 0.58 & 900 & 1.56 & 130 & 1.00 \\
\hline$\nu_{2}(\pi) \ldots \ldots \ldots \ldots \ldots \ldots \ldots \ldots \ldots$ & $P_{1}$ & 1250 & 0.82 & 600 & 0.62 & 250 & 1.00 \\
\hline $2 \nu_{2}\left(\sigma^{+}\right) \ldots \ldots \ldots \ldots \ldots \ldots \ldots \ldots \ldots$ & $P_{2}$ & 800 & 1.26 & 100 & 1.24 & 17.5 & 1.00 \\
\hline$T_{K} \ldots \ldots \ldots \ldots \ldots \ldots \ldots \ldots \ldots \ldots \ldots \ldots \ldots \ldots$ & $\ldots$ & 2330 & 0.58 & 900 & 0.58 & 400 & 1.00 \\
\hline
\end{tabular}

NoTES.-Vibrational temperatures of $\mathrm{HCN}$ and $\mathrm{H}^{13} \mathrm{CN}$. See Table 4 for details about the meaning of the constants. The parameters of the vibrational temperatures of $\mathrm{H}^{13} \mathrm{CN}$ are only those related to the ground state.

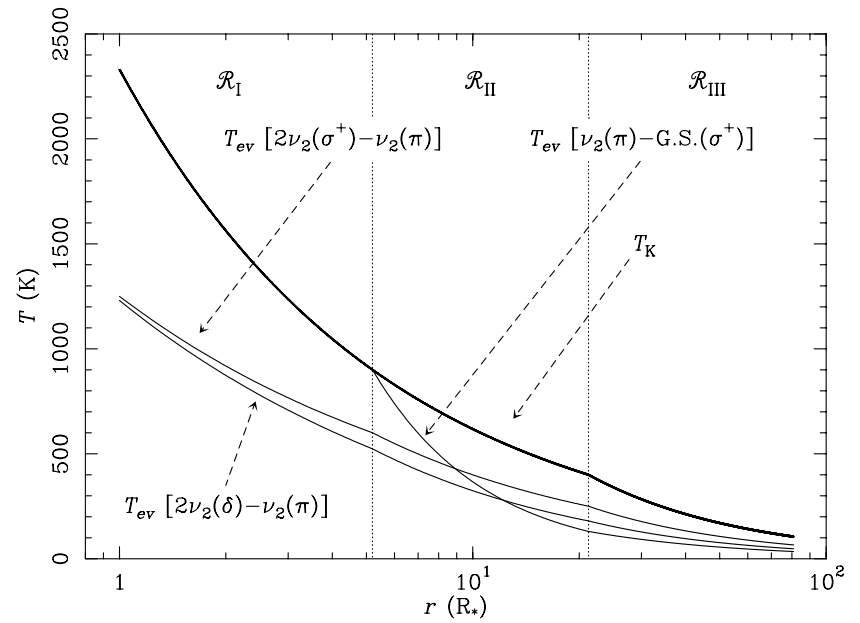

FIG. 16.-Kinetic and vibrational excitation temperatures for $\mathrm{HCN}$ observed transitions. See text for details.

while at larger distances only infrared photons play a role in the pumping. Vibrational levels $\nu_{1}\left(\sigma^{+}\right)$and $\nu_{3}\left(\sigma^{+}\right)$are radiatively connected to the bending level $\nu_{2}(\pi)$. In addition, $\nu_{2}(\pi)$ can also be populated through the loop G.S. $\left(\sigma^{+}\right) \rightarrow \nu_{2}+\nu_{3}(\pi) \rightarrow \nu_{3}\left(\sigma^{+}\right) \rightarrow$ $\nu_{2}(\pi)$. The band $\nu_{2}(\pi)-$ G.S. $\left(\sigma^{+}\right)$is quite strong and the deexcitation rate is high.

2. Levels $2 \nu_{2}\left(\sigma^{+}\right)$and $2 \nu_{2}(\delta)$ are out of LTE over the whole $\mathrm{CSE}$, as well as close to the stellar photosphere. The dipole moment of the transitions involving these levels is so high that they are not thermalized even in the dense warm innermost region. Changing $T_{\text {vib }}$ of these levels at the stellar surface from 1250 to $2330 \mathrm{~K}$ produces a change of $20 \%-30 \%$ in the emission of $2 \nu_{2}\left(\sigma^{+}\right) \leftrightarrow \nu_{2}(\pi)$ rovibrational transitions. Hence, our data are very sensitive to the value of $T_{\text {vib. }}$ Level $2 \nu_{2}\left(\sigma^{+}\right)$can be pumped from the ground state and from $\nu_{2}+\nu_{3}\left(\sigma^{+}\right)$. However, the $2 \nu_{2}(\delta)$ level is not connected to the ground state except through the radiative cascades from higher energy levels (see Cernicharo et al. 1999). Therefore, $P_{3} / 2 P_{2}$ is always lower than $2 P_{2} / P_{1}$ [we add the factor of 2 to account for the difference in the degeneracy between levels $2 \nu_{2}\left(\sigma^{+}\right)$and $2 \nu_{2}(\delta)$ and between $2 \nu_{2}\left(\sigma^{+}\right)$and $\left.\nu_{2}(\pi)\right]$.

3. $T_{\mathrm{vib}}\left(\nu_{2}-\right.$ G.S. $)$ and $T_{\mathrm{vib}}\left(2 \nu_{2}-\nu_{2}\right)$ are equal to each other at $\simeq 10 R_{*}$ (see Fig. 16). Outside of the region where the NIR radiation emitted by the dust arises, the pumping via higher energy levels from the ground state (through $7 \mu \mathrm{m}$ radiation, which is the wavelength corresponding to the maximum emission of a blackbody at $T_{\mathrm{bb}} \simeq 400 \mathrm{~K}$ ) becomes less important, meaning that the population of level $2 \nu_{2}\left(\sigma^{+}\right)$increases with respect to $\nu_{2}(\pi)$.

\subsection{Rotational Temperatures}

The rotational structure of the ground state seems to be in LTE for rotational levels up to $J=20$. It is necessary to adopt ad hoc rotational temperatures for rotational levels $J=20$ and 24, when fitting the $\nu_{2} R_{e}$ lines (see Table 3). The line $\nu_{2}(\pi)-$ G.S. $\left(\sigma^{+}\right) R_{e}(21)$ can be fitted more accurately with an ad hoc $T_{\text {rot }}$ at $r=R_{d 2}$ for $J=20$ (although the fit is relatively good even under LTE). However, this ad hoc temperature is necessary to fit higher $J$ rovibrational lines. $J=24$ must be out of LTE. Under LTE, the absorption and the emission of the line $\nu_{2}(\pi)-$ G.S. $\left(\sigma^{+}\right) R_{e}(25)$ are $15 \%$ and $70 \%$ larger, respectively, compared with the observed feature. In addition, considering that this level departs from LTE with the same smooth dependence as adjacent levels, the fit is not good as the synthetic profile presents $77 \%$ more emission and $6 \%$ more absorption than the best fit. Using an ad 
hoc rotational temperature improves the fits to higher $J$ rovibrational lines. The fits suggest a quadratic deviation from LTE for the rotational pattern of the ground state beginning at $J=20$ with a decrease per rotational level of $96-7.8(J-20) \mathrm{K}$ at $r=R_{d 1}$ and of $46-3.2(J-20) \mathrm{K}$ at $r=R_{d 2}$. From the fits of bands $\nu_{2}(\pi)-$ G.S. $\left(\sigma^{+}\right)$and $2 \nu_{2}\left(\sigma^{+}\right)-\nu_{2}(\pi)$, we can assume that $\nu_{2}(\pi)$ is in LTE for the rotational levels up to $J=22$, departing smoothly from LTE for higher levels. The most important ad hoc temperature corresponds to $J=25$, being necessary to fit rovibrational lines involving higher $J$ levels. We can assume that $2 \nu_{2}\left(\sigma^{+}\right)$rotational levels are in LTE for $J \leq 26$, with it necessary to add an ad hoc rotational temperature for $J=27$. Nevertheless, fitting the bands $2 \nu_{2}(\delta)-\nu_{2}(\pi)$ also requires anomalous nonLTE rotational temperatures at the photosphere $R_{d 1}$, and $R_{d 2}$ for lines $J=12$ and 26 . The best fits to all the lines involving anomalous rotational temperatures require $T_{\text {rot }}\left(R_{d 2}\right) \leq 50 \mathrm{~K}$, although temperatures below $100 \mathrm{~K}$ are acceptable despite poorer fits.

\subsection{Abundances}

Low- $J \mathrm{HCN}$ lines of band $\nu_{2}(\pi)-\mathrm{G} . \mathrm{S}\left(\sigma^{+}\right)$are optically thick due to the large dipole moment of the vibrational transition. Many lines are saturated, and little information can be obtained from them. In particular, the abundance is a parameter that cannot be accurately determined through fits of low-energy HCN lines. The intensity of the G.S. $\left(\sigma^{+}\right)$pure rotational lines is directly related to the abundance of $\mathrm{HCN}$, while the intensity of rovibrational lines also depends on $T_{\mathrm{vib}}$, which is initially unknown. The lack of radio observations of these regions hinders an accurate determination of the $\mathrm{HCN}$ abundance. Hence, it is better to obtain the $\mathrm{H}^{13} \mathrm{CN}$ abundance by fitting the optically thin $\nu_{2}(\pi)-$ G.S. $\left(\sigma^{+}\right)$lines and using the derived ratio $\left[{ }^{12} \mathrm{C}\right] /\left[{ }^{13} \mathrm{C}\right] \simeq 41$ to determine the abundance of HCN. We found that the abundances that produce the best fits are

$$
x_{\mathrm{HCN}}(r)= \begin{cases}1.23 \times 10^{-5}, & \text { region } \mathrm{I}, \\ 4.5 \times 10^{-5}, & \text { region } \mathrm{II}, \\ 2.0 \times 10^{-5}, & \text { region III, }\end{cases}
$$

which means that the column density is $1.6 \times 10^{19} \mathrm{~cm}^{-2}$, i.e., similar to that of $\mathrm{C}_{2} \mathrm{H}_{2}$. In fact, $\mathrm{HCN}$ seems to be more abundant than $\mathrm{C}_{2} \mathrm{H}_{2}$ in region $\mathrm{I}$, where the gas density is rather high. However, as in the $\mathrm{C}_{2} \mathrm{H}_{2}$ case, the inner abundance is poorly determined (see $\S 8$ ), and the middle and outer ones are compatible with those found in previous works (Keady \& Ridgway 1993; Cernicharo et al. 1996, 1999; Wiedemann et al. 1991; Dayal \& Bieging 1995; Lindqvist et al. 2000). The abundance of HCN between regions I and II increases by a factor of 3.7, while for $\mathrm{C}_{2} \mathrm{H}_{2}$ the increase is by a factor of 10 , incompatible in this case with the predictions of chemical LTE models (M. Agúndez 2006, private communication) and non-LTE stellar pulsating ones (Cherchneff 2006), where a diminishing in the HCN abundance with growing radii is found (see $\S 8$ for a discussion on this topic). The decrease by a factor of $\simeq 2$ in the abundance between regions II and III seems to be real (see Table 7).

\section{SENSITIVITY TO DIVERSE PARAMETERS AND DISCUSSION}

To obtain complete information about the CSE, it is necessary to study the behavior of the model while varying the parameters and their uncertainties. For this purpose, we have selected lines of all the molecules studied in the present work. Each line is radiatively active over different regions of the CSE. For example, the $\mathrm{H}^{13} \mathrm{CCH}$ line $2 \nu_{5}(\delta) \leftrightarrow \nu_{5}(\pi) R_{e}(7)$ extends until $22 R_{*}$ and has an optical depth of $8.50 \times 10^{-2}$ in region I, 1.41 in region II, and $9.94 \times 10^{-3}$ in region III. On the other hand, the $\mathrm{C}_{2} \mathrm{H}_{2}$ line $\nu_{5}\left(\pi_{u}\right) \leftrightarrow$ G.S. $\left(\sigma_{g}^{+}\right) R_{e}(30)$ extends until $27.5 R_{*}$ and has an optical depth of 3.39 in region I, 39.0 in region II, and 1.35 in region III. The first line is useful to study region II while the second is useful for region III because they are optically thin. Table 7 shows the calculated errors and the lines used for this purpose. The chosen lines appear to be the best for a determination of the errors in the physical parameters. The velocity gradients introduce different spatial contributions to the line profiles. Hence, the impact of each region of the CSE becomes measurable through the analysis of these lines. Moreover, a line that is globally optically thick can also carry information on specific regions of the envelope. However, there are some parameters such as $T_{d 1}$ or $R_{d 1}$ whose errors must be calculated by fitting only the continuum because the changes they produce on the lines, when varied, can be overcome by modifying other parameters, e.g., vibrational temperatures.

The results obtained in this paper have been based on several hypotheses, most of them supported by observational data. However, the assumption for which the observations of IRC +10216 by ISO SWS and IRTF TEXES are compatible (see $\S 4$ ) needs discussion. As we pointed out in $\S 2$, the difference in the IR phase between both observations is $\Delta \phi_{\mathrm{IR}} \simeq 0.3$. Hence, the physical properties in the innermost CSE are different for observations of gas (IRTF TEXES) and dust (ISO SWS). Each pulsation of the star is followed by an increment in the emitted radiation, and consequently $R_{d 1}$ and $T_{d 1}$ are magnified because of the new input of optical and IR photons from the star and the ejected gas. Nevertheless, the large optical depth of dust shells near $R_{d 1}$ diminishes the number of short-wavelength photons from the stellar surface as the radius increases. The rest of the high-frequency stellar emission is largely diluted and does not significantly affect the middle and outer envelope. The result is that only the inner shells of the dusty CSE are significantly heated. The dust grains near $R_{d 1}$ reemit the absorbed energy at longer wavelengths, increasing the observed NIR intensity of the continuum. Since FIR and radio emission from the source arises almost entirely from the outer dust shells (the blackbody emission at $T_{\mathrm{bb}}=150 \mathrm{~K}$ peaks at $19.3 \mu \mathrm{m}$ and the dust shell at that temperature at $\simeq 450 R_{*}$ ), stellar pulsation has little effect on the long-wavelength range of the continuum. However, it does affect the MIR contribution (Monnier et al. 1998). By modifying $T_{d 1}$ from 850 to $950 \mathrm{~K}$ and the exponent $\alpha_{d}$ from 0.39 to 0.42 to model the increment of $T_{d}$ in the inner dusty CSE during a stellar pulsation, the continuum changes less than $15 \%$ over the frequency range considered in this paper. Any variations introduced in the line shapes can be overcome by changing the $T_{\mathrm{ev}}$ profiles. Despite these changes, the rough behavior of $T_{\mathrm{ev}}$ remains. The abundance profile does not seem to be affected by these modifications of $T_{d}$. The increase in the abundance detected between regions I and II seems to be real and not an effect of an incompatibility between the ISO SWS and IRTF TEXES observations.

The expansion velocity in region I derived by us is larger than that suggested by Keady et al. (1988) and Keady \& Ridgway (1993) by a factor of 1.75 . The main effect produced by this difference on the P Cygni profiles is found at the red wing of the line emission, at positive velocities. Increasing the expansion velocity of the gas in region I expands the red wing of P Cygni profiles. A reduction to $3 \mathrm{~km} \mathrm{~s}^{-1}$ does not improve the fits. Expansion velocities larger than $5 \mathrm{~km} \mathrm{~s}^{-1}$ seem to give better results but are improbable. The synthetic profiles with $5 \mathrm{~km} \mathrm{~s}^{-1}$ fit the observed lines quite well, although a slight lack of emission at velocities near terminal in $\mathrm{C}_{2} \mathrm{H}_{2} \nu\left(\pi_{u}\right)-$ G.S. $\left(\sigma_{g}^{+}\right) R_{e}$ and $\mathrm{HCN}$ $\nu_{2}(\pi)-$ G.S. $\left(\sigma^{+}\right) R_{e}$ lines can be seen. 
TABLE 7

ERRORS

\begin{tabular}{|c|c|c|c|c|}
\hline Abundance & Region & Value & Parameter & Value \\
\hline \multirow[t]{3}{*}{ 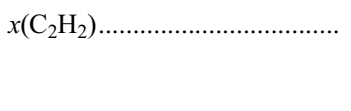 } & $\mathrm{I}$ & $8_{-4}^{+6} \times 10^{-6 a}$ & $R_{d 1}\left(R_{*}\right) \ldots \ldots \ldots \ldots \ldots \ldots$ & $5.2_{-0.5}^{+0.6 \mathrm{~b}}$ \\
\hline & II & $8.0_{-1.1}^{+1.2} \times 10^{-5 \mathrm{a}}$ & 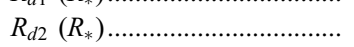 & $21 \pm 3^{\mathrm{c}}$ \\
\hline & III & $8.0_{-2.3}^{+4.0} \times 10^{-5 \mathrm{c}}$ & $T_{K}\left(R_{d 1}\right)(\mathrm{K})$ & $900_{-200}^{+300 \mathrm{~d}}$ \\
\hline \multirow[t]{3}{*}{$x\left(\mathrm{H}^{13} \mathrm{CCH}\right)$} & I & $3.7_{-1.5}^{+1.6} \times 10^{-7 \mathrm{e}}$ & $T_{K}\left(R_{d 2}\right)(\mathrm{K}) \ldots \ldots \ldots \ldots \ldots \ldots$ & $400_{-25}^{+27 \mathrm{c}}$ \\
\hline & II & $3.9_{-0.4}^{+0.5} \times 10^{-6 \mathrm{e}}$ & $\alpha_{\mathrm{I}}$ & $0.58 \pm 0.16^{\mathrm{d}}$ \\
\hline & III & $3.9_{-0.9}^{+1.0} \times 10^{-6 \mathrm{f}}$ & $\alpha_{\mathrm{II}} \ldots \ldots \ldots \ldots \ldots \ldots \ldots$ & $0.58_{-0.04}^{+0.05 \mathrm{c}}$ \\
\hline \multirow[t]{3}{*}{$x(\mathrm{HCN})$} & I & $(1.2 \pm 0.4) \times 10^{-5 \mathrm{~g}}$ & $T_{\mathrm{vib}}\left(\nu_{5} R_{e}, R_{d 1}\right)(\mathrm{K}) \ldots \ldots \ldots \ldots \ldots$ & $175_{-20}^{+2.04}$ \\
\hline & II & $4.5_{-0.6}^{+0.7} \times 10^{-5 \mathrm{~g}}$ & $T_{\mathrm{vib}}\left(\nu_{5} R_{e}, R_{d 2}\right)(\mathrm{K}) \ldots \ldots \ldots \ldots \ldots$ & $82_{-9}^{+10 \mathrm{c}}$ \\
\hline & III & $2.0_{-0.6}^{+0.8} \times 10^{-5 \mathrm{~h}}$ & 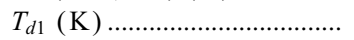 & $850 \pm 50^{\mathrm{b}}$ \\
\hline \multirow[t]{5}{*}{$x\left(\mathrm{H}^{13} \mathrm{CN}\right)$} & I & $3.0_{-0.9}^{+1.0} \times 10^{-7 \mathrm{~d}}$ & 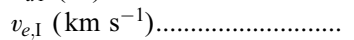 & $5.0_{-0.9}^{+1.6 \mathrm{~d}}$ \\
\hline & II & $1.10_{-0.16}^{+0.18} \times 10^{-6 \mathrm{~d}}$ & $v_{e, \mathrm{II}}\left(\mathrm{km} \mathrm{s}^{-1}\right) \ldots \ldots \ldots \ldots \ldots \ldots \ldots$ & $11.0_{-1.4}^{+1.5 \mathrm{i}}$ \\
\hline & III & $5.0_{-1.5}^{+2.0} \times 10^{-7 \mathrm{~g}}$ & $v_{e, \mathrm{III}}\left(\mathrm{km} \mathrm{s}^{-1}\right) \ldots \ldots \ldots \ldots \ldots \ldots \ldots$ & $14.5 \pm 0.4^{\mathrm{i}}$ \\
\hline & & & 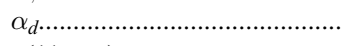 & $0.39_{-0.06}^{+0.08 \mathrm{~b}}$ \\
\hline & & & $\tau(11 \mu \mathrm{m})$ & $0.70_{-0.11}^{+0.13 \mathrm{~b}}$ \\
\hline
\end{tabular}

Notes.-The parameters derived by the model with their errors, estimated through a sensitivity study. All the errors translate into an interval of the normalized flux with a maximum width of $20 \%$, containing the best fit. The uncertainty of a given parameter has been calculated by comparing the synthetic spectrum with the observed one and by forcing the former to be within that interval, while the rest of the parameters are modified until getting the maximum/minimum value for the considered one (see $\S 3.2$ for more information about the method). Some of the values in the upper table could be slightly different from those appearing along the text, since the latter produce the best fits and the former produce just acceptable fits and are complemented with the uncertainties of the parameters. The $x$-values are the abundances in the three regions of the envelope. $R_{d 1}$ and $R_{d 2}$ are the positions of the inner and outer dust formation shells, respectively. The kinetic temperature, $T_{K}$, has been evaluated at $R_{d 1}$ and $R_{d 2}$. Parameters $\alpha_{\mathrm{I}}$ and $\alpha_{\mathrm{II}}$ are the $T_{K}$ exponents in regions I and II, respectively. $T_{\mathrm{vib}}\left(\nu_{5} R_{e}, R_{d 1}\right)$ and $T_{\mathrm{vib}}\left(\nu_{5} R_{e}, R_{d 2}\right)$ are the vibrational temperatures of the transition $\nu_{5}-$ G.S. $R_{e}$ evaluated at $R_{d 1}$ and $R_{d 2}$, respectively (shown in the table as an example of the $T_{\text {vib }}$ uncertainties). $T_{d 1}$ is the dust temperature at $R_{d}$. The expansion velocity of the gas, $v_{e}$, is shown over the three regions of the CSE. Parameter $\alpha_{d}$ is the exponent of the dust temperature law. Finally, the last parameter is the dust optical depth, $\tau$, at $11 \mu \mathrm{m}$. A list of the lines used to calculate the error for each parameter is shown below. Each line is accompanied by the set of parameters $P=\left[r_{1 \%}\left(R_{*}\right), \tau_{\text {total }}, \tau_{\mathrm{I}}, \tau_{\mathrm{II}}, \tau_{\mathrm{III}}\right]$, where $r_{1 \%}$ is the minimal radius at which the opacity of the line is $1 \%$ of the maximum, $\tau_{\text {total }}$ is the total optical depth, and $\tau_{\mathrm{I}}, \tau_{\mathrm{II}}$, and $\tau_{\text {III }}$ are the optical depths in regions I, II, and III, respectively.

${ }^{\mathrm{a}} \mathrm{C}_{2} \mathrm{H}_{2} 2 \nu_{5}\left(\delta_{g}\right) \leftrightarrow \nu_{5}\left(\pi_{u}\right) R_{e}(30)$ with $P=(20.1,3.57,0.549,2.83,0.00664)$.

b The errors have been calculated by fitting the continuum with different values of the parameter.

${ }^{\mathrm{c}} \mathrm{C}_{2} \mathrm{H}_{2} \nu_{5}\left(\pi_{u}\right) \leftrightarrow$ G.S. $\left(\sigma_{g}^{+}\right) R_{e}(30)$ with $P=(28.4,42.0,1.83,37.2,0.988)$.

${ }^{\mathrm{d}} \mathrm{H}^{13} \mathrm{CN} \nu_{2}(\pi) \leftrightarrow$ G.S. $\left(\sigma^{+}\right) R_{e}(14)$ with $P=(46.4,3.14,0.132,2.64,0.234)$.

${ }^{\mathrm{e}} \mathrm{H}^{13} \mathrm{CCH} 2 \nu_{5}(\delta) \leftrightarrow \nu_{5}(\pi) R_{e}(7)$ with $P=(22.6,1.47,0.0492,1.38,0.00660)$.

${ }^{\mathrm{f}} \mathrm{H}^{13} \mathrm{CCH} \nu_{5}(\pi) \leftrightarrow$ G.S. $\left(\sigma^{+}\right) R_{e}(26)$ with $P=(37.8,2.98,0.0629,2.59,0.188)$.

$\mathrm{g}$ The absolute error of the abundance in the given region for the isotopolog considered has been calculated with the relative error for the other isotopolog. Taking the ratio $\left[{ }^{12} \mathrm{C}\right] /\left[{ }^{13} \mathrm{C}\right]$ as constant throughout the CSE, the relative errors of both isotopologs must be equal.

${ }^{\mathrm{h}} \mathrm{HCN} \nu_{2}(\pi) \leftrightarrow$ G.S. $\left(\sigma^{+}\right) R_{e}(22)$ with $P=(32.8,62.6,5.16,53.9,1.07)$.

${ }^{\mathrm{i}} \mathrm{C}_{2} \mathrm{H}_{2} \nu_{5}\left(\pi_{u}\right) \leftrightarrow$ G.S. $\left(\sigma_{g}^{+}\right) R_{e}(6)$ with $P=(206,241,1.60,103.0,127.4)$.

In order to detect any effect produced by the difference between the pulsation phase of the IRTF TEXES and ISO SWS observations, we have varied the parameters $R_{d 1}$ and $T_{d}$. They do not cause any modification of the width of the red wing. The fact that the width of the red part of the observed P Cygni profiles is larger than that of the synthetic ones, considering the accepted microturbulence, i.e., $\Delta v_{1}=5 \mathrm{~km} \mathrm{~s}^{-1}$, suggests either more emission from the outer CSE only at the back of the star or larger line widths in the innermost envelope. The former explanation is in disagreement with the spherical symmetry established by a large number of observations. The latter seems to be more realistic. A possible explanation could be an onion-structured region I with shells expanding at positive and negative velocities corresponding to expansion and collapse (Bowen 1988). However, we could suggest another scenario explaining large line widths in the innermost CSE where it would be a clumpy region. The clumps would be composed of hot gas and would move inward and outward at high velocities along different radial directions. Unfortunately, little information on the spatial distribution of these clumps in region I could be derived through the observed spectrum due to heavy dilution. Both scenarios have been invoked by Fonfría Expósito et al. (2006) in order to explain the observed SiS masers toward IRC +10216 , where each maser feature has widths of $\simeq 2-3 \mathrm{~km} \mathrm{~s}^{-1}$, providing valuable information about the physical conditions of the emitting regions, i.e., shells or clumps. Modeling these structures as in $\S 5.2$ with $\Delta v_{1}=30 \mathrm{~km} \mathrm{~s}^{-1}$ and $\ell=1.5 R_{*}$ produces synthetic profiles having an emission wing wider than with $\Delta v_{1}=5 \mathrm{~km} \mathrm{~s}^{-1}$. Related to the derived column densities for $\mathrm{C}_{2} \mathrm{H}_{2}$ and $\mathrm{HCN}$, they are beam averaged and thus their actual values for the clumps in region I could be much larger if we take into account dilution. More spatial resolution is needed to study the complex, small-scale structure of the innermost CSE.

The possible condensation of $\mathrm{C}_{2} \mathrm{H}_{2}$ (and isotopologs) onto the dust grains in the outer acceleration zone is somewhat constrained by our derived uncertainties in the abundances. In regions II and III, these allow for $20 \%-30 \%$ of the $\mathrm{C}_{2} \mathrm{H}_{2}$ to be deposited onto grains. We can estimate how much the dust grains should increase in radius from this deposition. Using the density of $\mathrm{C}_{2} \mathrm{H}_{2}$ and the density of dust grains at $R_{d 2}$ derived from the fits $(\simeq 1200$ and $\simeq 3 \times 10^{-4} \mathrm{~cm}^{-3}$, respectively), assuming that $30 \%$ of the $\mathrm{C}_{2} \mathrm{H}_{2}$ turns into solid state with density $\simeq 1 \mathrm{~g} \mathrm{~cm}^{-3}$ and that the dust grains are spheres of radius $0.05 \mu \mathrm{m}$, we calculate that $1.2 \times$ $10^{6}$ molecules of $\mathrm{C}_{2} \mathrm{H}_{2}$ condense onto a single dust grain. The contribution to the mass of the dust grain is $10 \%$, and the radius of the grain grows by $3.2 \%$. For a larger initial dust grain radius, the increase is even smaller. The small increase in the size of the dust grains suggests that the condensation of $\mathrm{C}_{2} \mathrm{H}_{2}$ onto the dust grains does not contribute significantly to the grain growth and 
so other carbon molecules are more likely to be responsible for it. However, it might be possible that $\mathrm{C}_{2} \mathrm{H}_{2}$ reacts with other molecules on the grain surface, giving rise to more complex organic molecules.

Regarding the decrease of the $\mathrm{HCN}$ abundance in $R_{d 2}$, it might be thought that $\mathrm{HCN}$ suffers a depletion onto dust grains. In that case, it would be reasonable to expect some emission/absorption in the observed continuum at wavelengths between 4 and $5 \mu \mathrm{m}$ due to the stretching mode of the group CN (see, e.g., Lacy et al. 1984; Pendleton et al. 1999). Nevertheless, no significant emission/ absorption can be seen in the ISO SWS spectrum of IRC +10216 at these wavelengths although it is possible that some $\mathrm{HCN}$ molecules condense onto dust grains. However, their contribution to the continuum could be masked by the $\mathrm{CO}$ vibrational band at $4.67 \mu \mathrm{m}$. Even in that case, we think that this deposition process does not account for the abundance decay observed at $R_{d 2}$, which could actually be due to chemical processes. The most important HCN decrease is produced by photodissociation in the external layers of the CSE (Agúndez \& Cernicharo 2006 and references therein).

\section{CONCLUSIONS}

IRC +10216 has been observed from 11 to $14 \mu \mathrm{m}$ with the high-resolution spectrograph TEXES at the $3 \mathrm{~m}$ IRTF. We complemented these data with information on the continuum observed with $I S O$ SWS. We have identified 462 rovibrational lines of $\mathrm{C}_{2} \mathrm{H}_{2}$ (involving vibrational levels up to $3 \nu_{5}$ at $\simeq 2185 \mathrm{~cm}^{-1}$ ), 95 of HCN (involving levels up to $2 \nu_{2}$ at $\simeq 1425 \mathrm{~cm}^{-1}$ ), 106 of $\mathrm{H}^{13} \mathrm{CCH}$ (involving levels up to $2 \nu_{5}$ at $\simeq 1454 \mathrm{~cm}^{-1}$ ), and 7 of $\mathrm{H}^{13} \mathrm{CN}$ (the fundamental band $\nu_{2}$ at $\simeq 707 \mathrm{~cm}^{-1}$ ). By means of a model of an AGB star developed by us, we have fitted over 300 lines. The results can be summarized as follows:

1. The geometrical structure and physical properties of dust and gas over the whole envelope are compatible in many cases with those already proposed. However, the values of certain parameters are quite different. In particular, we find the position of the outer acceleration zone $\left(\simeq 21 R_{*} \simeq 0.4^{\prime \prime}\right)$ to be farther out than found by previous work.

2. The abundances of $\mathrm{C}_{2} \mathrm{H}_{2}$ and $\mathrm{HCN}$ in the innermost CSE, region $\mathrm{I}\left(8 \times 10^{-6}\right.$ for $\mathrm{C}_{2} \mathrm{H}_{2}$ and $1.2 \times 10^{-5}$ for $\mathrm{HCN}$ with $\left[{ }^{13} \mathrm{C}\right] /\left[{ }^{12} \mathrm{C}\right] \simeq 41$ ), are lower than those over the outer envelope, regions II $\left(8.0 \times 10^{-5}\right.$ for $\mathrm{C}_{2} \mathrm{H}_{2}$ and $4.5 \times 10^{-5}$ for $\left.\mathrm{HCN}\right)$ and III $\left(8.0 \times 10^{-5}\right.$ for $\mathrm{C}_{2} \mathrm{H}_{2}$ and $2.0 \times 10^{-5}$ for HCN $)$. For HCN, the ratio of the abundance in region II to that in region I is only a factor of $\simeq 4$, but in the case of $\mathrm{C}_{2} \mathrm{H}_{2}$ it is a factor of $\simeq 10$. The latter result is in accordance with the increase predicted by the chemical models. Contrarily, the derived abundance of HCN grows as radius increases while the chemical models suggest a decay. In addition, the determined abundances in regions II and III allow the condensation of $\leq 20 \%-30 \%$ of molecules of $\mathrm{C}_{2} \mathrm{H}_{2}$ onto the dust grains. On the other hand, the decrease of the $\mathrm{HCN}$ abundance when reaching the outer dust formation zone is probably due to chemical processes.

3. The vibrational temperatures determined by fitting the lines suggest the existence of a complex pumping mechanism driven by NIR radiation $(3.5-7 \mu \mathrm{m})$ emitted by the star and the inner dusty CSE. High-energy vibrational levels play an important role in the non-LTE pumping of low- and mid-energy vibrational states throughout region II and the edge of region III through radiative cascades. Reliable modeling using spectroscopic methods of any molecular species in the innermost CSE requires an analysis of high-energy vibrational levels at temperatures as high as $\simeq 3000 \mathrm{~K}$.

4. Most of the rotational levels behave as expected: low- $J$ rotational levels can be considered to be in LTE, while high- $J$ ones are not thermalized. Interestingly, we have found it necessary to add ad hoc rotational temperatures to fit several rovibrational lines. These $T_{\text {rot }}$ are quite different from those of adjacent levels. The involved rovibrational transitions do not seem to be affected by instrumental or telluric effects so we assume these variations to be real and produced by molecular processes such as overlaps with lines of other molecular species.

Infrared spectroscopic data provide us with extensive information about physical conditions of warm sources with good spatial resolution and without resorting to interferometric methods. However, physical processes and the time-dependent chemistry in the innermost CSE remain unknown. Future high angular resolution observations with TEXES in the infrared domain will provide us with high-quality data to study the dynamics, chemistry, and physical conditions in the warm asymmetric innermost envelope. On the other hand, ALMA will supply us in several years with the interferometric observations needed to delve more deeply into the dynamics of the near environment of the central star and to further our understanding of dust formation and growth.

J. C. and J. P. F. would like to thank the Spanish Ministerio de Educación y Ciencia for funding support through grant ESP2004665, AYA2003-2785, and the "Comunidad de Madrid" government under PRICIT project S-0505/ESP-0237 (ASTROCAM). During this study, J. P. F. was supported by the CSIC and the "Fondo Social Europeo" under internship grant from the I3P Programme. This study is supported in part by the European Community's human potential Programme under contract MCRTNCT-2004-51230, "Molecular Universe". M. J. R. is supported by grant AST 03-07497. Development of TEXES was supported by grants from the NSF and USRA. Observations with TEXES were supported by NSF grant AST 02-05518 and AST 06-07312. M. J. R., J. H. L., and others want to thank IRTF, which is operated by the University of Hawaii under Cooperative Agreement NCC 5-538 with the National Aeronautics and Space Administration, Office of Space Science, Planetary Astronomy Program. We also thank J. R. Pardo, M. Taylor, and the referee for suggestions and valuable corrections to this manuscript, $\mathrm{M}$. Agúndez for the very interesting talks about chemistry in the inner envelope of IRC +10216 , and H. Mutschke for providing us with SiC opacities and his comments on dust properties.

\section{APPENDIX A}

\section{LINE FREQUENCIES}

The spectroscopic data for all the molecular species have been taken mainly from the HITRAN Database (Rothman et al. 2003). Further information came from data published by Herman et al. (2003), Kabbadj et al. (1991), and Di Lonardo et al. (1993, 2002) for $\mathrm{C}_{2} \mathrm{H}_{2}$ and its isotopologs, and from Maki et al. $(1996,2000)$ and Devi et al. (2005) for $\mathrm{HCN}$ and $\mathrm{H}^{13} \mathrm{CN}$. The spectroscopic data relative to the modeled lines can be found in Table 8 . 
TABLE 8

Spectroscopic Data of the Modeled Lines

\begin{tabular}{|c|c|c|c|c|c|c|c|}
\hline $\begin{array}{l}\text { Molecule } \\
\text { (1) }\end{array}$ & $\begin{array}{l}\text { Transition } \\
\text { (2) }\end{array}$ & $\begin{array}{l}\text { Frequency } \\
\left(\mathrm{cm}^{-1}\right) \\
\text { (3) }\end{array}$ & $\begin{array}{c}R^{2} \\
\left(10^{-2} \mathrm{D}^{2}\right) \\
(4)\end{array}$ & $\begin{array}{c}E_{l} \\
\left(\mathrm{~cm}^{-1}\right) \\
(5)\end{array}$ & $\begin{array}{l}S_{u l} \\
(6)\end{array}$ & $\begin{array}{l}g_{u} \\
(7)\end{array}$ & $\begin{array}{c}g_{l} \\
(8)\end{array}$ \\
\hline $\mathrm{HCN}$ & $2 \nu_{2}(\delta) \leftrightarrow \nu_{2}(\pi) R_{f}(3)$ & 726.423637 & 7.460 & 729.8054 & 37.500 & 9 & 7 \\
\hline $\mathrm{HCN}$ & $2 \nu_{2}\left(\sigma^{+}\right) \leftrightarrow \nu_{2}(\pi) R_{e}(8)$ & 726.700075 & 3.740 & 818.3829 & 40.000 & 19 & 17 \\
\hline $\mathrm{H}^{13} \mathrm{CCH}$ & $\nu_{5}(\pi) \leftrightarrow$ G.S. $\left(\sigma^{+}\right) R_{e}(1)$ & 732.820280 & 8.744 & 2.2969 & 15.000 & 5 & 3 \\
\hline $\mathrm{H}^{13} \mathrm{CCH}$ & $\nu_{4}+\nu_{5}\left(\sigma^{+}\right) \leftrightarrow \nu_{4}(\pi) R_{e}(7)$ & 734.177200 & 5.118 & 671.4353 & 35.000 & 17 & 15 \\
\hline $\mathrm{H}^{13} \mathrm{CCH}$ & $\nu_{5}(\pi) \leftrightarrow$ G.S. $\left(\sigma^{+}\right) R_{e}(2)$ & 735.115630 & 6.995 & 6.8907 & 20.000 & 7 & 5 \\
\hline $\mathrm{HCN}$ & $2 \nu_{2}(\delta) \leftrightarrow \nu_{2}(\pi) R_{f}(6)$ & 735.315339 & 7.480 & 774.3662 & 51.429 & 15 & 13 \\
\hline $\mathrm{C}_{2} \mathrm{H}_{2} \ldots \ldots \ldots \ldots \ldots \ldots \ldots$ & $\nu_{4}+\nu_{5}\left(\sigma_{u}^{+}\right) \leftrightarrow \nu_{4}\left(\pi_{g}\right) R(7)$ & 735.543410 & 5.118 & 677.5067 & 35.000 & 51 & 45 \\
\hline $\mathrm{HCN}$ & $\nu_{2}(\pi) \leftrightarrow$ G.S. $\left(\sigma^{+}\right) R_{e}(7)$ & 735.611573 & 3.740 & 82.7713 & 45.000 & 17 & 15 \\
\hline $\mathrm{H}^{13} \mathrm{CCH}$ & $\nu_{5}(\pi) \leftrightarrow$ G.S. $\left(\sigma^{+}\right) R_{e}(3)$ & 737.410380 & 6.247 & 13.7813 & 25.000 & 9 & 7 \\
\hline $\mathrm{C}_{2} \mathrm{H}_{2} \ldots \ldots \ldots \ldots$ & $\nu_{4}+\nu_{5}\left(\sigma_{u}^{+}\right) \leftrightarrow \nu_{4}\left(\pi_{g}\right) R_{e}(8)$ & 737.980170 & 5.176 & 696.3085 & 40.000 & 19 & 17 \\
\hline
\end{tabular}

Notes.-Spectroscopic data relative to the modeled lines. The transitions are labeled in cols. (1) and (2), while in cols. (3) - (8) are shown the frequency in $\mathrm{cm}^{-1}$, the square dipole moment in $10^{-2} \mathrm{D}^{2}$, the energy of the lower level in $\mathrm{cm}^{-1}$, the line strength of each transition, and the degeneracy of the upper and lower levels, respectively. See the text for details on the notation relative to the transitions. Table 8 is published in its entirety in the electronic edition of the Astrophysical Journal. A portion is shown here for guidance regarding its form and content.

The molecules studied in the present work, acetylene and hydrogen cyanide, are linear. Consequently, the notation we have used is the same for both species. The notation adopted to refer to the vibrational normal modes is $v_{i} \nu_{i}\left(\ell_{S}^{p}\right)$, where $i$ is the mode number $(i=1$, $2, \ldots, 3 N-5-d$, where $N$ is the number of atoms of the molecule and $d$ is the number of degenerate modes), $v_{i}$ is the vibrational quantum number related to the $i$ th normal mode, $\ell$ is a letter corresponding to the quantum number of the total vibrational angular momentum of the vibrational state $(\ell=0 \equiv \sigma, \ell=1 \equiv \pi, \ell=2 \equiv \delta$, etc.), $S$ is the symmetry of the rovibrational state with respect to the molecular midplane ( $S=g$ for gerade or even states and $S=u$ for ungerade or odd ones), and $p$ is the parity of the total molecular wave function $(+,-)$. If no parity indicator appears, the label refers to the + and - levels simultaneously. If no symmetry indicator is present, the molecule is not symmetric with respect to the molecular midplane, as is the case of $\mathrm{H}^{13} \mathrm{CCH}$ and $\mathrm{HCN}$. For the combination bands, $\ell, S$, and $p$ refer to the mixture of all vibrational modes that participate in the combined state. Combination bands are sometimes split in different vibrational levels having the same quantum number $\ell \neq 0$, each one with both parities + and - . In these cases, Roman numbers are added to label these states from higher to lower energies (see Fig. 10). In addition, the interaction between the vibrational and molecular angular momenta splits the rotational levels of vibrational states with $\ell \neq 0$ into two sublevels with opposite parities denoted $e$ and $f$. The energy gap between those sublevels for a given rotational state depends on $J$ and on the vibrational state. For low- $J$ levels it is between 200 and 300 times lower than the rotational constant for acetylene and hydrogen cyanide. The gaps are large enough to be observed in high-resolution spectra, as in our case. In the label for each transition, the parity $e$ or $f$ of the lower rovibrational level is included. Deriving the parity of the upper level can be done with the selection rules governing transitions in linear molecules:

$$
\begin{aligned}
& \Delta v_{i}= \pm 1, \\
& \Delta v_{i}= \pm 1, \pm 2, \pm 3, \ldots, \\
& \Delta \ell=0, \pm 1, \\
& \sigma^{+} \leftrightarrow \sigma^{-}, \\
& g \leftrightarrow u, \\
& g \leftrightarrow g, u \leftrightarrow u, \\
& \Delta J=0, \pm 1, \\
& e \leftrightarrow e, f \leftrightarrow f, \\
& e \leftrightarrow f,
\end{aligned}
$$$$
i=\text { bending modes, }
$$$$
i=\text { stretching modes, }
$$$$
\Delta \ell=0 \text { allowed only for } \ell_{\text {low }}=0,
$$

forbidden,

allowed $\left(\mathrm{C}_{2} \mathrm{H}_{2},{ }^{13} \mathrm{C}_{2} \mathrm{H}_{2}\right)$,

forbidden $\left(\mathrm{C}_{2} \mathrm{H}_{2},{ }^{13} \mathrm{C}_{2} \mathrm{H}_{2}\right)$,

$\Delta J=0$ forbidden for $J=0 \leftrightarrow J=0$, when $\Delta \ell=0$ and $\ell_{\text {low }}=0$, and

for parallel transitions; allowed otherwise,

allowed for $R$ - and $P$-branches; forbidden for $Q$-branch,

allowed for $Q$-branch; forbidden for $R$ - and $P$-branches,

where the transitions with $\Delta \ell=0$ are named parallel transitions (with the change in the dipole moment parallel to the molecular axis) and those with $\Delta \ell= \pm 1$ are named perpendicular transitions (the dipole moment changing in a perpendicular direction to the molecular axis).

The three most abundant isotopologs of acetylene are ${ }^{12} \mathrm{C}_{2} \mathrm{H}_{2}\left(\mathrm{C}_{2} \mathrm{H}_{2}\right), \mathrm{H}^{13} \mathrm{C}^{12} \mathrm{CH}\left(\mathrm{H}^{13} \mathrm{CCH}\right)$, and ${ }^{13} \mathrm{C}_{2} \mathrm{H}_{2}$. Acetylene is a linear molecule with five fundamental vibrational modes (see Fig. 10). The bending modes $\nu_{4}$ and $\nu_{5}$ are doubly degenerate to a first approximation with energies of 612.871 and $730.332 \mathrm{~cm}^{-1}$, respectively (Herman et al. 2003). The $\nu_{4}$ mode is infrared inactive for $\mathrm{C}_{2} \mathrm{H}_{2}$ and ${ }^{13} \mathrm{C}_{2} \mathrm{H}_{2}$, but active for $\mathrm{H}^{13} \mathrm{CCH}$. The $\nu_{1}, \nu_{2}$, and $\nu_{3}$ vibrational modes correspond to the different stretching modes of acetylene and have energies of $3372.849,1974.316$, and $3294.839 \mathrm{~cm}^{-1}$, respectively, for $\mathrm{C}_{2} \mathrm{H}_{2}$ and differ slightly for the isotopologs (Herman et al. 2003). Both $\mathrm{C}_{2} \mathrm{H}_{2}$ and ${ }^{13} \mathrm{C}_{2} \mathrm{H}_{2}$ are symmetric and show a degeneracy, $g_{s}$, in the rotational levels due to nuclear spin statistics. The para levels $(J=$ even $)$ have $g_{s}\left(\mathrm{C}_{2} \mathrm{H}_{2}\right)=1$ and $g_{s}\left({ }^{13} \mathrm{C}_{2} \mathrm{H}_{2}\right)=6$, while the ortho levels $(J=$ odd $)$ have $g_{s}\left(\mathrm{C}_{2} \mathrm{H}_{2}\right)=3$ and $g_{s}\left({ }^{13} \mathrm{C}_{2} \mathrm{H}_{2}\right)=10$. 
TABLE 9

Dipole Moments for $\mathrm{C}_{2} \mathrm{H}_{2}$ and HCN Vibrational Transitions

\begin{tabular}{|c|c|c|}
\hline Transition & $g_{v}$ & $\begin{array}{c}\left|R_{0}\right|^{2} \\
\left(\times 10^{-2} \mathrm{D}^{2}\right)\end{array}$ \\
\hline \multicolumn{3}{|c|}{$\mathrm{C}_{2} \mathrm{H}_{2}$} \\
\hline 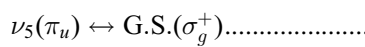 & 1 & $9.81 \pm 0.11^{\mathrm{a}}$ \\
\hline$\nu_{4}+\nu_{5}\left(\sigma_{u}^{+}\right) \leftrightarrow \nu_{4}\left(\pi_{g}\right) \ldots \ldots \ldots \ldots \ldots \ldots$ & 1 & $4.904 \pm 0.063^{\mathrm{a}}$ \\
\hline$\nu_{4}+\nu_{5}\left(\sigma_{u}^{-}\right) \leftrightarrow \nu_{4}\left(\pi_{g}\right) \ldots \ldots \ldots \ldots \ldots$ & 1 & $5.006 \pm 0.062^{\mathrm{a}}$ \\
\hline$\nu_{4}+\nu_{5}(\delta) \leftrightarrow \nu_{4}\left(\pi_{g}\right) \ldots \ldots \ldots \ldots \ldots$ & 2 & $9.599 \pm 0.084^{\mathrm{a}}$ \\
\hline 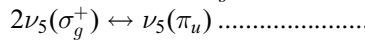 & 1 & $9.79 \pm 0.19^{\mathrm{a}}$ \\
\hline 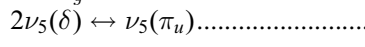 & 2 & $18.86 \pm 0.14^{\mathrm{a}}$ \\
\hline \multicolumn{3}{|c|}{$\mathrm{HCN}$} \\
\hline$\nu_{2}(\pi) \leftrightarrow$ G.S. $\left(\sigma^{+}\right)$ & 1 & $3.8925 \pm 0.0016^{\mathrm{b}}$ \\
\hline $2 \nu_{2}\left(\sigma^{+}\right) \leftrightarrow \nu_{2}(\pi)$ & 1 & $3.39^{\mathrm{c}}$ \\
\hline $2 \nu_{2}(\delta) \leftrightarrow \nu_{2}(\pi)$ & 2 & $7.14^{\mathrm{c}}$ \\
\hline
\end{tabular}

Note.-See the text for a definition of $g_{v}$ and the rovibrational selection rules.

a Jacquemart et al. (2001).

${ }^{b}$ Devi et al. (2005).

c The vibrational dipole moment has been obtained from fits to the available data in the HITRAN Database (http://cfa-www.harvard.edu/hitran/). Devi et al. (2005) established that the values of this database for the $\nu_{2} \leftrightarrow$ G.S. transition are lower than they should be. Hence, we could expect the same for the other transitions. Therefore, we use the same values for $2 \nu_{2}\left(\sigma^{+}\right) \leftrightarrow \nu_{2}(\pi)$ and $2 \nu_{2}(\delta) \leftrightarrow \nu_{2}(\pi)$ rather than $\nu_{2}(\pi) \leftrightarrow$ G.S. $\left(\sigma^{+}\right)$(Devi et al. 2005) as an approximation.

These two isotopologs have a null permanent dipole moment due to symmetry with respect to the molecular midplane. Therefore, they do not have purely rotational transitions. However, $\mathrm{H}^{13} \mathrm{CCH}$ is not symmetric with respect the molecular midplane and has a very small dipole moment along the molecular axis. The bands of $\mathrm{C}_{2} \mathrm{H}_{2}$ and $\mathrm{H}^{13} \mathrm{CCH}$ identified are shown in Table 2.

$\mathrm{HCN}$ (and $\mathrm{H}^{13} \mathrm{CN}$ ) has a permanent dipole moment along the molecular axis allowing purely rotational radiative transitions. HCN has three vibrational modes: $\nu_{2}$ is a doubly degenerate bending mode with an energy of $713.461 \mathrm{~cm}^{-1}$, and the other two, $\nu_{1}$ and $\nu_{3}$, are stretching modes with energies of 3311.480 and $2096.846 \mathrm{~cm}^{-1}$, respectively (Maki et al. 2000). The vibrational energy pattern for $\mathrm{HCN}$ and $\mathrm{H}^{13} \mathrm{CN}$ is shown in Figure 15. The detected bands are given in Table 2.

\section{APPENDIX B}

\section{LINE INTENSITIES}

The line intensity as a function of temperature is given by (Jacquemart et al. 2001)

$$
S(T)=\frac{1}{4 \pi \varepsilon_{0}} \frac{8 \pi^{3}}{3 h c} \frac{g_{\mathrm{low}}^{S} \nu_{0}}{Z(T)} \frac{1}{g_{v}}|R|^{2} L(J, \ell) e^{-h c E_{\mathrm{low}} / k_{\mathrm{B}} T}\left(1-e^{-h c \nu_{0} / k_{\mathrm{B}} T}\right),
$$

where the dipole moment squared, $|R|^{2}$, is expressed in $\mathrm{D}^{2}, \nu_{0}$ is the center of the line in $\mathrm{cm}^{-1}, E_{\mathrm{low}}$ is the energy of the lower level in $\mathrm{cm}^{-1}$, $g_{\text {low }}^{S}$ is the spin degeneracy of the lower level, $L(J, \ell)$ is the rotational line strength (see below), and $Z(T)$ is the partition function. Here $g_{v}=2$ when the upper and lower vibrational levels involved in a rovibrational transition present $\ell$-type doubling, i.e., $\ell_{\text {up }}, \ell_{\text {low }} \neq 0$; otherwise, $g_{v}=1$. In the case of $g_{v}=2$, the given value of $|R|^{2}$ considers the transitions having parities $e$ and $f$ at the same time.

The line strength of a rovibrational transition, $L(J, \ell)$, for a linear molecule is given by (Rothman et al. 1992; Herzberg 1989a)

$$
L(J, \ell)= \begin{cases}(J+1+\ell)(J+1-\ell) /(J+1), & R \text {-branch, } \Delta \ell=0, \\ (2 J+1) \ell^{2} / J(J+1), & Q \text {-branch, } \Delta \ell=0, \\ (J+\ell)(J-\ell) / J, & P \text {-branch, } \Delta \ell=0, \\ (J+2+\ell \Delta \ell)(J+1+\ell \Delta \ell) / 2(J+1), & R \text {-branch, } \Delta \ell= \pm 1, \\ (J+1+\ell \Delta \ell)(J-\ell \Delta \ell)(2 J+1) / 2 J(J+1), & Q \text {-branch, } \Delta \ell= \pm 1, \\ (J-1-\ell \Delta \ell)(J-\ell \Delta \ell) / 2 J, & P \text {-branch, } \Delta \ell= \pm 1 .\end{cases}
$$

The dipole moments used to calculate the spectrum of $\mathrm{C}_{2} \mathrm{H}_{2}$ and the $\nu_{5}$ rovibrational transitions of $\mathrm{H}^{13} \mathrm{CCH}$ have been obtained from the HITRAN Database, while the hot bands of $\mathrm{H}^{13} \mathrm{CCH}$ have been assumed to be identical to those of $\mathrm{C}_{2} \mathrm{H}_{2}$. The corresponding values for the $\nu_{2}$ transition of HCN have been taken from Devi et al. (2005). The adopted dipole moments for HCN $\Delta \nu_{2}=1$ hot bands and for $\mathrm{H}^{13} \mathrm{CN} \nu_{2}$ transitions are similar to that of the $\nu_{2}$ transition of HCN. All dipole moments are shown in Table 9. 
The partition function has been calculated by directly summing rovibrational levels under these conditions:

1. For each vibrational state, at least all the rotational levels with $J \leq J_{\max }=65$ are summed.

2. The relative contribution to the rotational partition function of the last rotational level of each vibrational state must be less than $10^{-3}$, which may require a sum over rotational levels having $J>J_{\max }=65$.

3. The highest considered vibrational state, $v_{\max }$, must be higher than that of the upper rovibrational level involved in the considered transition.

4. The relative contribution to the molecular partition function of $v_{\max }$ (including the rotational partition function) must be less than $10^{-3}$, implying the possibility of summing vibrational levels with $v>v_{\max }$.

For the high rotational temperatures prevailing near the stellar photosphere, condition 2 implies that the highest rotational level needed to be included for $\mathrm{C}_{2} \mathrm{H}_{2}$ in the models is in the range $J=116-130$. Conditions 3 and 4 lead to $v_{\max }=110$. On the other hand, for low and intermediate $T_{K}$, the highest rotational level included to fulfill condition 2 is well below $J_{\max }=65$, e.g., for $T_{\text {rot }} \simeq 400 \mathrm{~K}$ the error on the partition function will be $10^{-3}$ for $J \simeq 32$, although we have to sum up to $J_{\max }=65$ to fulfill condition 1 , and $v_{\max }=9$ (conditions 3 and 4).

Agúndez, M., \& Cernicharo, J. 2006, ApJ, 650, 374

Bergeat, J., Knapik, A., \& Rutily, B. 2001, A\&A, 369, 178

Betz, A. L. 1981, ApJ, 244, L103

Bowen, G. H. 1988, ApJ, 329, 299

Cernicharo, J., \& Guélin, M. 1987, A\&A, 183, L10 1996, A\&A, 309, L27

Cernicharo, J., Guélin, M., \& Kahane, C. 2000, A\&AS, 142, 181

Cernicharo, J., Guélin, M., \& Pardo, J. R. 2004, ApJ, 615, L145

Cernicharo, J., et al. 1996, A\&A, 315, L201 1999, ApJ, 526, L41

Cherchneff, I. 2006, A\&A, 456, 1001

Cherchneff, I., Barker, J. R., \& Tielens, A. G. G. M. 1992, ApJ, 401, 269

Cox, A. N., ed. 2000, Allen's Astrophysical Quantities (4th ed.; New York: AIP)

Crosas, M., \& Menten, K. M. 1997, ApJ, 483, 913

Dayal, A., \& Bieging, J. H. 1993, ApJ, 407, L37 1995, ApJ, 439, 996

Devi, V. M., et al. 2005, J. Mol. Spectrosc., 231, 66

Di Lonardo, G., et al. 1993, J. Mol. Spectrosc., 161, 466 2002, J. Mol. Spectrosc., 213, 57

Doty, S. D., \& Leung, C. M. 1997, MNRAS, 286, 1003

Dyck, H. M., et al. 1991, AJ, 102, 200

Fonfría Expósito, J. P., Agúndez, M., Tercero, B., Pardo, J. R., \& Cernicharo, J. 2006, ApJ, 646, L127

Gilman, R. C. 1972, ApJ, 178, 423

González-Alfonso, E., \& Cernicharo, J. 1997, A\&A, 322, 938

Groenewegen, M. A. T. 1997, A\&A, 317, 503

Guélin, M., et al. 1997, A\&A, 317, L1

Herbig, G. H., \& Zappala, R. R. 1970, ApJ, 162, L15

Herman, M., Campargue, A., Idrissi, M. I., \& Vander Auwera, J. 2003, J. Phys. Chem. Ref. Data, 32, 921

Herzberg, G. 1989a, Molecular Spectra and Molecular Structure, Vol. I: Spectra of Diatomic Molecules (Melbourne: Krieger)

. 1989b, Molecular Spectra and Molecular Structure, Vol. II: Infrared and Raman Spectra of Polyatomic Molecules (Melbourne: Krieger)

Hoyle, F., \& Wickramasinghe, N. C. 1991, The Theory of Cosmic Grains (Dordrecht: Kluwer)

Huggins, P. J., \& Healy, A. P. 1986, ApJ, 304, 418

Ivezić, Ž., \& Elitzur, M. 1996, MNRAS, 279, 1019

Jacquemart, D., et al. 2001, J. Quant. Spectrosc. Radiat. Transfer, 69, 81

Jones, T. J., et al. 1990, ApJS, 74, 785

Justtanont, K., et al. 2005, A\&A, 439, 627

Kabbadj, Y., et al. 1991, J. Mol. Spectrosc., 150, 535

Keady, J. J., Hall, D. N. B., \& Ridgway, S. T. 1988, ApJ, 326, 832

\section{REFERENCES}

Keady, J. J., \& Ridgway, S. T. 1993, ApJ, 406, 199

Knapp, G. R., \& Morris, M. 1985, ApJ, 292, 640

Kwok, S. 1975, ApJ, 198, 583

Lacy, J. H., et al. 1984, ApJ, 276, 533 2002, PASP, 114, 153

Lafont, S., Lucas, R., \& Omont, A. 1982, A\&A, 106, 201

Lindqvist, M., et al. 2000, A\&A, 361, 1036

Loup, C., et al. 1993, A\&AS, 99, 291

Lucas, R., et al. 1995, Ap\&SS, 224, 293

Maki, A., et al. 1996, J. Mol. Spectrosc., 180, 323

2000, J. Mol. Spectrosc., 202, 67

Mandin, J.-Y., et al. 2005, J. Quant. Spectrosc. Radiat. Transfer, 92, 239

Mauron, N., \& Huggins, P. J. 1999, A\&A, 349, 203 2000, A\&A, 359, 707

Men'shchikov, A. B., Hofmann, K.-H., \& Weigelt, G. 2002, A\&A, 392, 921

Men'shchikov, A. B., et al. 2001, A\&A, 368, 497

Monnier, J. D., Geballe, T. R., \& Danchi, W. C. 1998, ApJ, 502, 833

Monnier, J. D., et al. 2000, ApJ, 543, 861

Morris, M., et al. 1975, ApJ, 199, L47

Murakawa, K., et al. 2002, A\&A, 395, L9

Mutschke, H., et al. 1999, A\&A, 345, 187

Neugebauer, G., \& Leighton, R. B. 1969, Two Micron Sky Survey-a Preliminary Catalog (NASA SP-3047; Washington, DC: GPO)

Pendleton, Y. J., Tielens, A. G. G. M., \& Tokunaga, A. T. 1999, ApJ, 513, 294

Pijpers, F. P., \& Habing, H. J. 1989, A\&A, 215, 334

Pijpers, F. P., \& Hearn, A. G. 1989, A\&A, 209, 198

Ridgway, S. T., \& Keady, J. J. 1988, ApJ, 326, 843

Rothman, L. S., Hawkins, R. L., Wattson, R. B., \& Gamache, R. R. 1992, J. Quant. Spectrosc. Radiat. Transfer, 48, 537

Rothman, L. S., et al. 2003, J. Quant. Spectrosc. Radiat. Transfer, 82, 5

Rouleau, F., \& Martin, P. G. 1991, ApJ, 377, 526

Schilke, P., \& Menten, K. M. 2003, ApJ, 583, 446

Schöier, F. L., \& Olofsson, H. 2001, A\&A, 368, 969

Schöier, F. L., et al. 2006, ApJ, 649, 965

Skinner, C. J., Justtanont, K., Tielens, A. G. G. M., Betz, A. L., Boreiko, R. T., \& Baas, F. 1999, MNRAS, 302, 293

Tejero, J., \& Cernicharo, J. 1991, Modelos de Equilibrio Termodinámico Aplicados a Envolturas Circunestelares de Estrellas Evolucionadas (Madrid: IGN)

Tuthill, P. G., Monnier, J. D., \& Danchi, W. C. 2005, ApJ, 624, 352

Weigelt, G., et al. 1998, A\&A, 333, L51 2002, A\&A, 392, 131

Wiedemann, G. R., Deming, D., Jennings, D. E., Hinkle, K. H., \& Keady, J. J. 1991, ApJ, 382, 321 